\title{
Variability in the Glycosylation Patterns of gp120 Proteins from Different Human Immunodeficiency Virus Type 1 Isolates Expressed in Different Host Cells
}

\author{
Jingfu Zhao ${ }^{\ddagger 1}$, Ehwang Song ${ }^{\ddagger 1}$, Yifan Huang ${ }^{1}$, Aiying $\mathrm{Yu}^{1}$ and Yehia Mechref ${ }^{1 *}$ \\ ${ }^{1}$ Department of Chemistry and Biochemistry, Texas Tech University, Lubbock, TX 79409
}

*Corresponding author

Email: yehia.mechref@ttu.edu

Tel: 806-742-3059

Fax: 806-742-1289 
$¥$ Both authors contributed equally

\section{Table of Contents}

Table S1. List of identified glycopeptides associated with Ba-L gp120.

Table S2. List of identified glycopeptides associated with IIIB gp120.

Table S3. List of identified glycopeptides associated with 96ZM651 gp120.

Table S4. List of identified glycopeptides associated with 93TH975 gp120.

Table S5. Sequence similarity of different clade B and clade C gp120s from previous studies compared to those from this study.

Table S6. Comparisons of identified glycoforms from different clade B and clade C gp120s expressed in 293 or $293 \mathrm{~T}$ cells.

Table S7. Comparisons of identified glycoforms from different clade B and clade C gp120s expressed in 293/293T cells and CHO cells.

Figure S1. Venn plots of glycoforms detected at (A) N88, (B) N156-N160, (C) N332and (D) N234. On site N88, Ba-L and 96ZM651 shares 25 common structures. In which, 15 out of 25 structures were also detected in IIIB, while no glycans were detected in all 4 samples. On site N156-N160, the number of common structures detected in Ba-L, IIIB and 96ZM651 decreased to 8. Meanwhile 33 unique structures were identified from IIIB sample. Meanwhile, no common structure between the 4 samples were detected on site N332. Ba-L, in this case, has the most unique structures. On N234 site, all glycoforms detected from Ba-L and 93TH975 were also observed in the other 2 samples. On the contrary to N332, IIIB has the more unique glycans than the other 3 samples.

Figure S2. Types of glycoforms identified from the samples. The glycans were classified into 6 categories: high mannose, complex/hybrid without fucose or sialic acid, fucosylated but not sialylated, sialylated but not fucosylated, fucosylated and sialylated and N-glycans smaller than the pentasaccharide core.

Figure S3. Direct comparisons of glycoforms at N156-N160 glycosylation sites with previously published studies. The comparisons of total identified glycoforms (A), common glycoforms from the previous studies and this study (B), unique glycoforms in previously published studies compared to this study (C), and unique glycoforms from this study compared to previously published studies (D).

Figure S4. Direct comparisons of glycoforms at N332 glycosylation site with previously published studies. The comparisons of total identified glycoforms (A), common glycoforms from the previous studies and this study (B), unique glycoforms in previously published studies compared to this study (C), and unique glycoforms from this study compared to previously published studies (D). 
Zhao et al. Table S1

Table S1. List of identified glycopeptides associated with Ba-L gp120 .

\begin{tabular}{|c|c|c|c|c|c|c|}
\hline Domain & Peptide sequence & $\begin{array}{c}\text { Charge } \\
\text { state }\end{array}$ & $\begin{array}{c}\text { Theoretical } \\
m / z\end{array}$ & $\begin{array}{c}\text { Observed } \\
m / z\end{array}$ & $\begin{array}{c}\text { Mass } \\
\text { accurac } \\
\text { y (ppm) }\end{array}$ & Glycoform \\
\hline \multirow{39}{*}{$\mathrm{C} 1$} & \multirow{34}{*}{ LKNVTE } & 2 & 1053.9565 & 1053.9531 & -3.2 & HexNAc3Hex4Fuc1 \\
\hline & & 2 & 1074.4698 & 1074.4710 & 1.1 & HexNAc4Hex3Fuc1 \\
\hline & & 2 & 1155.4962 & 1155.4932 & -2.6 & HexNAc4Hex4Fuc1 \\
\hline & & 3 & 784.3421 & 784.3398 & -2.9 & HexNAc5Hex3Fuc1 \\
\hline & & 3 & 824.6842 & 824.6839 & -0.4 & HexNAc4Hex5Fuc1 \\
\hline & & 3 & 838.3597 & 838.3584 & -1.6 & HexNAc5Hex4Fuc1 \\
\hline & & 3 & 852.0352 & 852.0334 & -2.1 & HexNAc6Hex3Fuc1 \\
\hline & & 3 & 892.3773 & 892.3768 & -0.6 & HexNAc5Hex5Fuc1 \\
\hline & & 3 & 906.0528 & 906.0508 & -2.2 & HexNAc6Hex4Fuc1 \\
\hline & & 3 & 960.0704 & 960.0686 & -1.9 & HexNAc6Hex5Fuc1 \\
\hline & & 3 & 1014.0880 & 1014.0882 & 0.2 & HexNAc6Hex6Fuc1 \\
\hline & & 3 & 1062.7740 & 1062.7709 & -2.9 & HexNAc6Hex6Fuc2 \\
\hline & & 3 & 1068.1056 & 1068.1064 & 0.7 & HexNAc6Hex7Fuc1 \\
\hline & & 3 & 1081.7812 & 1081.7782 & -2.8 & HexNAc7Hex6Fuc1 \\
\hline & & 3 & 1135.7988 & 1135.7969 & -1.7 & HexNAc7Hex7Fuc1 \\
\hline & & 3 & 921.7160 & 921.7171 & 1.2 & HexNAc4Hex5Fuc1NeuAc1 \\
\hline & & 3 & 935.3915 & 935.3910 & -0.5 & HexNAc5Hex4Fuc1NeuAc1 \\
\hline & & 3 & 989.4091 & 989.4095 & 0.4 & HexNAc5Hex5Fuc1NeuAc1 \\
\hline & & 3 & 1043.4267 & 1043.4292 & 2.4 & HexNAc5Hex6Fuc1NeuAc1 \\
\hline & & 3 & 949.0670 & 949.0652 & -1.9 & HexNAc6Hex3Fuc1NeuAc1 \\
\hline & & 3 & 1192.4885 & 1192.4829 & -4.7 & HexNAc8Hex5Fuc1NeuAc1 \\
\hline & & 3 & 984.0775 & 984.0742 & -3.4 & HexNAc5Hex4Fuc2NeuAc1 \\
\hline & & 3 & 1024.0794 & 1024.0732 & -6.1 & HexNAc4Hex6NeuAc2 \\
\hline & & 2 & 1527.6180 & 1527.6218 & 2.5 & HexNAc4Hex5Fuc1NeuAc2 \\
\hline & & 3 & 1032.4233 & 1032.4182 & -4.9 & HexNAc5Hex4Fuc1NeuAc2 \\
\hline & & 3 & 1086.4409 & 1086.4401 & -0.7 & HexNAc5Hex5Fuc1NeuAc2 \\
\hline & & 3 & 1140.4585 & 1140.4567 & -1.6 & HexNAc5Hex6Fuc1NeuAc2 \\
\hline & & 3 & 1046.0988 & 1046.0974 & -1.3 & HexNAc6Hex3Fuc1NeuAc2 \\
\hline & & 3 & 1154.1340 & 1154.1318 & -1.9 & HexNAc6Hex5Fuc1NeuAc2 \\
\hline & & 3 & 1208.1516 & 1208.1526 & 0.8 & HexNAc6Hex6Fuc1NeuAc2 \\
\hline & & 3 & 1262.1692 & 1262.1661 & -2.5 & HexNAc6Hex7Fuc1NeuAc2 \\
\hline & & 3 & 1289.5203 & 1289.5168 & -2.7 & HexNAc8Hex5Fuc1NeuAc2 \\
\hline & & 3 & 1189.1445 & 1189.1449 & 0.3 & HexNAc5Hex6Fuc2NeuAc2 \\
\hline & & 3 & 1202.8200 & 1202.8202 & 0.2 & HexNAc6Hex5Fuc2NeuAc2 \\
\hline & \multirow{5}{*}{ LTPLCVTLNCTDLR } & 3 & 802.7098 & 802.7070 & -3.5 & HexNAc2Hex2 \\
\hline & & 3 & 856.7274 & 856.7257 & -2.0 & HexNAc2Hex3 \\
\hline & & 3 & 910.7450 & 910.7435 & -1.6 & HexNAc2Hex4 \\
\hline & & 3 & 964.7626 & 964.7608 & -1.9 & HexNAc2Hex5 \\
\hline & & 3 & 1018.7802 & 1018.7798 & -0.4 & HexNAc2Hex6 \\
\hline
\end{tabular}




\begin{tabular}{|c|c|c|c|c|c|c|}
\hline Domain & Peptide sequence & $\begin{array}{c}\text { Charge } \\
\text { state }\end{array}$ & $\begin{array}{c}\text { Theoretical } \\
m / z\end{array}$ & $\begin{array}{c}\text { Observed } \\
m / z\end{array}$ & $\begin{array}{c}\text { Mass } \\
\text { accurac } \\
\text { y (ppm) }\end{array}$ & Glycoform \\
\hline & & 3 & 1072.7978 & 1072.7968 & -0.9 & HexNAc2Hex7 \\
\hline & & 3 & 1126.8154 & 1126.8141 & -1.2 & HexNAc2Hex8 \\
\hline & & 2 & 1180.8330 & 1180.8309 & -1.8 & HexNAc2Hex9 \\
\hline & & 3 & 924.4206 & 924.4191 & -1.6 & HexNAc3Hex3 \\
\hline & & 3 & 978.4382 & 978.4380 & -0.2 & HexNAc3Hex4 \\
\hline & & 2 & 1548.1800 & 1548.1755 & -2.9 & HexNAc3Hex 5 \\
\hline & & 3 & 1086.4734 & 1086.4723 & -1.0 & HexNAc3Hex6 \\
\hline & & 3 & 992.1137 & 992.1155 & 1.8 & HexNAc4Hex3 \\
\hline & & 3 & 1046.1313 & 1046.1261 & -5.0 & HexNAc4Hex4 \\
\hline & & 3 & 1100.1489 & 1100.1450 & -3.5 & HexNAc4Hex5 \\
\hline & & 3 & 1221.8596 & 1221.8585 & -0.9 & HexNAc5Hex6 \\
\hline & & 3 & 905.4134 & 905.4164 & 3.3 & HexNAc2Hex3Fuc1 \\
\hline & & 2 & 1459.1562 & 1459.1564 & 0.1 & HexNAc3Hex3Fuc1 \\
\hline & & 2 & 1540.1826 & 1540.1772 & -3.5 & HexNAc3Hex4Fuc1 \\
\hline & & 3 & 1040.7997 & 1040.7897 & -9.6 & HexNAc4Hex3Fuc1 \\
\hline & & 3 & 1094.8173 & 1094.8082 & -8.3 & HexNAc4Hex4Fuc1 \\
\hline & & 3 & 1148.8349 & 1148.8328 & -1.8 & HexNAc4Hex5Fuc1 \\
\hline & & 3 & 1202.8525 & 1202.8495 & -2.5 & HexNAc4Hex6Fuc1 \\
\hline & & 3 & 1108.4928 & 1108.4899 & -2.6 & HexNAc5Hex3Fuc1 \\
\hline & & 3 & 1162.5104 & 1162.5074 & -2.6 & HexNAc5Hex4Fuc1 \\
\hline & & 3 & 1230.2035 & 1230.1992 & -3.5 & HexNAc6Hex4Fuc1 \\
\hline & & 3 & 1284.2211 & 1284.2209 & -0.2 & HexNAc6Hex5Fuc1 \\
\hline & & 3 & 1297.8967 & 1297.8877 & -6.9 & HexNAc7Hex4Fuc1 \\
\hline & & 3 & 1351.9143 & 1351.9120 & -1.7 & HexNAc7Hex5Fuc1 \\
\hline & & 4 & 1095.2140 & 1095.2123 & -1.6 & HexNAc7Hex7Fuc1 \\
\hline & & 3 & 1197.5208 & 1197.5151 & -4.8 & HexNAc4Hex5Fuc2 \\
\hline & & 3 & 1075.4700 & 1075.4696 & -0.4 & HexNAc3Hex4NeuAc1 \\
\hline & & 3 & 1129.4876 & 1129.4858 & -1.6 & HexNAc3Hex5NeuAc1 \\
\hline & & 3 & 1183.5052 & 1183.5053 & 0.1 & HexNAc3Hex6NeuAc1 \\
\hline & & 3 & 1197.1807 & 1197.1786 & -1.8 & HexNAc4Hex5NeuAc1 \\
\hline & & 3 & 1251.1983 & 1251.1939 & -3.5 & HexNAc4Hex6NeuAc1 \\
\hline & & 3 & 1305.2159 & 1305.2111 & -3.7 & HexNAc4Hex7NeuAc1 \\
\hline & & 3 & 1318.8914 & 1318.8932 & 1.4 & HexNAc5Hex6NeuAc1 \\
\hline & & 3 & 1332.5670 & 1332.5690 & 1.5 & HexNAc6Hex5NeuAc1 \\
\hline & & 3 & 1386.5846 & 1386.5806 & -2.9 & HexNAc6Hex6NeuAc1 \\
\hline & & 3 & 1124.1559 & 1124.1542 & -1.5 & HexNAc3Hex4Fuc1NeuAc1 \\
\hline & & 3 & 1178.1735 & 1178.1719 & -1.4 & HexNAc3Hex5Fuc1NeuAc1 \\
\hline & & 3 & 1232.1911 & 1232.1884 & -2.2 & HexNAc3Hex6Fuc1NeuAc1 \\
\hline & & 3 & 1245.8667 & 1245.8651 & -1.3 & HexNAc4Hex5Fuc1NeuAc1 \\
\hline & & 3 & 1299.8843 & 1299.8797 & -3.5 & HexNAc4Hex6Fuc1NeuAc1 \\
\hline & & 3 & 985.4217 & 985.4160 & -5.8 & HexNAc5Hex5Fuc1NeuAc1 \\
\hline & & 3 & 1367.5774 & 1367.5742 & -2.3 & HexNAc5Hex6Fuc1NeuAc1 \\
\hline & & 4 & 1117.2179 & 1117.2157 & -2.0 & HexNAc6Hex7Fuc1NeuAc1 \\
\hline
\end{tabular}




\begin{tabular}{|c|c|c|c|c|c|c|}
\hline Domain & Peptide sequence & $\begin{array}{c}\text { Charge } \\
\text { state }\end{array}$ & $\begin{array}{c}\text { Theoretical } \\
\mathrm{m} / z\end{array}$ & $\begin{array}{c}\text { Observed } \\
m / z\end{array}$ & $\begin{array}{c}\text { Mass } \\
\text { accurac } \\
\text { y (ppm) }\end{array}$ & Glycoform \\
\hline & & 3 & 1294.2125 & 1294.2149 & 1.9 & HexNAc4Hex5NeuAc2 \\
\hline & & 3 & 1348.2301 & 1348.2249 & -3.9 & HexNAc4Hex6NeuAc2 \\
\hline \multirow{28}{*}{$\mathrm{V} 1 / \mathrm{V} 2$} & \multirow{28}{*}{$\underline{\text { NCSFNITTNIR }}$} & 3 & 798.6756 & 798.6749 & -0.9 & HexNAc2Hex4 \\
\hline & & 3 & 852.6932 & 852.6916 & -1.9 & HexNAc2Hex5 \\
\hline & & 3 & 906.7108 & 906.7089 & -2.1 & HexNAc2Hex6 \\
\hline & & 3 & 960.7284 & 960.7267 & -1.8 & HexNAc2Hex7 \\
\hline & & 3 & 1014.7460 & 1014.7451 & -0.9 & HexNAc2Hex8 \\
\hline & & 3 & 920.3863 & 920.3832 & -3.4 & HexNAc3Hex5 \\
\hline & & 3 & 974.4039 & 974.4020 & -1.9 & HexNAc3Hex6 \\
\hline & & 3 & 988.0795 & 988.0754 & -4.1 & HexNAc4Hex5 \\
\hline & & 3 & 1258.1675 & 1258.1644 & -2.5 & HexNAc4Hex 10 \\
\hline & & 3 & 1312.1851 & 1312.1806 & -3.4 & HexNAc4Hex11 \\
\hline & & 3 & 1366.2027 & 1366.1974 & -3.9 & HexNAc4Hex12 \\
\hline & & 3 & 1420.2203 & 1420.2192 & -0.8 & HexNAc4Hex13 \\
\hline & & 3 & 1474.2379 & 1474.2371 & -0.5 & HexNAc4Hex14 \\
\hline & & 3 & 1528.2555 & 1528.2500 & -3.6 & HexNAc4Hex 15 \\
\hline & & 3 & 1582.2731 & 1582.2697 & -2.1 & HexNAc4Hex16 \\
\hline & & 3 & 969.0723 & 969.0746 & 2.4 & HexNAc3Hex5Fuc1 \\
\hline & & 3 & 1023.0899 & 1023.0840 & -5.8 & HexNAc3Hex6Fuc1 \\
\hline & & 3 & 1036.7654 & 1036.7606 & -4.6 & HexNAc4Hex5Fuc1 \\
\hline & & 3 & 1064.1165 & 1064.1173 & 0.8 & HexNAc6Hex3Fuc1 \\
\hline & & 3 & 1172.1517 & 1172.1548 & 2.6 & HexNAc6Hex5Fuc1 \\
\hline & & 3 & 1226.1693 & 1226.1648 & -3.7 & HexNAc6Hex6Fuc1 \\
\hline & & 3 & 1280.1869 & 1280.1834 & -2.7 & HexNAc6Hex7Fuc1 \\
\hline & & 3 & 1347.8800 & 1347.8809 & 0.7 & HexNAc7Hex7Fuc1 \\
\hline & & 4 & 1223.9845 & 1223.9800 & -3.7 & HexNAc8Hex11Fuc1 \\
\hline & & 3 & 1017.4181 & 1017.4160 & -2.1 & HexNAc3Hex5NeuAc1 \\
\hline & & 3 & 1071.4357 & 1071.4364 & 0.7 & HexNAc3Hex6NeuAc1 \\
\hline & & 3 & 1133.7972 & 1133.7974 & 0.2 & HexNAc4Hex5Fuc1NeuAc1 \\
\hline & & 3 & 1215.1659 & 1215.1731 & 5.9 & HexNAc6Hex4Fuc1NeuAc1 \\
\hline \multirow{13}{*}{$\mathrm{C} 2$} & \multirow{13}{*}{ LISCNTSVITQACPK } & 3 & 916.0767 & 916.0757 & -1.1 & HexNAc2Hex4 \\
\hline & & 3 & 970.0943 & 970.0881 & -6.4 & HexNAc2Hex 5 \\
\hline & & 2 & 1535.6642 & 1535.6636 & -0.4 & HexNAc2Hex6 \\
\hline & & 2 & 1616.6906 & 1616.6777 & -8.0 & HexNAc2Hex7 \\
\hline & & 2 & 1697.7170 & 1697.7175 & 0.3 & HexNAc2Hex 8 \\
\hline & & 3 & 929.7522 & 929.7512 & -1.1 & HexNAc3Hex3 \\
\hline & & 3 & 983.7698 & 983.7651 & -4.8 & HexNAc3Hex 4 \\
\hline & & 3 & 1037.7874 & 1037.7844 & -2.9 & HexNAc3Hex 5 \\
\hline & & 3 & 1091.8050 & 1091.8030 & -1.8 & HexNAc3Hex6 \\
\hline & & 3 & 997.4453 & 997.4472 & 1.9 & HexNAc4Hex3 \\
\hline & & 3 & 1051.4629 & 1051.4592 & -3.5 & HexNAc4Hex4 \\
\hline & & 3 & 1105.4805 & 1105.4771 & -3.1 & HexNAc4Hex5 \\
\hline & & 3 & 1119.1561 & 1119.1509 & -4.6 & HexNAc5Hex 4 \\
\hline
\end{tabular}




\begin{tabular}{|c|c|c|c|c|c|c|}
\hline Domain & Peptide sequence & $\begin{array}{c}\text { Charge } \\
\text { state }\end{array}$ & $\begin{array}{c}\text { Theoretical } \\
m / z\end{array}$ & $\begin{array}{c}\text { Observed } \\
m / z\end{array}$ & $\begin{array}{c}\text { Mass } \\
\text { accurac } \\
\text { y (ppm) }\end{array}$ & Glycoform \\
\hline & & 3 & 1173.1737 & 1173.1716 & -1.8 & HexNAc5Hex5 \\
\hline & & 3 & 1227.1913 & 1227.1912 & -0.1 & HexNAc5Hex6 \\
\hline & & 4 & 1011.9283 & 1011.9245 & -3.8 & HexNAc6Hex7 \\
\hline & & 3 & 910.7450 & 910.7433 & -1.9 & HexNAc2Hex3Fuc1 \\
\hline & & 3 & 964.7626 & 964.7622 & -0.4 & HexNAc2Hex4Fuc1 \\
\hline & & 3 & 978.4382 & 978.4370 & -1.2 & HexNAc3Hex3Fuc1 \\
\hline & & 3 & 1032.4558 & 1032.4539 & -1.8 & HexNAc3Hex4Fuc1 \\
\hline & & 3 & 1086.4734 & 1086.4717 & -1.6 & HexNAc3Hex5Fuc1 \\
\hline & & 3 & 1140.4910 & 1140.4897 & -1.1 & HexNAc3Hex6Fuc1 \\
\hline & & 3 & 1046.1313 & 1046.1280 & -3.2 & HexNAc4Hex3Fuc1 \\
\hline & & 3 & 1100.1489 & 1100.1468 & -1.9 & HexNAc4Hex4Fuc1 \\
\hline & & 3 & 1154.1665 & 1154.1651 & -1.2 & HexNAc4Hex5Fuc1 \\
\hline & & 3 & 1208.1841 & 1208.1832 & -0.7 & HexNAc4Hex6Fuc1 \\
\hline & & 3 & 1167.8420 & 1167.8335 & -7.3 & HexNAc5Hex4Fuc1 \\
\hline & & 3 & 1221.8596 & 1221.8590 & -0.5 & HexNAc5Hex5Fuc1 \\
\hline & & 3 & 1275.8772 & 1275.8730 & -3.3 & HexNAc5Hex6Fuc1 \\
\hline & & 3 & 1181.5176 & 1181.5162 & -1.2 & HexNAc6Hex3Fuc1 \\
\hline & & 3 & 1235.5352 & 1235.5319 & -2.7 & HexNAc6Hex4Fuc1 \\
\hline & & 3 & 1289.5528 & 1289.5501 & -2.1 & HexNAc6Hex5Fuc1 \\
\hline & & 3 & 1343.5704 & 1343.5653 & -3.8 & HexNAc6Hex6Fuc1 \\
\hline & & 4 & 1048.4428 & 1048.4414 & -1.3 & HexNAc6Hex7Fuc1 \\
\hline & & 4 & 1058.6994 & 1058.6956 & -3.6 & HexNAc7Hex6Fuc1 \\
\hline & & 4 & 1099.2126 & 1099.2089 & -3.4 & HexNAc7Hex7Fuc1 \\
\hline & & 3 & 1081.1417 & 1081.1477 & 5.5 & HexNAc3Hex4Fuc2 \\
\hline & & 3 & 1135.1593 & 1135.1547 & -4.1 & HexNAc3Hex5Fuc2 \\
\hline & & 3 & 1148.8349 & 1148.8320 & -2.5 & HexNAc4Hex4Fuc2 \\
\hline & & 3 & 1202.8525 & 1202.8508 & -1.4 & HexNAc4Hex5Fuc2 \\
\hline & & 3 & 1216.5280 & 1216.5269 & -0.9 & HexNAc5Hex4Fuc2 \\
\hline & & 3 & 1270.5456 & 1270.5424 & -2.5 & HexNAc5Hex5Fuc2 \\
\hline & & 3 & 1324.5632 & 1324.5585 & -3.5 & HexNAc5Hex6Fuc2 \\
\hline & & 4 & 922.9045 & 922.9038 & -0.8 & HexNAc6Hex3Fuc2 \\
\hline & & 3 & 1338.2387 & 1338.2329 & -4.3 & HexNAc6Hex5Fuc2 \\
\hline & & 4 & 1084.9573 & 1084.9566 & -0.6 & HexNAc6Hex7Fuc2 \\
\hline & & 4 & 1095.2139 & 1095.2196 & 5.2 & HexNAc7Hex6Fuc2 \\
\hline & & 3 & 1265.2140 & 1265.2124 & -1.3 & HexNAc5Hex4Fuc3 \\
\hline & & 3 & 1278.8895 & 1278.8888 & -0.5 & HexNAc6Hex3Fuc3 \\
\hline & & 3 & 1080.8016 & 1080.8005 & -1.0 & HexNAc3Hex4NeuAc1 \\
\hline & & 3 & 1134.8192 & 1134.8167 & -2.2 & HexNAc3Hex5NeuAc1 \\
\hline & & 3 & 1188.8368 & 1188.8348 & -1.7 & HexNAc3Hex6NeuAc1 \\
\hline & & 3 & 1202.5123 & 1202.5096 & -2.2 & HexNAc4Hex5NeuAc1 \\
\hline & & 3 & 1324.2231 & 1324.2252 & 1.6 & HexNAc5Hex6NeuAc1 \\
\hline & & 3 & 1129.4876 & 1129.4840 & -3.2 & HexNAc3Hex4Fuc1NeuAc1 \\
\hline & & 3 & 1183.5052 & 1183.5017 & -3.0 & HexNAc3Hex5Fuc1NeuAc1 \\
\hline
\end{tabular}




\begin{tabular}{|c|c|c|c|c|c|c|}
\hline Domain & Peptide sequence & $\begin{array}{c}\text { Charge } \\
\text { state }\end{array}$ & $\begin{array}{c}\text { Theoretical } \\
\mathrm{m} / z\end{array}$ & $\begin{array}{c}\text { Observed } \\
m / z\end{array}$ & $\begin{array}{c}\text { Mass } \\
\text { accurac } \\
\text { y (ppm) }\end{array}$ & Glycoform \\
\hline & & 3 & 1237.5228 & 1237.5209 & -1.5 & HexNAc3Hex6Fuc1NeuAc1 \\
\hline & & 3 & 1197.1807 & 1197.1768 & -3.3 & HexNAc4Hex4Fuc1NeuAc1 \\
\hline & & 3 & 1251.1983 & 1251.1957 & -2.1 & HexNAc4Hex5Fuc1NeuAc1 \\
\hline & & 3 & 1305.2159 & 1305.2194 & 2.7 & HexNAc4Hex6Fuc1NeuAc1 \\
\hline & & 3 & 1264.8738 & 1264.8695 & -3.4 & HexNAc5Hex4Fuc1NeuAc1 \\
\hline & & 3 & 1318.8914 & 1318.8875 & -3.0 & HexNAc5Hex5Fuc1NeuAc1 \\
\hline & & 3 & 1372.9090 & 1372.9049 & -3.0 & HexNAc5Hex6Fuc1NeuAc1 \\
\hline & & 3 & 1278.5494 & 1278.5475 & -1.5 & HexNAc6Hex3Fuc1NeuAc1 \\
\hline & & 4 & 1332.5670 & 1332.5604 & -5.0 & HexNAc6Hex4Fuc1NeuAc1 \\
\hline & & 3 & 1386.5846 & 1386.5796 & -3.6 & HexNAc6Hex5Fuc1NeuAc1 \\
\hline & & 3 & 1440.6022 & 1440.5975 & -3.3 & HexNAc6Hex6Fuc1NeuAc1 \\
\hline & & 4 & 1121.2166 & 1121.2123 & -3.8 & HexNAc6Hex7Fuc1NeuAc1 \\
\hline & & 4 & 1050.4469 & 1050.4447 & -2.1 & HexNAc7Hex4Fuc1NeuAc1 \\
\hline & & 4 & 1171.9865 & 1171.9825 & -3.4 & HexNAc7Hex7Fuc1NeuAc1 \\
\hline & & 3 & 1299.8843 & 1299.8811 & -2.5 & HexNAc4Hex5Fuc2NeuAc1 \\
\hline & & 3 & 1313.5598 & 1313.5586 & -0.9 & HexNAc5Hex4Fuc2NeuAc1 \\
\hline & & 3 & 1367.5774 & 1367.5758 & -1.2 & HexNAc5Hex5Fuc2NeuAc1 \\
\hline & & 3 & 1421.5950 & 1421.5975 & 1.8 & HexNAc5Hex6Fuc2NeuAc1 \\
\hline & & 4 & 1157.7311 & 1157.7268 & -3.7 & HexNAc6Hex7Fuc2NeuAc1 \\
\hline & & 3 & 1299.5441 & 1299.5476 & 2.7 & HexNAc4Hex5NeuAc2 \\
\hline & & 3 & 1348.2301 & 1348.2250 & -3.8 & HexNAc4Hex5Fuc1NeuAc2 \\
\hline & & 3 & 1361.9056 & 1361.9012 & -3.2 & HexNAc5Hex4Fuc1NeuAc2 \\
\hline & & 3 & 1469.9408 & 1469.9398 & -0.7 & HexNAc5Hex6Fuc1NeuAc2 \\
\hline & & 3 & 1483.6164 & 1483.6173 & 0.6 & HexNAc6Hex5Fuc1NeuAc2 \\
\hline & & 3 & 1537.6340 & 1537.6318 & -1.4 & HexNAc6Hex6Fuc1NeuAc2 \\
\hline & & 4 & 1193.9905 & 1193.9867 & -3.2 & HexNAc6Hex7Fuc1NeuAc2 \\
\hline & & 4 & 1230.5050 & 1230.5035 & -1.2 & HexNAc6Hex7Fuc2NeuAc2 \\
\hline & & 4 & 1244.7603 & 1244.7555 & -3.9 & HexNAc7Hex7Fuc1NeuAc2 \\
\hline & \multirow{15}{*}{ GPCTNVSTVQCTHGIR } & 3 & 893.7213 & 893.7212 & -0.1 & HexNAc2Hex3 \\
\hline & & 3 & 947.7389 & 947.7381 & -0.8 & HexNAc2Hex 4 \\
\hline & & 3 & 1001.7565 & 1001.7555 & -1.0 & HexNAc2Hex 5 \\
\hline & & 3 & 1055.7741 & 1055.7728 & -1.2 & HexNAc2Hex6 \\
\hline & & 3 & 1109.7917 & 1109.7900 & -1.5 & HexNAc2Hex7 \\
\hline & & 3 & 1163.8093 & 1163.8070 & -2.0 & HexNAc2Hex8 \\
\hline & & 3 & 1217.8269 & 1217.8246 & -1.9 & HexNAc2Hex9 \\
\hline & & 3 & 961.4145 & 961.4151 & 0.6 & HexNAc3Hex3 \\
\hline & & 3 & 1069.4497 & 1069.4471 & -2.4 & HexNAc3Hex 5 \\
\hline & & 3 & 1123.4673 & 1123.4659 & -1.2 & HexNAc3Hex6 \\
\hline & & 3 & 1083.1252 & 1083.1224 & -2.6 & HexNAc4Hex4 \\
\hline & & 3 & 1137.1428 & 1137.1410 & -1.6 & HexNAc4Hex 5 \\
\hline & & 3 & 1191.1604 & 1191.1563 & -3.4 & HexNAc4Hex6 \\
\hline & & 3 & 1096.8007 & 1096.8004 & -0.3 & HexNAc5Hex3 \\
\hline & & 3 & 1150.8183 & 1150.8176 & -0.6 & HexNAc5Hex4 \\
\hline
\end{tabular}




\begin{tabular}{|c|c|c|c|c|c|c|}
\hline Domain & Peptide sequence & $\begin{array}{c}\begin{array}{c}\text { Charge } \\
\text { state }\end{array} \\
\end{array}$ & $\begin{array}{c}\text { Theoretical } \\
m / z\end{array}$ & $\begin{array}{c}\text { Observed } \\
m / z\end{array}$ & $\begin{array}{c}\text { Mass } \\
\text { accurac } \\
\text { y (ppm) }\end{array}$ & Glycoform \\
\hline & & 3 & 1204.8359 & 1204.8323 & -3.0 & HexNAc5Hex5 \\
\hline & & 3 & 1258.8535 & 1258.8518 & -1.4 & HexNAc5Hex6 \\
\hline & & 3 & 1272.5291 & 1272.5267 & -1.9 & HexNAc6Hex5 \\
\hline & & 3 & 1326.5467 & 1326.5422 & -3.4 & HexNAc6Hex6 \\
\hline & & 3 & 1380.5643 & 1380.5588 & -4.0 & HexNAc6Hex7 \\
\hline & & 3 & 1064.1180 & 1064.1158 & -2.1 & HexNAc3Hex4Fuc1 \\
\hline & & 3 & 1118.1356 & 1118.1349 & -0.6 & HexNAc3Hex5Fuc1 \\
\hline & & 3 & 1172.1532 & 1172.1495 & -3.2 & HexNAc3Hex6Fuc1 \\
\hline & & 3 & 1131.8112 & 1131.8109 & -0.3 & HexNAc4Hex4Fuc1 \\
\hline & & 3 & 1185.8288 & 1185.8264 & -2.0 & HexNAc4Hex5Fuc1 \\
\hline & & 3 & 1239.8464 & 1239.8416 & -3.9 & HexNAc4Hex6Fuc1 \\
\hline & & 3 & 1293.8640 & 1293.8583 & -4.4 & HexNAc4Hex7Fuc1 \\
\hline & & 3 & 1145.4867 & 1145.4866 & -0.1 & HexNAc5Hex3Fuc1 \\
\hline & & 3 & 1199.5043 & 1199.5013 & -2.5 & HexNAc5Hex4Fuc1 \\
\hline & & 3 & 1253.5219 & 1253.5223 & 0.3 & HexNAc5Hex5Fuc1 \\
\hline & & 3 & 1307.5395 & 1307.5360 & -2.7 & HexNAc5Hex6Fuc1 \\
\hline & & 3 & 1267.1974 & 1267.2030 & 4.4 & HexNAc6Hex4Fuc1 \\
\hline & & 3 & 1321.2150 & 1321.2158 & 0.6 & HexNAc6Hex5Fuc1 \\
\hline & & 3 & 1375.2326 & 1375.2354 & 2.0 & HexNAc6Hex6Fuc1 \\
\hline & & 3 & 1429.2502 & 1429.2480 & -1.5 & HexNAc6Hex7Fuc1 \\
\hline & & 3 & 1248.1903 & 1248.1919 & 1.3 & HexNAc5Hex4Fuc2 \\
\hline & & 3 & 1112.4639 & 1112.4622 & -1.5 & HexNAc3Hex4NeuAc1 \\
\hline & & 3 & 1166.4815 & 1166.4797 & -1.5 & HexNAc3Hex5NeuAc1 \\
\hline & & 3 & 1220.4991 & 1220.4967 & -2.0 & HexNAc3Hex6NeuAc1 \\
\hline & & 3 & 1180.1570 & 1180.1504 & -5.6 & HexNAc4Hex4NeuAc1 \\
\hline & & 3 & 1234.1746 & 1234.1713 & -2.7 & HexNAc4Hex5NeuAc1 \\
\hline & & 3 & 1355.8853 & 1355.8879 & 1.9 & HexNAc5Hex6NeuAc1 \\
\hline & & 3 & 1161.1498 & 1161.1487 & -0.9 & HexNAc3Hex4Fuc1NeuAc1 \\
\hline & & 3 & 1215.1674 & 1215.1641 & -2.7 & HexNAc3Hex5Fuc1NeuAc1 \\
\hline & & 3 & 1269.1850 & 1269.1802 & -3.8 & HexNAc3Hex6Fuc1NeuAc1 \\
\hline & & 3 & 1282.8606 & 1282.8582 & -1.9 & HexNAc4Hex5Fuc1NeuAc1 \\
\hline & & 3 & 1390.8958 & 1390.8922 & -2.6 & HexNAc4Hex7Fuc1NeuAc1 \\
\hline & & 3 & 1296.5361 & 1296.5365 & 0.3 & HexNAc5Hex4Fuc1NeuAc1 \\
\hline & & 3 & 1350.5537 & 1350.5504 & -2.4 & HexNAc5Hex5Fuc1NeuAc1 \\
\hline & & 3 & 1404.5713 & 1404.5769 & 4.0 & HexNAc5Hex6Fuc1NeuAc1 \\
\hline & & 3 & 1418.2468 & 1418.2435 & -2.3 & HexNAc6Hex5Fuc1NeuAc1 \\
\hline & & 3 & 1472.2644 & 1472.2600 & -3.0 & HexNAc6Hex6Fuc1NeuAc1 \\
\hline & & 3 & 1526.2820 & 1526.2870 & 3.3 & HexNAc6Hex7Fuc1NeuAc1 \\
\hline & \multirow{5}{*}{$\begin{array}{l}\text { PVVSTQLLLNGSLAEE } \\
\text { E }\end{array}$} & 3 & 1005.7935 & 1005.7900 & -3.5 & HexNAc2Hex 5 \\
\hline & & 3 & 1059.8111 & 1059.8097 & -1.3 & HexNAc2Hex6 \\
\hline & & 3 & 1113.8287 & 1113.8273 & -1.3 & HexNAc2Hex7 \\
\hline & & 3 & 1167.8463 & 1167.8431 & -2.8 & HexNAc2Hex8 \\
\hline & & 3 & 1221.8639 & 1221.8623 & -1.3 & HexNAc2Hex9 \\
\hline
\end{tabular}




\begin{tabular}{|c|c|c|c|c|c|c|}
\hline Domain & Peptide sequence & $\begin{array}{c}\text { Charge } \\
\text { state }\end{array}$ & $\begin{array}{c}\text { Theoretical } \\
m / z\end{array}$ & $\begin{array}{c}\text { Observed } \\
m / z\end{array}$ & $\begin{array}{c}\text { Mass } \\
\text { accurac } \\
\text { y (ppm) }\end{array}$ & Glycoform \\
\hline & \multirow{7}{*}{ VIIVQLNEE(S) } & 2 & 910.4377 & 910.4369 & -0.9 & HexNAc2Hex3 \\
\hline & & 2 & 991.4641 & 991.4625 & -1.6 & HexNAc2Hex4 \\
\hline & & 2 & 1072.4905 & 1072.4893 & -1.1 & HexNAc2Hex 5 \\
\hline & & 2 & 1153.5169 & 1153.5159 & -0.9 & HexNAc2Hex6 \\
\hline & & 2 & 1234.5433 & 1234.5418 & -1.2 & HexNAc2Hex7 \\
\hline & & 2 & 1315.5697 & 1315.5673 & -1.8 & HexNAc2Hex 8 \\
\hline & & 2 & 1396.5961 & 1396.5938 & -1.6 & HexNAc2Hex9 \\
\hline \multirow{36}{*}{$\mathrm{C} 3$} & \multirow{31}{*}{ QAHCNLSR } & 3 & 896.6863 & 896.6886 & 2.6 & HexNAc2Hex8 \\
\hline & & 3 & 950.7039 & 950.7064 & 2.6 & HexNAc2Hex9 \\
\hline & & 3 & 1005.4060 & 1005.4087 & 2.7 & HexNAc6Hex5 \\
\hline & & 3 & 1059.4236 & 1059.4269 & 3.1 & HexNAc6Hex6 \\
\hline & & 3 & 1127.1168 & 1127.1208 & 3.5 & HexNAc7Hex6 \\
\hline & & 3 & 851.0126 & 851.0162 & 4.2 & HexNAc3Hex5Fuc1 \\
\hline & & 3 & 864.6881 & 864.6915 & 3.9 & HexNAc4Hex4Fuc1 \\
\hline & & 3 & 918.7057 & 918.7079 & 2.4 & HexNAc4Hex5Fuc1 \\
\hline & & 3 & 878.3637 & 878.3642 & 0.6 & HexNAc5Hex3Fuc1 \\
\hline & & 3 & 932.3813 & 932.3826 & 1.4 & HexNAc5Hex4Fuc1 \\
\hline & & 3 & 986.3989 & 986.4012 & 2.3 & HexNAc5Hex5Fuc1 \\
\hline & & 3 & 1040.4165 & 1040.4186 & 2.0 & HexNAc5Hex6Fuc1 \\
\hline & & 3 & 1000.0744 & 1000.0745 & 0.1 & HexNAc6Hex4Fuc1 \\
\hline & & 3 & 1054.0920 & 1054.0942 & 2.1 & HexNAc6Hex5Fuc1 \\
\hline & & 3 & 1108.1096 & 1108.1121 & 2.3 & HexNAc6Hex6Fuc1 \\
\hline & & 3 & 1162.1272 & 1162.1292 & 1.7 & HexNAc6Hex7Fuc1 \\
\hline & & 3 & 1067.7675 & 1067.7698 & 2.2 & HexNAc7Hex4Fuc1 \\
\hline & & 3 & 1121.7851 & 1121.7852 & 0.1 & HexNAc7Hex5Fuc1 \\
\hline & & 3 & 1175.8027 & 1175.8046 & 1.6 & HexNAc7Hex6Fuc1 \\
\hline & & 3 & 1229.8203 & 1229.8220 & 1.4 & HexNAc7Hex7Fuc1 \\
\hline & & 3 & 1156.4554 & 1156.4609 & 4.8 & HexNAc6Hex6NeuAc1 \\
\hline & & 3 & 1015.7375 & 1015.7397 & 2.2 & HexNAc4Hex5Fuc1NeuAc1 \\
\hline & & 3 & 1029.4131 & 1029.4135 & 0.4 & HexNAc5Hex4Fuc1NeuAc1 \\
\hline & & 3 & 1083.4307 & 1083.4327 & 1.8 & HexNAc5Hex5Fuc1NeuAc1 \\
\hline & & 3 & 1137.4483 & 1137.4513 & 2.6 & HexNAc5Hex6Fuc1NeuAc1 \\
\hline & & 3 & 1097.1062 & 1097.1056 & -0.5 & HexNAc6Hex4Fuc1NeuAc1 \\
\hline & & 3 & 1151.1238 & 1151.1246 & 0.7 & HexNAc6Hex5Fuc1NeuAc1 \\
\hline & & 3 & 1205.1414 & 1205.1399 & -1.2 & HexNAc6Hex6Fuc1NeuAc1 \\
\hline & & 3 & 1259.1590 & 1259.1602 & 1.0 & HexNAc6Hex7Fuc1NeuAc1 \\
\hline & & 3 & 954.8777 & 954.8774 & -0.3 & HexNAc7Hex6Fuc1NeuAc1 \\
\hline & & 3 & 995.3909 & 995.3962 & 5.3 & HexNAc7Hex7Fuc1NeuAc1 \\
\hline & & 2 & 972.9069 & 972.9050 & -2.0 & HexNAc2Hex 4 \\
\hline & & 2 & 1053.9334 & 1053.9315 & -1.8 & HexNAc2Hex 5 \\
\hline & WNDTLNK & 2 & 1134.9598 & 1134.9575 & -2.0 & HexNAc2Hex6 \\
\hline & & 2 & 1215.9862 & 1215.9842 & -1.6 & HexNAc2Hex7 \\
\hline & & 2 & 1297.0126 & 1297.0106 & -1.5 & HexNAc2Hex8 \\
\hline
\end{tabular}




\begin{tabular}{|c|c|c|c|c|c|c|}
\hline Domain & Peptide sequence & $\begin{array}{c}\text { Charge } \\
\text { state }\end{array}$ & $\begin{array}{c}\text { Theoretical } \\
m / z\end{array}$ & $\begin{array}{c}\text { Observed } \\
m / z\end{array}$ & $\begin{array}{c}\text { Mass } \\
\text { accurac } \\
\text { y (ppm) }\end{array}$ & Glycoform \\
\hline & & 2 & 1378.0390 & 1378.0361 & -2.1 & HexNAc2Hex9 \\
\hline & & 2 & 993.4203 & 993.4224 & 2.1 & HexNAc3Hex3 \\
\hline & & 2 & 1074.4467 & 1074.4458 & -0.8 & HexNAc3Hex4 \\
\hline & & 2 & 1155.4731 & 1155.4709 & -1.9 & HexNAc3Hex 5 \\
\hline & & 2 & 1236.4994 & 1236.5000 & 0.5 & HexNAc3Hex6 \\
\hline & & 2 & 1094.9600 & 1094.9595 & -0.5 & HexNAc4Hex3 \\
\hline & & 2 & 1175.9864 & 1175.9849 & -1.3 & HexNAc4Hex4 \\
\hline & & 2 & 1257.0128 & 1257.0094 & -2.7 & HexNAc4Hex5 \\
\hline & & 2 & 1066.4492 & 1066.4481 & -1.0 & HexNAc3Hex3Fuc1 \\
\hline & & 2 & 1147.4756 & 1147.4734 & -1.9 & HexNAc3Hex4Fuc1 \\
\hline & & 2 & 1228.5020 & 1228.5000 & -1.6 & HexNAc3Hex5Fuc1 \\
\hline & & 2 & 1309.5284 & 1309.5254 & -2.3 & HexNAc3Hex6Fuc1 \\
\hline & & 2 & 1167.9889 & 1167.9860 & -2.5 & HexNAc4Hex3Fuc1 \\
\hline & & 2 & 1249.0153 & 1249.0144 & -0.7 & HexNAc4Hex4Fuc1 \\
\hline & & 2 & 1330.0417 & 1330.0374 & -3.2 & HexNAc4Hex5Fuc1 \\
\hline & & 2 & 1350.5550 & 1350.5504 & -3.4 & HexNAc5Hex4Fuc1 \\
\hline & & 3 & 954.7234 & 954.7231 & -0.3 & HexNAc5Hex5Fuc1 \\
\hline & & 3 & 1008.7410 & 1008.7400 & -1.0 & HexNAc5Hex6Fuc1 \\
\hline & & 3 & 1076.4341 & 1076.4337 & -0.4 & HexNAc6Hex6Fuc1 \\
\hline & & 3 & 1130.4517 & 1130.4488 & -2.6 & HexNAc6Hex7Fuc1 \\
\hline & & 3 & 1090.1096 & 1090.1176 & 7.3 & HexNAc7Hex5Fuc1 \\
\hline & & 3 & 1144.1272 & 1144.1206 & -5.8 & HexNAc7Hex6Fuc1 \\
\hline & & 3 & 1301.0208 & 1301.0171 & -2.8 & HexNAc3Hex5NeuAc1 \\
\hline & & 3 & 1382.0472 & 1382.0429 & -3.1 & HexNAc3Hex6NeuAc1 \\
\hline & & 3 & 1057.0868 & 1057.0876 & 0.8 & HexNAc5Hex6NeuAc1 \\
\hline & & 2 & 1374.0497 & 1374.0467 & -2.2 & HexNAc3Hex5Fuc1NeuAc1 \\
\hline & & 3 & 970.3865 & 970.3823 & -4.3 & HexNAc3Hex6Fuc1NeuAc1 \\
\hline & & 3 & 930.0444 & 930.0405 & -4.2 & HexNAc4Hex4Fuc1NeuAc1 \\
\hline & & 3 & 984.0620 & 984.0611 & -0.9 & HexNAc4Hex5Fuc1NeuAc1 \\
\hline & & 3 & 1038.0796 & 1038.0778 & -1.7 & HexNAc4Hex6Fuc1NeuAc1 \\
\hline & & 3 & 997.7376 & 997.7333 & -4.3 & HexNAc5Hex4Fuc1NeuAc1 \\
\hline & & 3 & 1051.7551 & 1051.7544 & -0.7 & HexNAc5Hex5Fuc1NeuAc1 \\
\hline & & 3 & 1105.7728 & 1105.7711 & -1.5 & HexNAc5Hex6Fuc1NeuAc1 \\
\hline & & 3 & 1119.4483 & 1119.4471 & -1.1 & HexNAc6Hex5Fuc1NeuAc1 \\
\hline & & 3 & 1173.4659 & 1173.4635 & -2.0 & HexNAc6Hex6Fuc1NeuAc1 \\
\hline & & 3 & 1227.4835 & 1227.4808 & -2.2 & HexNAc6Hex7Fuc1NeuAc1 \\
\hline & & 3 & 1241.1590 & 1241.1541 & -3.9 & HexNAc7Hex6Fuc1NeuAc1 \\
\hline & & 3 & 1295.1766 & 1295.1719 & -3.6 & HexNAc7Hex7Fuc1NeuAc1 \\
\hline & \multirow{5}{*}{ LREQFGNK(T) } & 2 & 1015.4572 & 1015.4597 & 2.5 & HexNAc2Hex3Fuc1 \\
\hline & & 2 & 1023.4546 & 1023.4580 & 3.3 & HexNAc2Hex4 \\
\hline & & 3 & 736.6564 & 736.6572 & 1.1 & HexNAc2Hex5 \\
\hline & & 3 & 790.6740 & 790.6754 & 1.8 & HexNAc2Hex6 \\
\hline & & 3 & 844.6916 & 844.6930 & 1.7 & HexNAc2Hex7 \\
\hline
\end{tabular}




\begin{tabular}{|c|c|c|c|c|c|c|}
\hline Domain & Peptide sequence & $\begin{array}{c}\text { Charge } \\
\text { state }\end{array}$ & $\begin{array}{c}\text { Theoretical } \\
m / z\end{array}$ & $\begin{array}{c}\text { Observed } \\
m / z\end{array}$ & $\begin{array}{c}\text { Mass } \\
\text { accurac } \\
\text { y (ppm) }\end{array}$ & Glycoform \\
\hline & & 3 & 804.3496 & 804.3500 & 0.5 & HexNAc3Hex 5 \\
\hline & & 3 & 858.3672 & 858.3679 & 0.8 & HexNAc3Hex6 \\
\hline & & 3 & 818.0251 & 818.0241 & -1.2 & HexNAc4Hex4 \\
\hline & & 3 & 872.0427 & 872.0414 & -1.5 & HexNAc4Hex 5 \\
\hline & & 3 & 926.0603 & 926.0612 & 1.0 & HexNAc4Hex6 \\
\hline & & 3 & 745.0003 & 745.0008 & 0.7 & HexNAc3Hex3Fuc1 \\
\hline & & 3 & 799.0179 & 799.0178 & -0.1 & HexNAc3Hex4Fuc1 \\
\hline & & 3 & 853.0355 & 853.0348 & -0.8 & HexNAc3Hex5Fuc1 \\
\hline & & 3 & 907.0531 & 907.0513 & -2.0 & HexNAc3Hex6Fuc1 \\
\hline & & 3 & 812.6935 & 812.6913 & -2.7 & HexNAc4Hex3Fuc1 \\
\hline & & 3 & 866.7111 & 866.7105 & -0.7 & HexNAc4Hex4Fuc1 \\
\hline & & 3 & 920.7287 & 920.7281 & -0.7 & HexNAc4Hex5Fuc1 \\
\hline & & 3 & 974.7463 & 974.7496 & 3.4 & HexNAc4Hex6Fuc1 \\
\hline & & 3 & 880.3866 & 880.3857 & -1.0 & HexNAc5Hex3Fuc1 \\
\hline & & 3 & 934.4042 & 934.4028 & -1.5 & HexNAc5Hex4Fuc1 \\
\hline & & 3 & 988.4218 & 988.4205 & -1.3 & HexNAc5Hex5Fuc1 \\
\hline & & 3 & 1042.4394 & 1042.4379 & -1.4 & HexNAc5Hex6Fuc1 \\
\hline & & 3 & 948.0797 & 948.0789 & -0.8 & HexNAc6Hex3Fuc1 \\
\hline & & 3 & 1002.0973 & 1002.0962 & -1.1 & HexNAc6Hex4Fuc1 \\
\hline & & 3 & 1056.1149 & 1056.1134 & -1.4 & HexNAc6Hex5Fuc1 \\
\hline & & 3 & 1164.1501 & 1164.1492 & -0.8 & HexNAc6Hex7Fuc1 \\
\hline & & 3 & 847.3638 & 847.3657 & 2.2 & HexNAc3Hex4NeuAc1 \\
\hline & & 3 & 901.3814 & 901.3797 & -1.9 & HexNAc3Hex5NeuAc1 \\
\hline & & 3 & 955.3990 & 955.3972 & -1.9 & HexNAc3Hex6NeuAc1 \\
\hline & & 3 & 915.0569 & 915.0562 & -0.8 & HexNAc4Hex4NeuAc1 \\
\hline & & 3 & 969.0745 & 969.0734 & -1.1 & HexNAc4Hex5NeuAc1 \\
\hline & & 3 & 896.0497 & 896.0482 & -1.7 & HexNAc3Hex4Fuc1NeuAc1 \\
\hline & & 3 & 950.0673 & 950.0645 & -2.9 & HexNAc3Hex5Fuc1NeuAc1 \\
\hline & & 3 & 1004.0849 & 1004.0831 & -1.8 & HexNAc3Hex6Fuc1NeuAc1 \\
\hline & & 3 & 963.7429 & 963.7410 & -2.0 & HexNAc4Hex4Fuc1NeuAc1 \\
\hline & & 3 & 1017.7605 & 1017.7589 & -1.6 & HexNAc4Hex5Fuc1NeuAc1 \\
\hline & & 3 & 1071.7781 & 1071.7733 & -4.5 & HexNAc4Hex6Fuc1NeuAc1 \\
\hline & & 3 & 1031.4360 & 1031.4393 & 3.2 & HexNAc5Hex4Fuc1NeuAc1 \\
\hline & & 3 & 1085.4536 & 1085.4514 & -2.0 & HexNAc5Hex5Fuc1NeuAc1 \\
\hline & & 3 & 1139.4712 & 1139.4689 & -2.0 & HexNAc5Hex6Fuc1NeuAc1 \\
\hline & & 3 & 1099.1291 & 1099.1285 & -0.5 & HexNAc6Hex4Fuc1NeuAc1 \\
\hline & & 3 & 1153.1467 & 1153.1462 & -0.4 & HexNAc6Hex5Fuc1NeuAc1 \\
\hline & & 3 & 1207.1643 & 1207.1599 & -3.6 & HexNAc6Hex6Fuc1NeuAc1 \\
\hline & & 3 & 1261.1819 & 1261.1764 & -4.4 & HexNAc6Hex7Fuc1NeuAc1 \\
\hline & & 3 & 1114.7923 & 1114.7933 & 0.9 & HexNAc4Hex5Fuc1NeuAc2 \\
\hline & & 3 & 1168.8099 & 1168.8118 & 1.6 & HexNAc4Hex6Fuc1NeuAc2 \\
\hline & & 3 & 1182.4854 & 1182.4833 & -1.8 & HexNAc5Hex5Fuc1NeuAc2 \\
\hline & & 3 & 1236.5030 & 1236.5006 & -1.9 & HexNAc5Hex6Fuc1NeuAc2 \\
\hline
\end{tabular}




\begin{tabular}{|c|c|c|c|c|c|c|}
\hline Domain & Peptide sequence & $\begin{array}{c}\text { Charge } \\
\text { state }\end{array}$ & $\begin{array}{c}\text { Theoretical } \\
m / z\end{array}$ & $\begin{array}{c}\text { Observed } \\
m / z\end{array}$ & $\begin{array}{c}\text { Mass } \\
\text { accurac } \\
\text { y (ppm) }\end{array}$ & Glycoform \\
\hline & & 3 & 1304.1961 & 1304.1946 & -1.2 & HexNAc6Hex6Fuc1NeuAc2 \\
\hline & & 3 & 1358.2137 & 1358.2103 & -2.5 & HexNAc6Hex7Fuc1NeuAc2 \\
\hline \multirow{41}{*}{ V4 } & \multirow{39}{*}{ SNNTVENNTITLPCR } & 3 & 929.7376 & 929.7362 & -1.5 & HexNAc2Hex4 \\
\hline & & 3 & 983.7552 & 983.7527 & -2.5 & HexNAc2Hex 5 \\
\hline & & 3 & 1037.7728 & 1037.7709 & -1.8 & HexNAc2Hex6 \\
\hline & & 3 & 1091.7904 & 1091.7883 & -1.9 & HexNAc2Hex7 \\
\hline & & 3 & 1145.8080 & 1145.8053 & -2.4 & HexNAc2Hex8 \\
\hline & & 3 & 1199.8256 & 1199.8259 & 0.3 & HexNAc2Hex9 \\
\hline & & 3 & 997.4307 & 997.4274 & -3.3 & HexNAc3Hex 4 \\
\hline & & 3 & 1051.4483 & 1051.4464 & -1.8 & HexNAc3Hex 5 \\
\hline & & 2 & 1657.6953 & 1657.6866 & -5.2 & HexNAc3Hex6 \\
\hline & & 3 & 1119.1415 & 1119.1394 & -1.9 & HexNAc4Hex 5 \\
\hline & & 3 & 1497.2647 & 1497.2632 & -1.0 & HexNAc4Hex 12 \\
\hline & & 3 & 1551.2823 & 1551.2827 & 0.3 & HexNAc4Hex13 \\
\hline & & 4 & 1173.9702 & 1173.9650 & -4.4 & HexNAc5Hex 12 \\
\hline & & 4 & 1214.4834 & 1214.4777 & -4.7 & HexNAc5Hex13 \\
\hline & & 3 & 1046.1167 & 1046.1154 & -1.2 & HexNAc3Hex4Fuc1 \\
\hline & & 2 & 1649.6978 & 1649.6893 & -5.2 & HexNAc3Hex5Fuc1 \\
\hline & & 3 & 1154.1519 & 1154.1614 & 8.2 & HexNAc3Hex6Fuc1 \\
\hline & & 3 & 1167.8274 & 1167.8306 & 2.7 & HexNAc4Hex5Fuc1 \\
\hline & & 3 & 1127.4854 & 1127.4761 & -8.2 & HexNAc5Hex3Fuc1 \\
\hline & & 3 & 1235.5206 & 1235.5137 & -5.6 & HexNAc5Hex5Fuc1 \\
\hline & & 3 & 1289.5382 & 1289.5370 & -0.9 & HexNAc5Hex6Fuc1 \\
\hline & & 4 & 1210.4846 & 1210.4860 & 1.2 & HexNAc5Hex12Fuc1 \\
\hline & & 4 & 1250.9978 & 1250.9938 & -3.2 & HexNAc5Hex13Fuc1 \\
\hline & & 4 & 1291.5110 & 1291.5071 & -3.0 & HexNAc5Hex14Fuc1 \\
\hline & & 3 & 1303.2137 & 1303.2101 & -2.8 & HexNAc6Hex5Fuc1 \\
\hline & & 4 & 1018.1753 & 1018.1741 & -1.2 & HexNAc6Hex6Fuc1 \\
\hline & & 4 & 1180.2281 & 1180.2336 & 4.7 & HexNAc6Hex10Fuc1 \\
\hline & & 4 & 1261.2545 & 1261.2504 & -3.3 & HexNAc6Hex12Fuc1 \\
\hline & & 4 & 1301.7677 & 1301.7629 & -3.7 & HexNAc6Hex13Fuc1 \\
\hline & & 4 & 1312.0243 & 1312.0206 & -2.8 & HexNAc7Hex12Fuc1 \\
\hline & & 4 & 1352.5375 & 1352.5365 & -0.7 & HexNAc7Hex13Fuc1 \\
\hline & & 3 & 1148.4801 & 1148.4790 & -1.0 & HexNAc3Hex5NeuAc1 \\
\hline & & 3 & 1202.4977 & 1202.4952 & -2.1 & HexNAc3Hex6NeuAc1 \\
\hline & & 3 & 1216.1733 & 1216.1671 & -5.1 & HexNAc4Hex5NeuAc1 \\
\hline & & 3 & 1197.1661 & 1197.1670 & 0.8 & HexNAc3Hex5Fuc1NeuAc1 \\
\hline & & 3 & 1264.8592 & 1264.8572 & -1.6 & HexNAc4Hex5Fuc1NeuAc1 \\
\hline & & 3 & 1386.5700 & 1386.5800 & 7.2 & HexNAc5Hex6Fuc1NeuAc1 \\
\hline & & 4 & 1090.9491 & 1090.9501 & 0.9 & HexNAc6Hex6Fuc1NeuAc1 \\
\hline & & 4 & 1112.9531 & 1112.9526 & -0.4 & HexNAc5Hex6Fuc1NeuAc2 \\
\hline & \multirow{2}{*}{ NNTITLPCR } & 2 & 828.8853 & 828.8846 & -0.8 & HexNAc2Hex 1 \\
\hline & & 2 & 909.9117 & 909.9111 & -0.7 & HexNAc2Hex2 \\
\hline
\end{tabular}




\begin{tabular}{|c|c|c|c|c|c|c|}
\hline Domain & Peptide sequence & $\begin{array}{c}\begin{array}{c}\text { Charge } \\
\text { state }\end{array} \\
\end{array}$ & $\begin{array}{c}\text { Theoretical } \\
m / z\end{array}$ & $\begin{array}{c}\text { Observed } \\
m / z\end{array}$ & $\begin{array}{c}\text { Mass } \\
\text { accurac } \\
\text { y (ppm) }\end{array}$ & Glycoform \\
\hline & & 3 & 714.9787 & 714.9800 & 1.8 & HexNAc2Hex4 \\
\hline & & 3 & 768.9963 & 768.9958 & -0.7 & HexNAc2Hex 5 \\
\hline & & 3 & 823.0139 & 823.0138 & -0.1 & HexNAc2Hex6 \\
\hline & & 3 & 877.0315 & 877.0293 & -2.5 & HexNAc2Hex7 \\
\hline & & 2 & 1396.0701 & 1396.0659 & -3.0 & HexNAc2Hex 8 \\
\hline & & 2 & 1477.0965 & 1477.0903 & -4.2 & HexNAc2Hex9 \\
\hline & & 2 & 1173.5042 & 1173.5022 & -1.7 & HexNAc3Hex4 \\
\hline & & 2 & 1254.5306 & 1254.5259 & -3.7 & HexNAc3Hex5 \\
\hline & & 3 & 890.7071 & 890.7064 & -0.8 & HexNAc3Hex6 \\
\hline & & 3 & 904.3826 & 904.3800 & -2.9 & HexNAc4Hex5 \\
\hline & & 3 & 958.4002 & 958.4021 & 2.0 & HexNAc4Hex6 \\
\hline & & 3 & 864.0405 & 864.0401 & -0.5 & HexNAc5Hex3 \\
\hline & & 3 & 918.0581 & 918.0551 & -3.3 & HexNAc5Hex4 \\
\hline & & 2 & 1165.5067 & 1165.5062 & -0.4 & HexNAc3Hex3Fuc1 \\
\hline & & 2 & 1246.5331 & 1246.5365 & 2.7 & HexNAc3Hex4Fuc1 \\
\hline & & 2 & 1327.5595 & 1327.5610 & 1.1 & HexNAc3Hex5Fuc1 \\
\hline & & 3 & 939.3930 & 939.3924 & -0.6 & HexNAc3Hex6Fuc1 \\
\hline & & 3 & 993.4106 & 993.4062 & -4.4 & HexNAc3Hex7Fuc1 \\
\hline & & 2 & 1348.0728 & 1348.0642 & -6.4 & HexNAc4Hex4Fuc1 \\
\hline & & 3 & 953.0686 & 953.0691 & 0.5 & HexNAc4Hex5Fuc1 \\
\hline & & 3 & 912.7265 & 912.7255 & -1.1 & HexNAc5Hex3Fuc1 \\
\hline & & 3 & 966.7441 & 966.7424 & -1.8 & HexNAc5Hex4Fuc1 \\
\hline & & 3 & 1020.7617 & 1020.7609 & -0.8 & HexNAc5Hex5Fuc1 \\
\hline & & 3 & 1142.4724 & 1142.4657 & -5.9 & HexNAc6Hex6Fuc1 \\
\hline & & 3 & 1196.4900 & 1196.4874 & -2.2 & HexNAc6Hex7Fuc1 \\
\hline & & 2 & 1400.0783 & 1400.0753 & -2.1 & HexNAc3Hex5NeuAc1 \\
\hline & & 3 & 987.7389 & 987.7369 & -2.0 & HexNAc3Hex6NeuAc1 \\
\hline & & 3 & 1001.4144 & 1001.4117 & -2.7 & HexNAc4Hex5NeuAc1 \\
\hline & & 3 & 982.4072 & 982.4036 & -3.7 & HexNAc3Hex5Fuc1NeuAc1 \\
\hline & & 3 & 1036.4248 & 1036.4254 & 0.6 & HexNAc3Hex6Fuc1NeuAc1 \\
\hline & & 3 & 1050.1004 & 1050.0975 & -2.8 & HexNAc4Hex5Fuc1NeuAc1 \\
\hline & & 3 & 1171.8111 & 1171.8109 & -0.2 & HexNAc5Hex6Fuc1NeuAc1 \\
\hline \multirow{11}{*}{$\mathrm{C} 4$} & \multirow{11}{*}{ CSSNITGLLLTR } & 3 & 743.0138 & 743.0131 & -0.9 & HexNAc2Hex3 \\
\hline & & 3 & 797.0314 & 797.0285 & -3.6 & HexNAc2Hex 4 \\
\hline & & 3 & 851.0490 & 851.0487 & -0.4 & HexNAc2Hex 5 \\
\hline & & 3 & 905.0666 & 905.0651 & -1.7 & HexNAc2Hex6 \\
\hline & & 3 & 959.0842 & 959.0875 & 3.4 & HexNAc2Hex7 \\
\hline & & 3 & 1013.1018 & 1013.1027 & 0.9 & HexNAc2Hex8 \\
\hline & & 3 & 1067.1194 & 1067.1183 & -1.0 & HexNAc2Hex9 \\
\hline & & 2 & 1377.6096 & 1377.6044 & -3.8 & HexNAc3Hex5 \\
\hline & & 3 & 972.7597 & 972.7576 & -2.2 & HexNAc3Hex6 \\
\hline & & 3 & 986.4353 & 986.4351 & -0.2 & HexNAc4Hex5 \\
\hline & & 3 & 1040.4529 & 1040.4513 & -1.5 & HexNAc4Hex6 \\
\hline
\end{tabular}




\begin{tabular}{|c|c|c|c|c|c|c|}
\hline Domain & Peptide sequence & $\begin{array}{c}\text { Charge } \\
\text { state }\end{array}$ & $\begin{array}{c}\text { Theoretical } \\
m / z\end{array}$ & $\begin{array}{c}\text { Observed } \\
m / z\end{array}$ & $\begin{array}{c}\text { Mass } \\
\text { accurac } \\
\text { y (ppm) }\end{array}$ & Glycoform \\
\hline & & 3 & 1054.1284 & 1054.1276 & -0.8 & HexNAc5Hex5 \\
\hline & & 3 & 1175.8391 & 1175.8439 & 4.1 & HexNAc6Hex6 \\
\hline & & 3 & 981.1036 & 981.1007 & -3.0 & HexNAc4Hex4Fuc1 \\
\hline & & 3 & 1035.1212 & 1035.1204 & -0.8 & HexNAc4Hex5Fuc1 \\
\hline & & 3 & 1224.5251 & 1224.5198 & -4.3 & HexNAc6Hex6Fuc1 \\
\hline & & 3 & 1015.7739 & 1015.7717 & -2.2 & HexNAc3Hex5NeuAc1 \\
\hline & & 3 & 1069.7915 & 1069.7900 & -1.4 & HexNAc3Hex6NeuAc1 \\
\hline \multirow{10}{*}{ V5 } & \multirow{10}{*}{ DGGPEDNKTEVFR } & 3 & 1078.1100 & 1078.1046 & -5.0 & HexNAc4Hex5Fuc1 \\
\hline & & 3 & 1091.7856 & 1091.7874 & 1.6 & HexNAc5Hex4Fuc1 \\
\hline & & 3 & 1145.8032 & 1145.8070 & 3.3 & HexNAc5Hex5Fuc1 \\
\hline & & 3 & 1321.5315 & 1321.5280 & -2.6 & HexNAc6Hex7Fuc1 \\
\hline & & 3 & 1175.1418 & 1175.1448 & 2.6 & HexNAc4Hex5Fuc1NeuAc1 \\
\hline & & 3 & 1296.8526 & 1296.8560 & 2.6 & HexNAc5Hex6Fuc1NeuAc1 \\
\hline & & 3 & 1310.5281 & 1310.5313 & 2.4 & HexNAc6Hex5Fuc1NeuAc1 \\
\hline & & 3 & 1418.5633 & 1418.5696 & 4.4 & HexNAc6Hex7Fuc1NeuAc1 \\
\hline & & 3 & 1250.8387 & 1250.8345 & -3.4 & HexNAc6Hex3NeuAc2 \\
\hline & & 3 & 1515.5951 & 1515.5952 & 0.1 & HexNAc6Hex7Fuc1NeuAc2 \\
\hline
\end{tabular}


Table S2. List of identified glycopeptides associated with IIIB gp120.

\begin{tabular}{|c|c|c|c|c|c|c|}
\hline Domain & $\begin{array}{c}\text { Peptide } \\
\text { sequence }\end{array}$ & $\begin{array}{l}\text { Charge } \\
\text { state }\end{array}$ & $\begin{array}{c}\text { Theoretical } \\
\mathbf{m} / \mathbf{z}\end{array}$ & $\begin{array}{c}\text { Observed } \\
\mathbf{m} / \mathbf{z}\end{array}$ & $\begin{array}{c}\text { Mass } \\
\text { accuracy } \\
(\mathbf{p p m})\end{array}$ & Glycoform \\
\hline \multirow{38}{*}{$\mathrm{C} 1$} & \multirow{38}{*}{$\begin{array}{c}\text { VVLVNVT } \\
\text { E }\end{array}$} & 2 & 874.9192 & 874.9203 & 1.3 & HexNAc2Hex2Fuc1 \\
\hline & & 2 & 882.9166 & 882.9165 & -0.1 & HexNAc2Hex3 \\
\hline & & 2 & 963.9430 & 963.9438 & 0.8 & HexNAc2Hex4 \\
\hline & & 2 & 1044.9694 & 1044.9697 & 0.3 & HexNAc2Hex5 \\
\hline & & 2 & 1125.9958 & 1125.9940 & -1.6 & HexNAc2Hex6 \\
\hline & & 2 & 1207.0222 & 1207.0199 & -1.9 & HexNAc2Hex7 \\
\hline & & 2 & 1288.0486 & 1288.0380 & -8.2 & HexNAc2Hex8 \\
\hline & & 2 & 984.4563 & 984.4561 & -0.2 & HexNAc3Hex3 \\
\hline & & 2 & 1065.4827 & 1065.4825 & -0.2 & HexNAc3Hex4 \\
\hline & & 2 & 1146.5091 & 1146.5101 & 0.9 & HexNAc3Hex 5 \\
\hline & & 2 & 1085.9960 & 1085.9974 & 1.3 & HexNAc4Hex3 \\
\hline & & 2 & 1167.0224 & 1167.0244 & 1.7 & HexNAc4Hex4 \\
\hline & & 3 & 1248.0488 & 1248.0470 & -1.4 & HexNAc4Hex 5 \\
\hline & & 2 & 955.9456 & 955.9453 & -0.3 & HexNAc2Hex3Fuc1 \\
\hline & & 2 & 976.4589 & 976.4609 & 2.0 & HexNAc3Hex2Fuc1 \\
\hline & & 2 & 1057.4853 & 1057.4880 & 2.6 & HexNAc3Hex3Fuc1 \\
\hline & & 2 & 1138.5117 & 1138.5109 & -0.7 & HexNAc3Hex4Fuc1 \\
\hline & & 2 & 1219.5381 & 1219.5402 & 1.7 & HexNAc3Hex5Fuc1 \\
\hline & & 3 & 773.0191 & 773.0183 & -1.0 & HexNAc4Hex3Fuc1 \\
\hline & & 3 & 827.0367 & 827.0386 & 2.3 & HexNAc4Hex4Fuc1 \\
\hline & & 3 & 881.0543 & 881.0545 & 0.2 & HexNAc4Hex5Fuc1 \\
\hline & & 3 & 935.0719 & 935.0718 & -0.1 & HexNAc4Hex6Fuc1 \\
\hline & & 3 & 840.7122 & 840.7115 & -0.8 & HexNAc5Hex3Fuc1 \\
\hline & & 3 & 894.7298 & 894.7305 & 0.8 & HexNAc5Hex4Fuc1 \\
\hline & & 3 & 948.7474 & 948.7469 & -0.5 & HexNAc5Hex5Fuc1 \\
\hline & & 3 & 1002.7650 & 1002.7620 & -3.0 & HexNAc5Hex6Fuc1 \\
\hline & & 3 & 1070.4581 & 1070.4573 & -0.7 & HexNAc6Hex6Fuc1 \\
\hline & & 3 & 1124.4757 & 1124.4734 & -2.0 & HexNAc6Hex7Fuc1 \\
\hline & & 3 & 929.7402 & 929.7396 & -0.6 & HexNAc4Hex5Fuc2 \\
\hline & & 3 & 1051.1108 & 1051.1060 & -4.6 & HexNAc5Hex6NeuAc1 \\
\hline & & 3 & 1105.1284 & 1105.1280 & -0.4 & HexNAc5Hex7NeuAc1 \\
\hline & & 3 & 1226.8392 & 1226.8348 & -3.6 & HexNAc6Hex8NeuAc1 \\
\hline & & 2 & 1203.0330 & 1203.0320 & -0.8 & HexNAc3Hex3Fuc1NeuAc1 \\
\hline & & 2 & 1284.0594 & 1284.0592 & -0.2 & HexNAc3Hex4Fuc1NeuAc1 \\
\hline & & 2 & 1365.0858 & 1365.0828 & -2.2 & HexNAc3Hex5Fuc1NeuAc1 \\
\hline & & 3 & 924.0685 & 924.0678 & -0.8 & HexNAc4Hex4Fuc1NeuAc1 \\
\hline & & 3 & 978.0861 & 978.0866 & 0.5 & HexNAc4Hex5Fuc1NeuAc1 \\
\hline & & 3 & 1032.1037 & 1032.1033 & -0.4 & HexNAc4Hex6Fuc1NeuAc1 \\
\hline
\end{tabular}




\begin{tabular}{|c|c|c|c|c|c|c|}
\hline Domain & $\begin{array}{c}\text { Peptide } \\
\text { sequence }\end{array}$ & $\begin{array}{c}\text { Charge } \\
\text { state }\end{array}$ & $\begin{array}{c}\text { Theoretical } \\
\mathbf{m} / \mathbf{z}\end{array}$ & $\begin{array}{c}\text { Observed } \\
\mathbf{m} / \mathbf{z}\end{array}$ & $\begin{array}{c}\text { Mass } \\
\text { accuracy } \\
(\mathrm{ppm})\end{array}$ & Glycoform \\
\hline & & 3 & 1045.7792 & 1045.7780 & -1.1 & HexNAc5Hex5Fuc1NeuAc1 \\
\hline & & 3 & 1099.7968 & 1099.7960 & -0.7 & HexNAc5Hex6Fuc1NeuAc1 \\
\hline & & 3 & 1153.8144 & 1153.8162 & 1.6 & HexNAc5Hex7Fuc1NeuAc1 \\
\hline & & 3 & 1167.4899 & 1167.4889 & -0.9 & HexNAc6Hex6Fuc1NeuAc1 \\
\hline & & 3 & 1221.5075 & 1221.5073 & -0.2 & HexNAc6Hex7Fuc1NeuAc1 \\
\hline & & 3 & 1343.2183 & 1343.2225 & 3.1 & HexNAc7Hex8Fuc1NeuAc1 \\
\hline & & 3 & 1026.7720 & 1026.7720 & 0.0 & HexNAc4Hex5Fuc2NeuAc1 \\
\hline & & 3 & 1148.4828 & 1148.4800 & -2.4 & HexNAc5Hex6Fuc2NeuAc1 \\
\hline & & 3 & 1080.4495 & 1080.4490 & -0.5 & HexNAc4Hex6NeuAc2 \\
\hline & & 3 & 1202.1602 & 1202.1605 & 0.2 & HexNAc5Hex7NeuAc2 \\
\hline & & 3 & 1323.8710 & 1323.8645 & -4.9 & HexNAc6Hex8NeuAc2 \\
\hline & & 3 & 1075.1179 & 1075.1169 & -0.9 & HexNAc4Hex5Fuc1NeuAc2 \\
\hline & & 3 & 1142.8110 & 1142.8136 & 2.3 & HexNAc5Hex5Fuc1NeuAc2 \\
\hline & & 3 & 1196.8286 & 1196.8275 & -0.9 & HexNAc5Hex6Fuc1NeuAc2 \\
\hline & & 3 & 1264.5217 & 1264.5251 & 2.7 & HexNAc6Hex6Fuc1NeuAc2 \\
\hline & & 3 & 1318.5393 & 1318.5374 & -1.4 & HexNAc6Hex7Fuc1NeuAc2 \\
\hline & & 3 & 1123.8038 & 1123.8036 & -0.2 & HexNAc4Hex5Fuc2NeuAc2 \\
\hline \multirow{26}{*}{$\mathrm{V} 1 / \mathrm{V} 2$} & \multirow{26}{*}{$\underset{\text { SIR }}{\underline{\text { NCSFNIST }}}$} & 3 & 839.0177 & 839.0173 & -0.5 & HexNAc2Hex 5 \\
\hline & & 3 & 1136.4568 & 1136.4561 & -0.6 & HexNAc4Hex 8 \\
\hline & & 3 & 1190.4744 & 1190.4670 & -6.2 & HexNAc4Hex9 \\
\hline & & 3 & 1244.4920 & 1244.4907 & -1.0 & HexNAc4Hex 10 \\
\hline & & 3 & 1298.5096 & 1298.5084 & -0.9 & HexNAc4Hex11 \\
\hline & & 3 & 1352.5272 & 1352.5255 & -1.3 & HexNAc4Hex12 \\
\hline & & 3 & 1406.5448 & 1406.5494 & 3.3 & HexNAc4Hex13 \\
\hline & & 3 & 1204.1499 & 1204.1509 & 0.8 & HexNAc5Hex8 \\
\hline & & 3 & 915.0547 & 915.0521 & -2.8 & HexNAc4Hex3Fuc1 \\
\hline & & 3 & 969.0723 & 969.0701 & -2.3 & HexNAc4Hex4Fuc1 \\
\hline & & 3 & 1023.0899 & 1023.0886 & -1.3 & HexNAc4Hex5Fuc1 \\
\hline & & 3 & 1185.1427 & 1185.1437 & 0.8 & HexNAc4Hex8Fuc1 \\
\hline & & 3 & 1144.8007 & 1144.7991 & -1.4 & HexNAc5Hex6Fuc1 \\
\hline & & 3 & 1252.8359 & 1252.8356 & -0.2 & HexNAc5Hex8Fuc1 \\
\hline & & 4 & 950.1354 & 950.1316 & -4.0 & HexNAc6Hex7Fuc1 \\
\hline & & 3 & 1320.5290 & 1320.5284 & -0.5 & HexNAc6Hex8Fuc1 \\
\hline & & 4 & 1071.6750 & 1071.6818 & 6.3 & HexNAc6Hex10Fuc1 \\
\hline & & 4 & 1122.4448 & 1122.4432 & -1.4 & HexNAc7Hex10Fuc1 \\
\hline & & 4 & 1162.9580 & 1162.9569 & -0.9 & HexNAc7Hex11Fuc1 \\
\hline & & 4 & 1092.1883 & 1092.1854 & -2.7 & HexNAc8Hex8Fuc1 \\
\hline & & 4 & 1132.7015 & 1132.6975 & -3.5 & HexNAc8Hex9Fuc1 \\
\hline & & 4 & 1173.2147 & 1173.2142 & -0.4 & HexNAc8Hex10Fuc1 \\
\hline & & 4 & 1264.4977 & 1264.4967 & -0.8 & HexNAc9Hex11Fuc1 \\
\hline & & 4 & 1118.4461 & 1118.4463 & 0.2 & HexNAc7Hex9Fuc2 \\
\hline & & 4 & 1128.7027 & 1128.7004 & -2.0 & HexNAc8Hex8Fuc2 \\
\hline & & 4 & 1169.2159 & 1169.2142 & -1.5 & HexNAc8Hex9Fuc2 \\
\hline
\end{tabular}




\begin{tabular}{|c|c|c|c|c|c|c|}
\hline Domain & $\begin{array}{c}\text { Peptide } \\
\text { sequence }\end{array}$ & $\begin{array}{l}\text { Charge } \\
\text { state }\end{array}$ & $\begin{array}{c}\text { Theoretical } \\
\mathbf{m} / \mathbf{z}\end{array}$ & $\begin{array}{c}\text { Observed } \\
\mathbf{m} / \mathbf{z}\end{array}$ & $\begin{array}{c}\text { Mass } \\
\text { accuracy } \\
(\mathrm{ppm})\end{array}$ & Glycoform \\
\hline & & 4 & 1209.7291 & 1209.7277 & -1.2 & HexNAc8Hex10Fuc2 \\
\hline & & 4 & 1219.9858 & 1219.9889 & 2.5 & HexNAc9Hex9Fuc2 \\
\hline & & 4 & 1260.4990 & 1260.4945 & -3.6 & HexNAc9Hex10Fuc2 \\
\hline & & 4 & 1301.0122 & 1301.0087 & -2.7 & HexNAc9Hex11Fuc2 \\
\hline & & 3 & 1071.4358 & 1071.4360 & 0.2 & HexNAc4Hex5NeuAc1 \\
\hline & & 3 & 1066.1041 & 1066.1011 & -2.8 & HexNAc4Hex4Fuc1NeuAc1 \\
\hline & & 3 & 1120.1217 & 1120.1200 & -1.5 & HexNAc4Hex5Fuc1NeuAc1 \\
\hline & & 3 & 1241.8325 & 1241.8325 & 0.0 & HexNAc5Hex6Fuc1NeuAc1 \\
\hline & & 4 & 1022.9092 & 1022.9042 & -4.9 & HexNAc6Hex7Fuc1NeuAc1 \\
\hline & & 3 & 1417.5608 & 1417.5618 & 0.7 & HexNAc6Hex8Fuc1NeuAc1 \\
\hline & & 4 & 1144.4488 & 1144.4474 & -1.2 & HexNAc6Hex10Fuc1NeuAc1 \\
\hline & & 4 & 1195.2187 & 1195.2137 & -4.2 & HexNAc7Hex10Fuc1NeuAc1 \\
\hline & & 4 & 1235.7319 & 1235.7362 & 3.5 & HexNAc7Hex11Fuc1NeuAc1 \\
\hline & & 4 & 1018.9105 & 1018.9057 & -4.7 & HexNAc6Hex6Fuc2NeuAc1 \\
\hline & & 4 & 1059.4237 & 1059.4207 & -2.8 & HexNAc6Hex7Fuc2NeuAc1 \\
\hline & & 4 & 1110.1935 & 1110.1887 & -4.3 & HexNAc7Hex7Fuc2NeuAc1 \\
\hline & & 4 & 1191.2199 & 1191.2172 & -2.3 & HexNAc7Hex9Fuc2NeuAc1 \\
\hline & & 4 & 1055.4250 & 1055.4224 & -2.5 & HexNAc6Hex9Fuc3NeuAc1 \\
\hline & & 4 & 1308.5057 & 1308.5066 & 0.7 & HexNAc7Hex11Fuc1NeuAc2 \\
\hline & & 4 & 1263.9938 & 1263.9904 & -2.7 & HexNAc7Hex9Fuc2NeuAc2 \\
\hline & & 4 & 1304.5070 & 1304.5078 & 0.6 & HexNAc7Hex10Fuc2NeuAc2 \\
\hline & & 3 & 1374.5466 & 1374.5450 & -1.2 & HexNAc6Hex9Fuc1 \\
\hline \multirow{8}{*}{$\mathrm{V} 2 / \mathrm{C} 2$} & \multirow{8}{*}{$\begin{array}{c}\text { LDIIPIDND } \\
\text { TTSYTLTS } \\
\text { CNTSVITQ } \\
\text { ACPK }\end{array}$} & 4 & 1378.8437 & 1378.8450 & 0.9 & HexNAc4Hex9 \\
\hline & & 4 & 1419.3569 & 1419.3563 & -0.4 & HexNAc4Hex10 \\
\hline & & 4 & 1459.8701 & 1459.8769 & 4.7 & HexNAc4Hex11 \\
\hline & & 4 & 1500.3833 & 1500.3743 & -6.0 & HexNAc4Hex12 \\
\hline & & 4 & 1425.6148 & 1425.6210 & 4.3 & HexNAc5Hex8Fuc1 \\
\hline & & 4 & 1476.3847 & 1476.3740 & -7.2 & HexNAc6Hex8Fuc1 \\
\hline & & 4 & 1567.6677 & 1567.6700 & 1.5 & HexNAc7Hex9Fuc1 \\
\hline & & 4 & 1533.4125 & 1533.4176 & 3.3 & HexNAc8Hex6Fuc2 \\
\hline \multirow{13}{*}{$\mathrm{C} 2$} & \multirow{13}{*}{$\begin{array}{l}\text { TFNGTGP } \\
\text { CTNVSTV } \\
\text { QCTHGIR }\end{array}$} & 4 & 1023.6792 & 1023.6802 & 1.0 & HexNAc4Hex6 \\
\hline & & 4 & 1064.1924 & 1064.1975 & 4.8 & HexNAc4Hex7 \\
\hline & & 4 & 1104.7056 & 1104.7032 & -2.2 & HexNAc4Hex 8 \\
\hline & & 4 & 1145.2188 & 1145.2220 & 2.8 & HexNAc4Hex9 \\
\hline & & 4 & 1185.7320 & 1185.7288 & -2.7 & HexNAc4Hex 10 \\
\hline & & 4 & 1226.2452 & 1226.2418 & -2.8 & HexNAc4Hex11 \\
\hline & & 4 & 1266.7584 & 1266.7540 & -3.5 & HexNAc4Hex12 \\
\hline & & 4 & 1307.2716 & 1307.2672 & -3.4 & HexNAc4Hex13 \\
\hline & & 4 & 1347.7848 & 1347.7788 & -4.5 & HexNAc4Hex14 \\
\hline & & 4 & 1388.2980 & 1388.2943 & -2.7 & HexNAc4Hex15 \\
\hline & & 4 & 1428.8112 & 1428.8062 & -3.5 & HexNAc4Hex 16 \\
\hline & & 4 & 1469.3244 & 1469.3206 & -2.6 & HexNAc4Hex17 \\
\hline & & 4 & 1236.5018 & 1236.5035 & 1.4 & HexNAc5Hex 10 \\
\hline
\end{tabular}




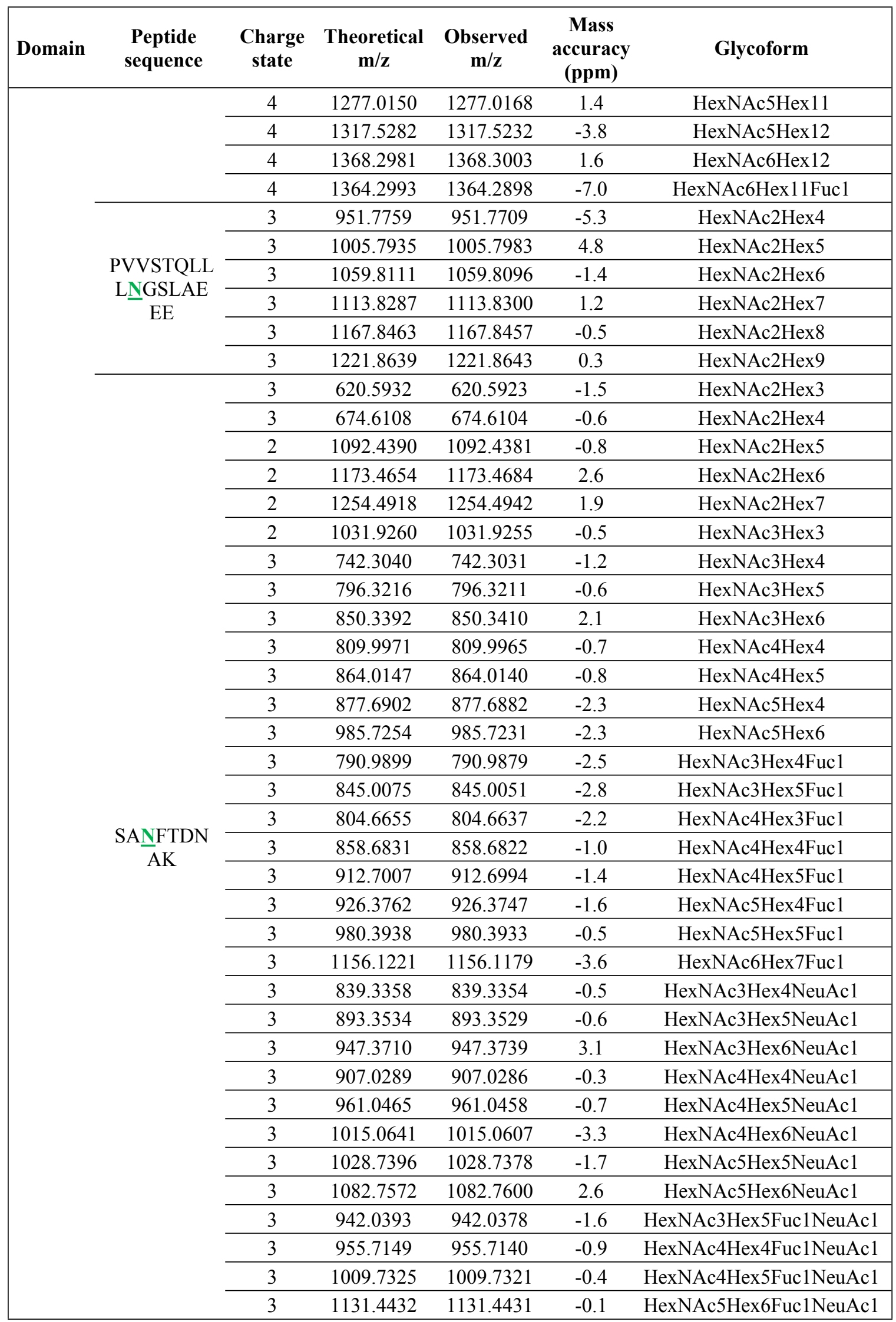




\begin{tabular}{|c|c|c|c|c|c|c|}
\hline Domain & $\begin{array}{l}\text { Peptide } \\
\text { sequence }\end{array}$ & $\begin{array}{l}\text { Charge } \\
\text { state }\end{array}$ & $\begin{array}{c}\text { Theoretical } \\
\mathbf{m} / \mathbf{z}\end{array}$ & $\begin{array}{c}\text { Observed } \\
\mathbf{m} / \mathbf{z}\end{array}$ & $\begin{array}{c}\text { Mass } \\
\text { accuracy } \\
(\mathrm{ppm})\end{array}$ & Glycoform \\
\hline & & 3 & 1253.1539 & 1253.1533 & -0.5 & HexNAc6Hex7Fuc1NeuAc1 \\
\hline & & 3 & 1058.0783 & 1058.0767 & -1.5 & HexNAc4Hex5NeuAc2 \\
\hline & & 3 & 1106.7643 & 1106.7631 & -1.1 & HexNAc4Hex5Fuc1NeuAc2 \\
\hline & & 2 & 723.8879 & 723.8879 & 0.0 & HexNAc1 \\
\hline & & 2 & 906.4540 & 906.4562 & 2.4 & HexNAc2Hex1 \\
\hline & & 3 & 712.6736 & 712.6728 & -1.1 & HexNAc2Hex3 \\
\hline & & 3 & 766.6912 & 766.6908 & -0.5 & HexNAc2Hex4 \\
\hline & & 3 & 820.7088 & 820.7081 & -0.9 & HexNAc2Hex5 \\
\hline & & 3 & 874.7264 & 874.7255 & -1.0 & HexNAc2Hex6 \\
\hline & & 3 & 928.7440 & 928.7431 & -1.0 & HexNAc2Hex 7 \\
\hline & & 3 & 982.7616 & 982.7610 & -0.6 & HexNAc2Hex8 \\
\hline & & 3 & 1036.7792 & 1036.7783 & -0.9 & HexNAc2Hex9 \\
\hline & & 3 & 888.4020 & 888.4030 & 1.1 & HexNAc3Hex5 \\
\hline & & 3 & 942.4196 & 942.4188 & -0.8 & HexNAc3Hex6 \\
\hline & & 3 & 902.0775 & 902.0783 & 0.9 & HexNAc4Hex4 \\
\hline & & 3 & 956.0951 & 956.0932 & -2.0 & HexNAc4Hex5 \\
\hline & TIIVQLNQ & 3 & 896.7459 & 896.7501 & 4.7 & HexNAc4Hex3Fuc1 \\
\hline & SV & 3 & 950.7635 & 950.7628 & -0.7 & HexNAc4Hex4Fuc1 \\
\hline & & 3 & 1004.7811 & 1004.7814 & 0.3 & HexNAc4Hex5Fuc1 \\
\hline & & 3 & 1072.4742 & 1072.4716 & -2.4 & HexNAc5Hex5Fuc1 \\
\hline & & 3 & 1126.4918 & 1126.4912 & -0.5 & HexNAc5Hex6Fuc1 \\
\hline & & 3 & 1248.2025 & 1248.2075 & 4.0 & HexNAc6Hex7Fuc1 \\
\hline & & 3 & 985.4338 & 985.4324 & -1.4 & HexNAc3Hex5NeuAc1 \\
\hline & & 3 & 1039.4514 & 1039.4553 & 3.8 & HexNAc3Hex6NeuAc1 \\
\hline & & 3 & 1053.1269 & 1053.1281 & 1.1 & HexNAc4Hex5NeuAc1 \\
\hline & & 3 & 1107.1445 & 1107.1445 & 0.0 & HexNAc4Hex6NeuAc1 \\
\hline & & 3 & 1101.8129 & 1101.8114 & -1.4 & HexNAc4Hex5Fuc1NeuAc1 \\
\hline & & 3 & 1223.5236 & 1223.5232 & -0.3 & HexNAc5Hex6Fuc1NeuAc1 \\
\hline & & 3 & 1345.2343 & 1345.2386 & 3.2 & HexNAc6Hex7Fuc1NeuAc1 \\
\hline & & 3 & 1150.1587 & 1150.1552 & -3.0 & HexNAc4Hex5NeuAc2 \\
\hline & & 3 & 1198.8447 & 1198.8414 & -2.8 & HexNAc4Hex5Fuc1NeuAc2 \\
\hline \multirow{12}{*}{$\mathrm{C} 3$} & \multirow{11}{*}{ QAHCNISR } & 2 & 1020.4202 & 1020.4248 & 4.5 & HexNAc2Hex 4 \\
\hline & & 3 & 734.6335 & 734.6354 & 2.6 & HexNAc2Hex 5 \\
\hline & & 3 & 788.6511 & 788.6530 & 2.4 & HexNAc2Hex6 \\
\hline & & 3 & 842.6687 & 842.6704 & 2.0 & HexNAc2Hex7 \\
\hline & & 3 & 896.6863 & 896.6888 & 2.8 & HexNAc2Hex 8 \\
\hline & & 3 & 950.7039 & 950.7065 & 2.7 & HexNAc2Hex9 \\
\hline & & 3 & 864.6881 & 864.6910 & 3.4 & HexNAc4Hex4Fuc1 \\
\hline & & 3 & 918.7057 & 918.7087 & 3.3 & HexNAc4Hex5Fuc1 \\
\hline & & 3 & 878.3637 & 878.3675 & 4.3 & HexNAc5Hex3Fuc1 \\
\hline & & 3 & 1015.7375 & 1015.7340 & -3.4 & HexNAc4Hex5Fuc1NeuAc1 \\
\hline & & 3 & 1137.4483 & 1137.4523 & 3.5 & HexNAc5Hex6Fuc1NeuAc1 \\
\hline & WNNTLK & 2 & 834.3671 & 834.3676 & 0.6 & HexNAc2Hex3 \\
\hline
\end{tabular}




\begin{tabular}{|c|c|c|c|c|c|c|}
\hline Domain & $\begin{array}{c}\text { Peptide } \\
\text { sequence }\end{array}$ & $\begin{array}{l}\text { Charge } \\
\text { state }\end{array}$ & $\begin{array}{c}\text { Theoretical } \\
\mathbf{m} / \mathbf{z}\end{array}$ & $\begin{array}{c}\text { Observed } \\
\mathbf{m} / \mathbf{z}\end{array}$ & $\begin{array}{c}\text { Mass } \\
\text { accuracy } \\
(\mathrm{ppm})\end{array}$ & Glycoform \\
\hline & & 2 & 915.3935 & 915.3924 & -1.2 & HexNAc2Hex4 \\
\hline & & 2 & 996.4199 & 996.4188 & -1.1 & HexNAc2Hex5 \\
\hline & & 2 & 1077.4463 & 1077.4457 & -0.6 & HexNAc2Hex6 \\
\hline & & 2 & 1158.4727 & 1158.4723 & -0.3 & HexNAc2Hex7 \\
\hline & & 2 & 1239.4991 & 1239.4982 & -0.7 & HexNAc2Hex 8 \\
\hline & & 2 & 1320.5255 & 1320.5240 & -1.1 & HexNAc2Hex9 \\
\hline & & 2 & 935.9068 & 935.9092 & 2.6 & HexNAc3Hex3 \\
\hline & & 2 & 1016.9332 & 1016.9329 & -0.3 & HexNAc3Hex4 \\
\hline & & 2 & 1097.9596 & 1097.9597 & 0.1 & HexNAc3Hex 5 \\
\hline & & 2 & 1178.9860 & 1178.9801 & -5.0 & HexNAc3Hex6 \\
\hline & & 2 & 1199.4993 & 1199.4983 & -0.8 & HexNAc4Hex5 \\
\hline & & 2 & 1272.5282 & 1272.5222 & -4.7 & HexNAc4Hex5Fuc1 \\
\hline & & 2 & 1162.4809 & 1162.4825 & 1.4 & HexNAc3Hex4NeuAc1 \\
\hline & & 2 & 1243.5073 & 1243.5052 & -1.7 & HexNAc3Hex5NeuAc1 \\
\hline & & 2 & 1324.5337 & 1324.5389 & 3.9 & HexNAc3Hex6NeuAc1 \\
\hline & & 3 & 945.7197 & 945.7181 & -1.7 & HexNAc4Hex5Fuc1NeuAc1 \\
\hline & \multirow{27}{*}{$\begin{array}{c}\text { LREQFGN } \\
\text { NK(T) }\end{array}$} & 3 & 774.6707 & 774.6710 & 0.4 & HexNAc2Hex5 \\
\hline & & 3 & 828.6883 & 828.6924 & 4.9 & HexNAc2Hex6 \\
\hline & & 3 & 842.3639 & 842.3627 & -1.4 & HexNAc3Hex5 \\
\hline & & 3 & 896.3815 & 896.3802 & -1.5 & HexNAc3Hex6 \\
\hline & & 3 & 856.0394 & 856.0423 & 3.4 & HexNAc4Hex4 \\
\hline & & 3 & 910.0570 & 910.0567 & -0.3 & HexNAc4Hex 5 \\
\hline & & 3 & 904.7254 & 904.7256 & 0.2 & HexNAc4Hex4Fuc1 \\
\hline & & 3 & 958.7430 & 958.7422 & -0.8 & HexNAc4Hex5Fuc1 \\
\hline & & 3 & 1026.4361 & 1026.4358 & -0.3 & HexNAc5Hex5Fuc1 \\
\hline & & 3 & 1080.4537 & 1080.4529 & -0.7 & HexNAc5Hex6Fuc1 \\
\hline & & 3 & 1148.1468 & 1148.1508 & 3.5 & HexNAc6Hex6Fuc1 \\
\hline & & 3 & 1202.1644 & 1202.1591 & -4.4 & HexNAc6Hex7Fuc1 \\
\hline & & 3 & 885.3781 & 885.3782 & 0.1 & HexNAc3Hex4NeuAc1 \\
\hline & & 3 & 939.3957 & 939.3952 & -0.5 & HexNAc3Hex5NeuAc1 \\
\hline & & 3 & 993.4133 & 993.4117 & -1.6 & HexNAc3Hex6NeuAc1 \\
\hline & & 3 & 953.0712 & 953.0731 & 2.0 & HexNAc4Hex4NeuAc1 \\
\hline & & 3 & 1007.0888 & 1007.0875 & -1.3 & HexNAc4Hex5NeuAc1 \\
\hline & & 3 & 1061.1064 & 1061.1068 & 0.4 & HexNAc4Hex6NeuAc1 \\
\hline & & 3 & 934.0640 & 934.0626 & -1.5 & HexNAc3Hex4Fuc1NeuAc1 \\
\hline & & 3 & 988.0816 & 988.0819 & 0.3 & HexNAc3Hex5Fuc1NeuAc1 \\
\hline & & 3 & 1042.0992 & 1042.0920 & -6.9 & HexNAc3Hex6Fuc1NeuAc1 \\
\hline & & 3 & 1001.7572 & 1001.7559 & -1.3 & HexNAc4Hex4Fuc1NeuAc1 \\
\hline & & 3 & 1055.7748 & 1055.7737 & -1.0 & HexNAc4Hex5Fuc1NeuAc1 \\
\hline & & 3 & 1069.4503 & 1069.4498 & -0.5 & HexNAc5Hex4Fuc1NeuAc1 \\
\hline & & 3 & 1123.4679 & 1123.4633 & -4.1 & HexNAc5Hex5Fuc1NeuAc1 \\
\hline & & 3 & 1177.4855 & 1177.4880 & 2.1 & HexNAc5Hex6Fuc1NeuAc1 \\
\hline & & 3 & 1299.1962 & 1299.1962 & 0.0 & HexNAc6Hex7Fuc1NeuAc1 \\
\hline
\end{tabular}




\begin{tabular}{|c|c|c|c|c|c|c|}
\hline Domain & $\begin{array}{l}\text { Peptide } \\
\text { sequence }\end{array}$ & $\begin{array}{l}\text { Charge } \\
\text { state }\end{array}$ & $\begin{array}{l}\text { Theoretical } \\
\mathbf{m} / \mathbf{z}\end{array}$ & $\begin{array}{c}\text { Observed } \\
\mathbf{m} / \mathbf{z}\end{array}$ & $\begin{array}{c}\text { Mass } \\
\text { accuracy } \\
(\text { ppm })\end{array}$ & Glycoform \\
\hline & & 3 & 1158.1382 & 1158.1393 & 0.9 & $\begin{array}{c}\text { HexNAc4Hex5Fuc1NeuAc1Ne } \\
\text { uGc1 }\end{array}$ \\
\hline & & 3 & 1152.8066 & 1152.8052 & -1.2 & HexNAc4Hex5Fuc1NeuAc2 \\
\hline & & 3 & 1220.4997 & 1220.5016 & 1.6 & HexNAc5Hex5Fuc1NeuAc2 \\
\hline & & 3 & 1274.5173 & 1274.5206 & 2.6 & HexNAc5Hex6Fuc1NeuAc2 \\
\hline & & 3 & 1396.2280 & 1396.2300 & 1.4 & HexNAc6Hex7Fuc1NeuAc2 \\
\hline \multirow{35}{*}{ V4 } & \multirow{35}{*}{$\begin{array}{c}\text { GSNNTEG } \\
\text { SDTITLPC } \\
\mathrm{R}\end{array}$} & 3 & 926.0535 & 926.0501 & -3.7 & HexNAc2Hex4 \\
\hline & & 3 & 980.0711 & 980.0698 & -1.3 & HexNAc2Hex5 \\
\hline & & 3 & 1034.0887 & 1034.0896 & 0.9 & HexNAc2Hex6 \\
\hline & & 3 & 1088.1063 & 1088.1068 & 0.5 & HexNAc2Hex7 \\
\hline & & 3 & 939.7290 & 939.7308 & 1.9 & HexNAc3Hex3 \\
\hline & & 3 & 993.7466 & 993.7433 & -3.3 & HexNAc3Hex 4 \\
\hline & & 3 & 1101.7818 & 1101.7819 & 0.1 & HexNAc3Hex6 \\
\hline & & 3 & 1007.4221 & 1007.4220 & -0.1 & HexNAc4Hex3 \\
\hline & & 3 & 1115.4573 & 1115.4574 & 0.1 & HexNAc4Hex5 \\
\hline & & 3 & 988.4150 & 988.4135 & -1.5 & HexNAc3Hex3Fuc1 \\
\hline & & 3 & 1042.4326 & 1042.4312 & -1.3 & HexNAc3Hex4Fuc1 \\
\hline & & 3 & 1096.4502 & 1096.4512 & 0.9 & HexNAc3Hex5Fuc1 \\
\hline & & 3 & 1056.1081 & 1056.1066 & -1.4 & HexNAc4Hex3Fuc1 \\
\hline & & 3 & 1110.1257 & 1110.1238 & -1.7 & HexNAc4Hex4Fuc1 \\
\hline & & 3 & 1164.1433 & 1164.1407 & -2.2 & HexNAc4Hex5Fuc1 \\
\hline & & 3 & 1218.1609 & 1218.1571 & -3.1 & HexNAc4Hex6Fuc1 \\
\hline & & 3 & 1231.8364 & 1231.8413 & 4.0 & HexNAc5Hex5Fuc1 \\
\hline & & 3 & 1285.8540 & 1285.8517 & -1.8 & HexNAc5Hex6Fuc1 \\
\hline & & 3 & 1144.7960 & 1144.7968 & 0.7 & HexNAc3Hex5NeuAc1 \\
\hline & & 3 & 1212.4891 & 1212.4850 & -3.4 & $\begin{array}{c}\text { HexNAc4Hex5NeuAc1/HexN } \\
\text { Ac4Hex4Fuc1NeuGc1 }{ }^{a}\end{array}$ \\
\hline & & 3 & 1266.5067 & 1266.5038 & -2.3 & HexNAc4Hex5fuc1NeuGc1 \\
\hline & & 3 & 1407.5648 & 1407.5625 & -1.6 & HexNAc6Hex7Fuc1 \\
\hline & & 3 & 1139.4644 & 1139.4617 & -2.4 & HexNAc3Hex4Fuc1NeuAc1 \\
\hline & & 3 & 1207.1575 & 1207.1553 & -1.8 & HexNAc4Hex4Fuc1NeuAc1 \\
\hline & & 3 & 1261.1751 & 1261.1719 & -2.5 & HexNAc4Hex5Fuc1NeuAc1 \\
\hline & & 3 & 1328.8682 & 1328.8658 & -1.8 & HexNAc5Hex5Fuc1NeuAc1 \\
\hline & & 3 & 1382.8858 & 1382.8827 & -2.2 & HexNAc5Hex6Fuc1NeuAc1 \\
\hline & & 3 & 1504.5966 & 1504.5970 & 0.3 & HexNAc6Hex7Fuc1NeuAc1 \\
\hline & & 3 & 1363.5385 & 1363.5348 & -2.7 & HexNAc4Hex6NeuAc2 \\
\hline & & 3 & 1358.2069 & 1358.2040 & -2.1 & HexNAc4Hex5Fuc1NeuAc2 \\
\hline & & 3 & 1479.9176 & 1479.9146 & -2.0 & HexNAc5Hex6Fuc1NeuAc2 \\
\hline & & 4 & 1201.4731 & 1201.4709 & -1.8 & HexNAc6Hex7Fuc1NeuAc2 \\
\hline & & 3 & 1406.8929 & 1406.8904 & -1.8 & HexNAc4Hex5Fuc2NeuAc2 \\
\hline & & 3 & 1576.9494 & 1576.9443 & -3.2 & HexNAc5Hex6Fuc1NeuAc3 \\
\hline & & 4 & 1274.2469 & 1274.2437 & -2.5 & HexNAc6Hex7Fuc1NeuAc3 \\
\hline $\mathrm{C} 4$ & CSSNITGL & 2 & 1195.0435 & 1195.0433 & -0.2 & HexNAc2Hex4 \\
\hline
\end{tabular}




\begin{tabular}{|c|c|c|c|c|c|c|}
\hline Domain & $\begin{array}{c}\text { Peptide } \\
\text { sequence }\end{array}$ & $\begin{array}{c}\text { Charge } \\
\text { state }\end{array}$ & $\begin{array}{c}\text { Theoretical } \\
\mathbf{m} / \mathbf{z}\end{array}$ & $\begin{array}{c}\text { Observed } \\
\mathbf{m} / \mathbf{z}\end{array}$ & $\begin{array}{c}\text { Mass } \\
\text { accuracy } \\
(\mathrm{ppm})\end{array}$ & Glycoform \\
\hline & \multirow[t]{21}{*}{$\overline{\text { LLTR }}$} & 2 & 1276.0699 & 1276.0676 & -1.8 & HexNAc2Hex5 \\
\hline & & 2 & 1357.0963 & 1357.0948 & -1.1 & HexNAc2Hex6 \\
\hline & & 2 & 1438.1227 & 1438.1234 & 0.5 & HexNAc2Hex7 \\
\hline & & 2 & 1519.1491 & 1519.1503 & 0.8 & HexNAc2Hex8 \\
\hline & & 2 & 1600.1755 & 1600.1777 & 1.4 & HexNAc2Hex9 \\
\hline & & 2 & 1215.5568 & 1215.5583 & 1.2 & HexNAc3Hex3 \\
\hline & & 2 & 1296.5832 & 1296.5812 & -1.5 & HexNAc3Hex4 \\
\hline & & 3 & 918.7421 & 918.7469 & 5.2 & HexNAc3Hex 5 \\
\hline & & 3 & 972.7597 & 972.7557 & -4.1 & HexNAc3Hex6 \\
\hline & & 3 & 986.4353 & 986.4349 & -0.4 & HexNAc4Hex 5 \\
\hline & & 3 & 927.0860 & 927.0880 & 2.2 & HexNAc4Hex3Fuc1 \\
\hline & & 3 & 981.1036 & 981.1048 & 1.2 & HexNAc4Hex4Fuc1 \\
\hline & & 3 & 1035.1212 & 1035.1234 & 2.1 & HexNAc4Hex5Fuc1 \\
\hline & & 3 & 1156.8320 & 1156.8336 & 1.4 & HexNAc5Hex6Fuc1 \\
\hline & & 3 & 1278.5427 & 1278.5435 & 0.6 & HexNAc6Hex7Fuc1 \\
\hline & & 3 & 1015.7739 & 1015.7736 & -0.3 & HexNAc3Hex5NeuAc1 \\
\hline & & 3 & 1069.7915 & 1069.7974 & 5.5 & HexNAc3Hex6NeuAc1 \\
\hline & & 3 & 1083.4671 & 1083.4677 & 0.6 & HexNAc4Hex5NeuAc1 \\
\hline & & 3 & 1078.1354 & 1078.1360 & 0.6 & HexNAc4Hex4Fuc1NeuAc1 \\
\hline & & 3 & 1132.1530 & 1132.1532 & 0.2 & HexNAc4Hex5Fuc1NeuAc1 \\
\hline & & 3 & 1253.8638 & 1253.8617 & -1.7 & HexNAc5Hex6Fuc1NeuAc1 \\
\hline \multirow{4}{*}{ V5 } & \multirow{4}{*}{$\begin{array}{c}\text { DGGNSNN } \\
\text { ESEIFR }\end{array}$} & 3 & 885.6855 & 885.6835 & -2.3 & HexNAc2Hex5 \\
\hline & & 3 & 1015.7402 & 1015.7411 & 0.9 & HexNAc4Hex4Fuc1 \\
\hline & & 3 & 1069.7578 & 1069.7610 & 3.0 & HexNAc4Hex5Fuc1 \\
\hline & & 3 & 1166.7896 & 1166.7925 & 2.5 & HexNAc4Hex5Fuc1NeuAc1 \\
\hline
\end{tabular}

a. The $m / z$ value 1212.4891 was assigned to two glycoforms, HexNAc4Hex5NeuAc1 and HexNAc4Hex4Fuc1NeuGc1, because this $m / z$ matched the theoretical values of both glycoforms and $\mathrm{MS} / \mathrm{MS}$ also proved their existence. 
Table S3. List of identified glycopeptides associated with 96ZM651 gp120.

\begin{tabular}{|c|c|c|c|c|c|c|}
\hline Domain & $\begin{array}{c}\text { Peptide } \\
\text { sequence }\end{array}$ & $\begin{array}{c}\text { Charge } \\
\text { state }\end{array}$ & $\begin{array}{c}\text { Theoretical } \\
\mathbf{m} / \mathbf{z}\end{array}$ & $\begin{array}{c}\text { Observed } \\
\mathbf{m} / \mathbf{z}\end{array}$ & $\begin{array}{c}\text { Mass } \\
\text { accuracy } \\
\text { (ppm) }\end{array}$ & Glycoform \\
\hline \multirow{33}{*}{$\mathrm{C} 1$} & \multirow{33}{*}{ IVLGNVTE } & 2 & 949.9274 & 949.9273 & -0.1 & HexNAc2Hex4 \\
\hline & & 2 & 1030.9538 & 1030.9521 & -1.6 & HexNAc2Hex 5 \\
\hline & & 2 & 1111.9802 & 1111.9794 & -0.7 & HexNAc2Hex6 \\
\hline & & 2 & 1193.0066 & 1193.0078 & 1.0 & HexNAc2Hex7 \\
\hline & & 2 & 1274.0330 & 1274.0343 & 1.0 & HexNAc2Hex 8 \\
\hline & & 2 & 970.4407 & 970.4389 & -1.9 & HexNAc3Hex3 \\
\hline & & 2 & 1051.4671 & 1051.4680 & 0.9 & HexNAc3Hex4 \\
\hline & & 2 & 1071.9804 & 1071.9826 & 2.1 & HexNAc4Hex3 \\
\hline & & 3 & 769.0069 & 769.0076 & 0.9 & HexNAc4Hex4 \\
\hline & & 3 & 823.0245 & 823.0255 & 1.2 & HexNAc4Hex 5 \\
\hline & & 3 & 782.6825 & 782.6848 & 2.9 & HexNAc5Hex3 \\
\hline & & 3 & 836.7001 & 836.7007 & 0.7 & HexNAc5Hex4 \\
\hline & & 3 & 890.7177 & 890.7178 & 0.1 & HexNAc5Hex 5 \\
\hline & & 2 & 962.4432 & 962.4426 & -0.6 & HexNAc3Hex2Fuc1 \\
\hline & & 2 & 1043.4696 & 1043.4679 & -1.6 & HexNAc3Hex3Fuc1 \\
\hline & & 2 & 1124.4960 & 1124.4959 & -0.1 & HexNAc3Hex4Fuc1 \\
\hline & & 3 & 804.0174 & 804.0183 & 1.1 & HexNAc3Hex5Fuc1 \\
\hline & & 3 & 858.0350 & 858.0325 & -2.9 & HexNAc3Hex6Fuc1 \\
\hline & & 3 & 763.6753 & 763.6752 & -0.1 & HexNAc4Hex3Fuc1 \\
\hline & & 3 & 817.6929 & 817.6906 & -2.8 & HexNAc4Hex4Fuc1 \\
\hline & & 3 & 871.7105 & 871.7112 & 0.8 & HexNAc4Hex5Fuc1 \\
\hline & & 3 & 831.3684 & 831.3681 & -0.4 & HexNAc5Hex3Fuc1 \\
\hline & & 3 & 885.3860 & 885.3867 & 0.8 & HexNAc5Hex4Fuc1 \\
\hline & & 3 & 939.4036 & 939.4048 & 1.3 & HexNAc5Hex5Fuc1 \\
\hline & & 3 & 993.4212 & 993.4223 & 1.1 & HexNAc5Hex6Fuc1 \\
\hline & & 3 & 899.0616 & 899.0610 & -0.7 & HexNAc6Hex3Fuc1 \\
\hline & & 3 & 953.0792 & 953.0810 & 1.9 & HexNAc6Hex4Fuc1 \\
\hline & & 3 & 1007.0968 & 1007.0967 & -0.1 & HexNAc6Hex5Fuc1 \\
\hline & & 3 & 1061.1144 & 1061.1159 & 1.4 & HexNAc6Hex6Fuc1 \\
\hline & & 3 & 966.7547 & 966.7535 & -1.2 & HexNAc7Hex3Fuc1 \\
\hline & & 3 & 1020.7723 & 1020.7737 & 1.4 & HexNAc7Hex4Fuc1 \\
\hline & & 3 & 1074.7899 & 1074.7882 & -1.6 & HexNAc7Hex5Fuc1 \\
\hline & & 3 & 1128.8075 & 1128.8090 & 1.3 & HexNAc7Hex6Fuc1 \\
\hline
\end{tabular}




\begin{tabular}{|c|c|c|c|c|c|c|}
\hline Domain & $\begin{array}{c}\text { Peptide } \\
\text { sequence }\end{array}$ & $\begin{array}{l}\text { Charge } \\
\text { state }\end{array}$ & $\begin{array}{c}\text { Theoretical } \\
\mathbf{m} / \mathbf{z}\end{array}$ & $\begin{array}{c}\text { Observed } \\
\mathbf{m} / \mathbf{z}\end{array}$ & $\begin{array}{c}\text { Mass } \\
\text { accuracy } \\
(\mathrm{ppm})\end{array}$ & Glycoform \\
\hline & & 3 & 1182.8251 & 1182.8237 & -1.2 & HexNAc7Hex7Fuc1 \\
\hline & & 3 & 920.3965 & 920.3943 & -2.4 & HexNAc4Hex5Fuc2 \\
\hline & & 3 & 934.0720 & 934.0716 & -0.4 & HexNAc5Hex4Fuc2 \\
\hline & & 3 & 988.0896 & 988.0898 & 0.2 & HexNAc5Hex5Fuc2 \\
\hline & & 3 & 920.0563 & 920.0538 & -2.7 & HexNAc4Hex5NeuAc1 \\
\hline & & 3 & 974.0739 & 974.0745 & 0.6 & HexNAc4Hex6NeuAc1 \\
\hline & & 3 & 1041.7671 & 1041.7664 & -0.7 & HexNAc5Hex6NeuAc1 \\
\hline & & 2 & 1270.0437 & 1270.0411 & -2.0 & HexNAc3Hex4Fuc1NeuAc1 \\
\hline & & 3 & 901.0492 & 901.0472 & -2.2 & HexNAc3Hex5Fuc1NeuAc1 \\
\hline & & 3 & 914.7247 & 914.7241 & -0.7 & HexNAc4Hex4Fuc1NeuAc1 \\
\hline & & 3 & 968.7423 & 968.7422 & -0.1 & HexNAc4Hex5Fuc1NeuAc1 \\
\hline & & 3 & 928.4002 & 928.4017 & 1.6 & HexNAc5Hex3Fuc1NeuAc1 \\
\hline & & 3 & 982.4178 & 982.4176 & -0.2 & HexNAc5Hex4Fuc1NeuAc1 \\
\hline & & 3 & 1036.4354 & 1036.4355 & 0.1 & HexNAc5Hex5Fuc1NeuAc1 \\
\hline & & 3 & 1090.4530 & 1090.4531 & 0.1 & HexNAc5Hex6Fuc1NeuAc1 \\
\hline & & 3 & 996.0934 & 996.0938 & 0.4 & HexNAc6Hex3Fuc1NeuAc1 \\
\hline & & 3 & 1225.8393 & 1225.8401 & 0.7 & HexNAc7Hex6Fuc1NeuAc1 \\
\hline & & 3 & 1279.8569 & 1279.8560 & -0.7 & HexNAc7Hex7Fuc1NeuAc1 \\
\hline & & 3 & 1017.4283 & 1017.4289 & 0.6 & HexNAc4Hex5Fuc2NeuAc1 \\
\hline & & 3 & 1031.1038 & 1031.1038 & 0.0 & HexNAc5Hex4Fuc2NeuAc1 \\
\hline & & 3 & 1065.7741 & 1065.7739 & -0.2 & HexNAc4Hex5Fuc1NeuAc2 \\
\hline & & 3 & 1079.4496 & 1079.4489 & -0.6 & HexNAc5Hex4Fuc1NeuAc2 \\
\hline & & 3 & 1133.4672 & 1133.4717 & 4.0 & HexNAc5Hex5Fuc1NeuAc2 \\
\hline & & 3 & 1187.4848 & 1187.4845 & -0.3 & HexNAc5Hex6Fuc1NeuAc2 \\
\hline & & 3 & 1201.1604 & 1201.1599 & -0.4 & HexNAc6Hex5Fuc1NeuAc2 \\
\hline & & 3 & 1309.1956 & 1309.1965 & 0.7 & HexNAc6Hex7Fuc1NeuAc2 \\
\hline & \multirow{13}{*}{$\begin{array}{l}\text { LTPLCVTLN } \\
\text { CTE }\end{array}$} & 2 & 994.9500 & 994.9497 & -0.3 & HexNAc2Hex1 \\
\hline & & 2 & 1075.9764 & 1075.9767 & 0.3 & HexNAc2Hex2 \\
\hline & & 2 & 1157.0028 & 1157.0028 & 0.0 & HexNAc2Hex3 \\
\hline & & 2 & 1238.0292 & 1238.0282 & -0.8 & HexNAc2Hex 4 \\
\hline & & 2 & 1319.0556 & 1319.0548 & -0.6 & HexNAc2Hex 5 \\
\hline & & 2 & 1400.0820 & 1400.0815 & -0.4 & HexNAc2Hex6 \\
\hline & & 2 & 1481.1084 & 1481.1072 & -0.8 & HexNAc2Hex7 \\
\hline & & 2 & 1562.1348 & 1562.1335 & -0.8 & HexNAc2Hex8 \\
\hline & & 2 & 1643.1612 & 1643.1660 & 2.9 & HexNAc2Hex9 \\
\hline & & 3 & 893.3817 & 893.3804 & -1.5 & HexNAc3Hex4 \\
\hline & & 3 & 947.3993 & 947.3978 & -1.6 & HexNAc3Hex 5 \\
\hline & & 3 & 1001.4169 & 1001.4161 & -0.8 & HexNAc3Hex6 \\
\hline & & 3 & 907.0572 & 907.0600 & 3.1 & HexNAc4Hex3 \\
\hline
\end{tabular}




\begin{tabular}{|c|c|c|c|c|c|c|}
\hline Domain & $\begin{array}{l}\text { Peptide } \\
\text { sequence }\end{array}$ & $\begin{array}{l}\text { Charge } \\
\text { state }\end{array}$ & $\begin{array}{c}\text { Theoretical } \\
\mathbf{m} / \mathbf{z}\end{array}$ & $\begin{array}{c}\text { Observed } \\
\mathbf{m} / \mathbf{z}\end{array}$ & $\begin{array}{c}\text { Mass } \\
\text { accuracy } \\
\text { (ppm) }\end{array}$ & Glycoform \\
\hline & & 3 & 961.0748 & 961.0727 & -2.2 & HexNAc4Hex4 \\
\hline & & 3 & 1015.0924 & 1015.0908 & -1.6 & HexNAc4Hex5 \\
\hline & & 3 & 1069.1100 & 1069.1112 & 1.1 & HexNAc4Hex6 \\
\hline & & 3 & 1123.1276 & 1123.1237 & -3.5 & HexNAc4Hex7 \\
\hline & & 3 & 1028.7679 & 1028.7697 & 1.7 & HexNAc5Hex4 \\
\hline & & 3 & 1082.7855 & 1082.7893 & 3.5 & HexNAc5Hex 5 \\
\hline & & 3 & 1136.8031 & 1136.8019 & -1.1 & HexNAc5Hex6 \\
\hline & & 3 & 1150.4787 & 1150.4750 & -3.2 & HexNAc6Hex5 \\
\hline & & 3 & 1258.5139 & 1258.5140 & 0.1 & HexNAc6Hex7 \\
\hline & & 2 & 1230.0317 & 1230.0341 & 2.0 & HexNAc2Hex3Fuc1 \\
\hline & & 2 & 1250.5450 & 1250.5385 & -5.2 & HexNAc3Hex2Fuc1 \\
\hline & & 2 & 1331.5714 & 1331.5698 & -1.2 & HexNAc3Hex3Fuc1 \\
\hline & & 3 & 942.0676 & 942.0692 & 1.7 & HexNAc3Hex4Fuc1 \\
\hline & & 3 & 996.0852 & 996.0840 & -1.2 & HexNAc3Hex5Fuc1 \\
\hline & & 3 & 1050.1028 & 1050.0997 & -3.0 & HexNAc3Hex6Fuc1 \\
\hline & & 3 & 955.7432 & 955.7424 & -0.8 & HexNAc4Hex3Fuc1 \\
\hline & & 3 & 1009.7608 & 1009.7551 & -5.6 & HexNAc4Hex4Fuc1 \\
\hline & & 3 & 1063.7784 & 1063.7786 & 0.2 & HexNAc4Hex5Fuc1 \\
\hline & & 3 & 1117.7960 & 1117.7996 & 3.2 & HexNAc4Hex6Fuc1 \\
\hline & & 3 & 1023.4363 & 1023.4362 & -0.1 & HexNAc5Hex3Fuc1 \\
\hline & & 3 & 1077.4539 & 1077.4536 & -0.3 & HexNAc5Hex4Fuc1 \\
\hline & & 3 & 1131.4715 & 1131.4709 & -0.5 & HexNAc5Hex5Fuc1 \\
\hline & & 3 & 1185.4891 & 1185.4880 & -0.9 & HexNAc5Hex6Fuc1 \\
\hline & & 3 & 1091.1294 & 1091.1284 & -0.9 & HexNAc6Hex3Fuc1 \\
\hline & & 3 & 1145.1470 & 1145.1467 & -0.3 & HexNAc6Hex4Fuc1 \\
\hline & & 3 & 1199.1646 & 1199.1626 & -1.7 & HexNAc6Hex5Fuc1 \\
\hline & & 3 & 1253.1822 & 1253.1799 & -1.8 & HexNAc6Hex6Fuc1 \\
\hline & & 3 & 1307.1998 & 1307.1993 & -0.4 & HexNAc6Hex7Fuc1 \\
\hline & & 3 & 1320.8754 & 1320.8708 & -3.5 & HexNAc7Hex6Fuc1 \\
\hline & & 3 & 1374.8930 & 1374.8929 & -0.1 & HexNAc7Hex7Fuc1 \\
\hline & & 3 & 990.4135 & 990.4139 & 0.4 & HexNAc3Hex4NeuAc1 \\
\hline & & 3 & 1044.4311 & 1044.4305 & -0.6 & HexNAc3Hex5NeuAc1 \\
\hline & & 3 & 1098.4487 & 1098.4482 & -0.5 & HexNAc3Hex6NeuAc1 \\
\hline & & 3 & 1058.1066 & 1058.1060 & -0.6 & HexNAc4Hex4NeuAc1 \\
\hline & & 3 & 1112.1242 & 1112.1213 & -2.6 & HexNAc4Hex5NeuAc1 \\
\hline & & 3 & 1166.1418 & 1166.1436 & 1.5 & HexNAc4Hex6NeuAc1 \\
\hline & & 3 & 1233.8349 & 1233.8376 & 2.2 & HexNAc5Hex6NeuAc1 \\
\hline & & 3 & 1039.0994 & 1039.0986 & -0.8 & HexNAc3Hex4Fuc1NeuAc1 \\
\hline & & 3 & 1093.1170 & 1093.1158 & -1.1 & HexNAc3Hex5Fuc1NeuAc1 \\
\hline & & 3 & 1147.1346 & 1147.1365 & 1.7 & HexNAc3Hex6Fuc1NeuAc1 \\
\hline & & 3 & 1106.7926 & 1106.7845 & -7.3 & HexNAc4Hex4Fuc1NeuAc1 \\
\hline & & 3 & 1160.8102 & 1160.8096 & -0.5 & HexNAc4Hex5Fuc1NeuAc1 \\
\hline & & 3 & 1174.4857 & 1174.4811 & -3.9 & HexNAc5Hex4Fuc1NeuAc1 \\
\hline
\end{tabular}




\begin{tabular}{|c|c|c|c|c|c|c|}
\hline Domain & $\begin{array}{l}\text { Peptide } \\
\text { sequence }\end{array}$ & $\begin{array}{c}\text { Charge } \\
\text { state }\end{array}$ & $\begin{array}{c}\text { Theoretical } \\
\mathbf{m} / \mathbf{z}\end{array}$ & $\begin{array}{c}\text { Observed } \\
\mathbf{m} / \mathbf{z}\end{array}$ & $\begin{array}{c}\text { Mass } \\
\text { accuracy } \\
(\mathrm{ppm})\end{array}$ & Glycoform \\
\hline & & 3 & 1228.5033 & 1228.5056 & 1.9 & HexNAc5Hex5Fuc1NeuAc1 \\
\hline & & 3 & 1282.5209 & 1282.5195 & -1.1 & HexNAc5Hex6Fuc1NeuAc1 \\
\hline & & 3 & 1242.1788 & 1242.1744 & -3.5 & HexNAc6Hex4Fuc1NeuAc1 \\
\hline & & 3 & 1296.1964 & 1296.1985 & 1.6 & HexNAc6Hex5Fuc1NeuAc1 \\
\hline & & 3 & 1350.2140 & 1350.2173 & 2.4 & HexNAc6Hex6Fuc1NeuAc1 \\
\hline & & 3 & 1404.2316 & 1404.2295 & -1.5 & HexNAc6Hex7Fuc1NeuAc1 \\
\hline & & 3 & 1471.9248 & 1471.9249 & 0.1 & HexNAc7Hex7Fuc1NeuAc1 \\
\hline & & 3 & 1257.8420 & 1257.8405 & -1.2 & HexNAc4Hex5Fuc1NeuAc2 \\
\hline & & 3 & 1379.5527 & 1379.5607 & 5.8 & HexNAc5Hex6Fuc1NeuAc2 \\
\hline & & 3 & 1501.2634 & 1501.2611 & -1.5 & HexNAc6Hex7Fuc1NeuAc2 \\
\hline & & 3 & 1598.2952 & 1598.2999 & 2.9 & HexNAc6Hex7Fuc1NeuAc3 \\
\hline \multirow{32}{*}{$\mathrm{V} 1 / \mathrm{V} 2$} & \multirow{2}{*}{ VNVTR } & 2 & 1064.9410 & 1064.9357 & -5.0 & HexNAc2Hex7 \\
\hline & & 2 & 1145.9674 & 1145.9569 & -9.2 & HexNAc2Hex8 \\
\hline & \multirow{30}{*}{ NCSFNITTE } & 2 & 1070.4170 & 1070.4184 & 1.3 & HexNAc2Hex4 \\
\hline & & 2 & 1151.4434 & 1151.4424 & -0.9 & HexNAc2Hex5 \\
\hline & & 2 & 1232.4698 & 1232.4681 & -1.4 & HexNAc2Hex6 \\
\hline & & 2 & 1313.4962 & 1313.4950 & -0.9 & HexNAc2Hex7 \\
\hline & & 2 & 1394.5226 & 1394.5210 & -1.1 & HexNAc2Hex 8 \\
\hline & & 2 & 1475.5490 & 1475.5510 & 1.4 & HexNAc2Hex9 \\
\hline & & 2 & 1334.0095 & 1334.0077 & -1.3 & HexNAc3Hex6 \\
\hline & & 3 & 1119.4214 & 1119.4191 & -2.1 & HexNAc4Hex9 \\
\hline & & 3 & 1173.4390 & 1173.4371 & -1.6 & HexNAc4Hex10 \\
\hline & & 3 & 1227.4566 & 1227.4543 & -1.9 & HexNAc4Hex11 \\
\hline & & 3 & 1281.4742 & 1281.4725 & -1.3 & HexNAc4Hex12 \\
\hline & & 3 & 1335.4918 & 1335.4890 & -2.1 & HexNAc4Hex13 \\
\hline & & 3 & 1389.5094 & 1389.5098 & 0.3 & HexNAc4Hex14 \\
\hline & & 3 & 1443.5270 & 1443.5238 & -2.2 & HexNAc4Hex15 \\
\hline & & 3 & 1497.5446 & 1497.5438 & -0.5 & HexNAc4Hex16 \\
\hline & & 3 & 1551.5622 & 1551.5675 & 3.4 & HexNAc4Hex17 \\
\hline & & 3 & 863.0089 & 863.0059 & -3.5 & HexNAc5Hex3 \\
\hline & & 2 & 1163.9593 & 1163.9580 & -1.1 & HexNAc3Hex3Fuc1 \\
\hline & & 2 & 1326.0121 & 1326.0090 & -2.3 & HexNAc3Hex5Fuc1 \\
\hline & & 2 & 1265.4990 & 1265.4984 & -0.5 & HexNAc4Hex3Fuc1 \\
\hline & & 2 & 1346.5254 & 1346.5244 & -0.7 & HexNAc4Hex4Fuc1 \\
\hline & & 2 & 1427.5518 & 1427.5544 & 1.8 & HexNAc4Hex5Fuc1 \\
\hline & & 3 & 911.6949 & 911.6932 & -1.9 & HexNAc5Hex3Fuc1 \\
\hline & & 3 & 965.7125 & 965.7109 & -1.7 & HexNAc5Hex4Fuc1 \\
\hline & & 3 & 1019.7301 & 1019.7287 & -1.4 & HexNAc5Hex5Fuc1 \\
\hline & & 3 & 1073.7477 & 1073.7478 & 0.1 & HexNAc5Hex6Fuc1 \\
\hline & & 3 & 979.3880 & 979.3873 & -0.7 & HexNAc6Hex3Fuc1 \\
\hline & & 3 & 1087.4232 & 1087.4236 & 0.4 & HexNAc6Hex5Fuc1 \\
\hline & & 3 & 1141.4408 & 1141.4385 & -2.0 & HexNAc6Hex6Fuc1 \\
\hline & & 3 & 1195.4584 & 1195.4568 & -1.3 & HexNAc6Hex7Fuc1 \\
\hline
\end{tabular}




\begin{tabular}{|c|c|c|c|c|c|c|}
\hline Domain & $\begin{array}{c}\text { Peptide } \\
\text { sequence }\end{array}$ & $\begin{array}{c}\text { Charge } \\
\text { state }\end{array}$ & $\begin{array}{c}\text { Theoretical } \\
\mathbf{m} / \mathbf{z}\end{array}$ & $\begin{array}{c}\text { Observed } \\
\mathbf{m} / \mathbf{z}\end{array}$ & $\begin{array}{c}\text { Mass } \\
\text { accuracy } \\
(\mathrm{ppm})\end{array}$ & Glycoform \\
\hline & & 3 & 1101.0987 & 1101.0971 & -1.5 & HexNAc7Hex4Fuc1 \\
\hline & & 3 & 1263.1515 & 1263.1489 & -2.1 & HexNAc7Hex7Fuc1 \\
\hline & & 2 & 1479.5572 & 1479.5564 & -0.5 & HexNAc3Hex6NeuAc1 \\
\hline & & 3 & 1000.3828 & 1000.3822 & -0.6 & HexNAc4Hex5NeuAc1 \\
\hline & & 2 & 1471.5598 & 1471.5656 & 3.9 & HexNAc3Hex5Fuc1NeuAc1 \\
\hline & & 3 & 1049.0687 & 1049.0680 & -0.7 & HexNAc4Hex5Fuc1NeuAc1 \\
\hline & & 3 & 1062.7443 & 1062.7449 & 0.6 & HexNAc5Hex4Fuc1NeuAc1 \\
\hline & & 3 & 1116.7619 & 1116.7622 & 0.3 & HexNAc5Hex5Fuc1NeuAc1 \\
\hline & & 3 & 1170.7795 & 1170.7789 & -0.5 & HexNAc5Hex6Fuc1NeuAc1 \\
\hline & & 3 & 1238.4726 & 1238.4725 & -0.1 & HexNAc6Hex6Fuc1NeuAc1 \\
\hline & & 3 & 1292.4902 & 1292.4865 & -2.9 & HexNAc6Hex7Fuc1NeuAc1 \\
\hline & & 3 & 1360.1833 & 1360.1816 & -1.2 & HexNAc7Hex7Fuc1NeuAc1 \\
\hline & & 3 & 1146.1005 & 1146.0986 & -1.7 & HexNAc4Hex5Fuc1NeuAc2 \\
\hline & & 3 & 1389.5220 & 1389.5194 & -1.9 & HexNAc6Hex7Fuc1NeuAc2 \\
\hline & \multirow{29}{*}{ LDIVSLNE(T) } & 2 & 897.9037 & 897.9006 & -3.5 & HexNAc2Hex3 \\
\hline & & 2 & 978.9301 & 978.9291 & -1.0 & HexNAc2Hex 4 \\
\hline & & 2 & 1059.9565 & 1059.9556 & -0.8 & HexNAc2Hex5 \\
\hline & & 2 & 1140.9829 & 1140.9813 & -1.4 & HexNAc2Hex6 \\
\hline & & 2 & 1222.0093 & 1222.0078 & -1.2 & HexNAc2Hex 7 \\
\hline & & 3 & 869.0262 & 869.0273 & 1.3 & HexNAc2Hex 8 \\
\hline & & 2 & 999.4434 & 999.4436 & 0.2 & HexNAc3Hex3 \\
\hline & & 2 & 1080.4698 & 1080.4698 & 0.0 & HexNAc3Hex4 \\
\hline & & 2 & 1161.4962 & 1161.4956 & -0.5 & HexNAc3Hex5 \\
\hline & & 2 & 1242.5226 & 1242.5226 & 0.0 & HexNAc3Hex6 \\
\hline & & 3 & 802.0176 & 802.0174 & -0.2 & HexNAc5Hex3 \\
\hline & & 3 & 856.0352 & 856.0317 & -4.1 & HexNAc5Hex4 \\
\hline & & 3 & 910.0528 & 910.0493 & -3.8 & HexNAc5Hex5 \\
\hline & & 3 & 964.0704 & 964.0695 & -0.9 & HexNAc5Hex6 \\
\hline & & 3 & 869.7108 & 869.7098 & -1.1 & HexNAc6Hex3 \\
\hline & & 3 & 923.7283 & 923.7269 & -1.5 & HexNAc6Hex4 \\
\hline & & 3 & 1085.7811 & 1085.7805 & -0.6 & HexNAc6Hex7 \\
\hline & & 2 & 1234.5251 & 1234.5240 & -0.9 & HexNAc3Hex5Fuc1 \\
\hline & & 2 & 1315.5515 & 1315.5586 & 5.4 & HexNAc3Hex6Fuc1 \\
\hline & & 2 & 1174.0120 & 1174.0079 & -3.5 & HexNAc4Hex3Fuc1 \\
\hline & & 3 & 837.0280 & 837.0248 & -3.8 & HexNAc4Hex4Fuc1 \\
\hline & & 3 & 891.0456 & 891.0466 & 1.1 & HexNAc4Hex5Fuc1 \\
\hline & & 3 & 850.7036 & 850.7024 & -1.4 & HexNAc5Hex3Fuc1 \\
\hline & & 3 & 904.7212 & 904.7178 & -3.8 & HexNAc5Hex4Fuc1 \\
\hline & & 3 & 958.7388 & 958.7407 & 2.0 & HexNAc5Hex5Fuc1 \\
\hline & & 3 & 1012.7564 & 1012.7563 & -0.1 & HexNAc5Hex6Fuc1 \\
\hline & & 3 & 918.3967 & 918.3962 & -0.5 & HexNAc6Hex3Fuc1 \\
\hline & & 3 & 972.4143 & 972.4145 & 0.2 & HexNAc6Hex4Fuc1 \\
\hline & & 3 & 1026.4319 & 1026.4320 & 0.1 & HexNAc6Hex5Fuc1 \\
\hline
\end{tabular}




\begin{tabular}{|c|c|c|c|c|c|c|}
\hline Domain & $\begin{array}{c}\text { Peptide } \\
\text { sequence }\end{array}$ & $\begin{array}{l}\text { Charge } \\
\text { state }\end{array}$ & $\begin{array}{c}\text { Theoretical } \\
\mathbf{m} / \mathbf{z}\end{array}$ & $\begin{array}{c}\text { Observed } \\
\mathbf{m} / \mathbf{z}\end{array}$ & $\begin{array}{c}\text { Mass } \\
\text { accuracy } \\
(\text { ppm) }\end{array}$ & Glycoform \\
\hline & & 3 & 1080.4495 & 1080.4493 & -0.2 & HexNAc6Hex6Fuc1 \\
\hline & & 3 & 1134.4671 & 1134.4655 & -1.4 & HexNAc6Hex7Fuc1 \\
\hline & & 3 & 1040.1074 & 1040.1062 & -1.2 & HexNAc7Hex4Fuc1 \\
\hline & & 3 & 1148.1426 & 1148.1434 & 0.7 & HexNAc7Hex6Fuc1 \\
\hline & & 3 & 1202.1602 & 1202.1589 & -1.1 & HexNAc7Hex7Fuc1 \\
\hline & & 2 & 1307.0439 & 1307.0438 & -0.1 & HexNAc3Hex5NeuAc1 \\
\hline & & 3 & 925.7159 & 925.7135 & -2.6 & HexNAc3Hex6NeuAc1 \\
\hline & & 2 & 1327.5572 & 1327.5653 & 6.1 & HexNAc4Hex4NeuAc1 \\
\hline & & 3 & 939.3914 & 939.3904 & -1.1 & HexNAc4Hex5NeuAc1 \\
\hline & & 3 & 1061.1022 & 1061.0968 & -5.1 & HexNAc5Hex6NeuAc1 \\
\hline & & 3 & 1182.8129 & 1182.8158 & 2.5 & HexNAc6Hex7NeuAc1 \\
\hline & & 3 & 988.0774 & 988.0766 & -0.8 & HexNAc4Hex5Fuc1NeuAc1 \\
\hline & & 3 & 1001.7530 & 1001.7591 & 6.1 & HexNAc5Hex4Fuc1NeuAc1 \\
\hline & & 3 & 1055.7706 & 1055.7687 & -1.8 & HexNAc5Hex5Fuc1NeuAc1 \\
\hline & & 3 & 1109.7882 & 1109.7848 & -3.1 & HexNAc5Hex6Fuc1NeuAc1 \\
\hline & & 3 & 1069.4461 & 1069.4482 & 2.0 & HexNAc6Hex4Fuc1NeuAc1 \\
\hline & & 3 & 1123.4637 & 1123.4631 & -0.5 & HexNAc6Hex5Fuc1NeuAc1 \\
\hline & & 3 & 1177.4813 & 1177.4810 & -0.3 & HexNAc6Hex6Fuc1NeuAc1 \\
\hline & & 3 & 1231.4989 & 1231.4995 & 0.5 & HexNAc6Hex7Fuc1NeuAc1 \\
\hline & & 3 & 1137.1392 & 1137.1381 & -1.0 & HexNAc7Hex4Fuc1NeuAc1 \\
\hline & & 3 & 1245.1744 & 1245.1680 & -5.1 & HexNAc7Hex6Fuc1NeuAc1 \\
\hline & & 3 & 1299.1920 & 1299.1925 & 0.4 & HexNAc7Hex7Fuc1NeuAc1 \\
\hline & & 3 & 1104.1164 & 1104.1194 & 2.7 & HexNAc5Hex5NeuAc2 \\
\hline & & 3 & 1158.1340 & 1158.1337 & -0.3 & HexNAc5Hex6NeuAc2 \\
\hline & & 3 & 1220.4955 & 1220.4987 & 2.6 & HexNAc6Hex5Fuc1NeuAc2 \\
\hline & & 3 & 1279.8447 & 1279.8461 & 1.1 & HexNAc6Hex7NeuAc2 \\
\hline & & 3 & 1206.8200 & 1206.8188 & -1.0 & HexNAc5Hex6Fuc1NeuAc2 \\
\hline & & 3 & 1274.5131 & 1274.5187 & 4.4 & HexNAc6Hex6Fuc1NeuAc2 \\
\hline & & 3 & 1328.5307 & 1328.5295 & -0.9 & HexNAc6Hex7Fuc1NeuAc2 \\
\hline & & 3 & 1396.2238 & 1396.2198 & -2.9 & HexNAc7Hex7Fuc1NeuAc2 \\
\hline & & 3 & 1425.5625 & 1425.5619 & -0.4 & HexNAc6Hex7Fuc1NeuAc3 \\
\hline & $\begin{array}{l}\text { LDIVSLNETD } \\
\text { DSETGNSSK }\end{array}$ & 4 & 1114.9494 & 1114.9473 & -1.9 & HexNAc4Hex 10 \\
\hline \multirow{10}{*}{$\mathrm{C} 2$} & \multirow{10}{*}{$\begin{array}{c}\text { LINCNTSALT } \\
\text { QACPK }\end{array}$} & 3 & 861.7189 & 861.7194 & 0.6 & HexNAc2Hex3 \\
\hline & & 3 & 915.7365 & 915.7371 & 0.7 & HexNAc2Hex4 \\
\hline & & 3 & 969.7541 & 969.7545 & 0.4 & HexNAc2Hex 5 \\
\hline & & 3 & 1023.7717 & 1023.7709 & -0.8 & HexNAc2Hex6 \\
\hline & & 3 & 1077.7893 & 1077.7896 & 0.3 & HexNAc2Hex7 \\
\hline & & 3 & 1131.8069 & 1131.8053 & -1.4 & HexNAc2Hex8 \\
\hline & & 3 & 1185.8245 & 1185.8197 & -4.0 & HexNAc2Hex9 \\
\hline & & 3 & 1239.8421 & 1239.8445 & 1.9 & HexNAc2Hex10 \\
\hline & & 3 & 929.4121 & 929.4117 & -0.4 & HexNAc3Hex3 \\
\hline & & 3 & 983.4297 & 983.4266 & -3.2 & HexNAc3Hex 4 \\
\hline
\end{tabular}




\begin{tabular}{|c|c|c|c|c|c|c|}
\hline Domain & $\begin{array}{l}\text { Peptide } \\
\text { sequence }\end{array}$ & $\begin{array}{l}\text { Charge } \\
\text { state }\end{array}$ & $\begin{array}{c}\text { Theoretical } \\
\mathbf{m} / \mathbf{z}\end{array}$ & $\begin{array}{c}\text { Observed } \\
\mathbf{m} / \mathbf{z}\end{array}$ & $\begin{array}{c}\text { Mass } \\
\text { accuracy } \\
\text { (ppm) }\end{array}$ & Glycoform \\
\hline & & 3 & 1037.4473 & 1037.4463 & -1.0 & HexNAc3Hex 5 \\
\hline & & 3 & 1091.4649 & 1091.4644 & -0.5 & HexNAc3Hex6 \\
\hline & & 3 & 997.1052 & 997.1053 & 0.1 & HexNAc4Hex3 \\
\hline & & 3 & 1051.1228 & 1051.1278 & 4.8 & HexNAc4Hex4 \\
\hline & & 3 & 1105.1404 & 1105.1404 & 0.0 & HexNAc4Hex5 \\
\hline & & 3 & 1159.1580 & 1159.1528 & -4.5 & HexNAc4Hex6 \\
\hline & & 3 & 1064.7983 & 1064.7966 & -1.6 & HexNAc5Hex3 \\
\hline & & 3 & 1172.8335 & 1172.8317 & -1.5 & HexNAc5Hex5 \\
\hline & & 3 & 1348.5619 & 1348.5615 & -0.3 & HexNAc6Hex7 \\
\hline & & 3 & 910.4049 & 910.4052 & 0.3 & HexNAc2Hex3Fuc1 \\
\hline & & 3 & 964.4225 & 964.4230 & 0.5 & HexNAc2Hex4Fuc1 \\
\hline & & 3 & 978.0980 & 978.0933 & -4.8 & HexNAc3Hex3Fuc1 \\
\hline & & 3 & 1032.1156 & 1032.1125 & -3.0 & HexNAc3Hex4Fuc1 \\
\hline & & 3 & 1086.1332 & 1086.1328 & -0.4 & HexNAc3Hex5Fuc1 \\
\hline & & 3 & 1140.1508 & 1140.1499 & -0.8 & HexNAc3Hex6Fuc1 \\
\hline & & 3 & 1045.7912 & 1045.7904 & -0.8 & HexNAc4Hex3Fuc1 \\
\hline & & 3 & 1099.8088 & 1099.8080 & -0.7 & HexNAc4Hex4Fuc1 \\
\hline & & 3 & 1153.8264 & 1153.8269 & 0.4 & HexNAc4Hex5Fuc1 \\
\hline & & 3 & 1207.8440 & 1207.8446 & 0.5 & HexNAc4Hex6Fuc1 \\
\hline & & 3 & 1113.4843 & 1113.4835 & -0.7 & HexNAc5Hex3Fuc1 \\
\hline & & 3 & 1167.5019 & 1167.5004 & -1.3 & HexNAc5Hex4Fuc1 \\
\hline & & 3 & 1221.5195 & 1221.5197 & 0.2 & HexNAc5Hex5Fuc1 \\
\hline & & 3 & 1275.5371 & 1275.5370 & -0.1 & HexNAc5Hex6Fuc1 \\
\hline & & 3 & 1181.1774 & 1181.1776 & 0.2 & HexNAc6Hex3Fuc1 \\
\hline & & 3 & 1235.1950 & 1235.1921 & -2.3 & HexNAc6Hex4Fuc1 \\
\hline & & 3 & 1289.2126 & 1289.2107 & -1.5 & HexNAc6Hex5Fuc1 \\
\hline & & 3 & 1343.2302 & 1343.2277 & -1.9 & HexNAc6Hex6Fuc1 \\
\hline & & 3 & 1397.2478 & 1397.2455 & -1.6 & HexNAc6Hex7Fuc1 \\
\hline & & 3 & 1248.8706 & 1248.8693 & -1.0 & HexNAc7Hex3Fuc1 \\
\hline & & 3 & 1356.9058 & 1356.9030 & -2.1 & HexNAc7Hex5Fuc1 \\
\hline & & 3 & 1410.9234 & 1410.9200 & -2.4 & HexNAc7Hex6Fuc1 \\
\hline & & 3 & 1464.9410 & 1464.9403 & -0.5 & HexNAc7Hex7Fuc1 \\
\hline & & 3 & 1080.4615 & 1080.4601 & -1.3 & HexNAc3Hex4NeuAc1 \\
\hline & & 3 & 1134.4791 & 1134.4800 & 0.8 & HexNAc3Hex5NeuAc1 \\
\hline & & 3 & 1188.4967 & 1188.4955 & -1.0 & HexNAc3Hex6NeuAc1 \\
\hline & & 3 & 1202.1722 & 1202.1689 & -2.7 & HexNAc4Hex5NeuAc1 \\
\hline & & 3 & 1129.1474 & 1129.1438 & -3.2 & HexNAc3Hex4Fuc1NeuAc1 \\
\hline & & 3 & 1183.1650 & 1183.1637 & -1.1 & HexNAc3Hex5Fuc1NeuAc1 \\
\hline & & 3 & 1237.1826 & 1237.1781 & -3.6 & HexNAc3Hex6Fuc1NeuAc1 \\
\hline & & 3 & 1196.8406 & 1196.8369 & -3.1 & HexNAc4Hex4Fuc1NeuAc1 \\
\hline & & 3 & 1250.8582 & 1250.8564 & -1.4 & HexNAc4Hex5Fuc1NeuAc1 \\
\hline & & 3 & 1264.5337 & 1264.5349 & 0.9 & HexNAc5Hex4Fuc1NeuAc1 \\
\hline & & 3 & 1318.5513 & 1318.5467 & -3.5 & HexNAc5Hex5Fuc1NeuAc1 \\
\hline
\end{tabular}




\begin{tabular}{|c|c|c|c|c|c|c|}
\hline Domain & $\begin{array}{l}\text { Peptide } \\
\text { sequence }\end{array}$ & $\begin{array}{c}\text { Charge } \\
\text { state }\end{array}$ & $\begin{array}{l}\text { Theoretical } \\
\mathbf{m} / \mathbf{z}\end{array}$ & $\begin{array}{c}\text { Observed } \\
\mathbf{m} / \mathbf{z}\end{array}$ & $\begin{array}{c}\text { Mass } \\
\text { accuracy } \\
(\mathbf{p p m}) \\
\end{array}$ & Glycoform \\
\hline & & 3 & 1332.2268 & 1332.2189 & -5.9 & HexNAc6Hex4Fuc1NeuAc1 \\
\hline & & 3 & 1386.2444 & 1386.2433 & -0.8 & HexNAc6Hex5Fuc1NeuAc1 \\
\hline & & 3 & 1440.2620 & 1440.2576 & -3.1 & HexNAc6Hex6Fuc1NeuAc1 \\
\hline & & 3 & 1494.2796 & 1494.2759 & -2.5 & HexNAc6Hex7Fuc1NeuAc1 \\
\hline & & 3 & 1507.9552 & 1507.9597 & 3.0 & HexNAc7Hex6Fuc1NeuAc1 \\
\hline & & 3 & 1561.9728 & 1561.9735 & 0.4 & HexNAc7Hex7Fuc1NeuAc1 \\
\hline & \multirow{20}{*}{$\begin{array}{c}\text { TFNGTGPCH } \\
\text { NVSTVQCTH- } \\
\text { GIK }\end{array}$} & 4 & 1187.7332 & 1187.7319 & -1.1 & HexNAc4Hex10 \\
\hline & & 4 & 1228.2464 & 1228.2490 & 2.1 & HexNAc4Hex11 \\
\hline & & 4 & 1268.7596 & 1268.7571 & -2.0 & HexNAc4Hex12 \\
\hline & & 4 & 1309.2728 & 1309.2703 & -1.9 & HexNAc4Hex13 \\
\hline & & 4 & 1349.7860 & 1349.7811 & -3.6 & HexNAc4Hex14 \\
\hline & & 4 & 1390.2992 & 1390.2907 & -6.1 & HexNAc4Hex15 \\
\hline & & 4 & 1430.8124 & 1430.8114 & -0.7 & HexNAc4Hex16 \\
\hline & & 4 & 1238.5031 & 1238.5056 & 2.0 & HexNAc5Hex 10 \\
\hline & & 4 & 1319.5295 & 1319.5317 & 1.7 & HexNAc5Hex12 \\
\hline & & 4 & 1315.5308 & 1315.5358 & 3.8 & HexNAc5Hex11Fuc1 \\
\hline & & 4 & 1351.7901 & 1351.7896 & -0.4 & HexNAc5Hex11NeuAc1 \\
\hline & & 4 & 1392.3033 & 1392.2971 & -4.5 & HexNAc5Hex12NeuAc1 \\
\hline & & 4 & 1432.8165 & 1432.8099 & -4.6 & HexNAc5Hex13NeuAc1 \\
\hline & & 4 & 1473.3297 & 1473.3228 & -4.7 & HexNAc5Hex14NeuAc1 \\
\hline & & 4 & 1513.8429 & 1513.8474 & 3.0 & HexNAc5Hex 15NeuAc1 \\
\hline & & 4 & 1402.5600 & 1402.5562 & -2.7 & HexNAc6Hex11NeuAc1 \\
\hline & & 4 & 1483.5864 & 1483.5924 & 4.0 & HexNAc6Hex13NeuAc1 \\
\hline & & 4 & 1388.3046 & 1388.2929 & -8.4 & $\begin{array}{c}\text { HexNAc5Hex11Fuc1NeuAc } \\
1\end{array}$ \\
\hline & & 4 & 1509.8442 & 1509.8516 & 4.9 & $\begin{array}{c}\text { HexNAc5Hex14Fuc1NeuAc } \\
1\end{array}$ \\
\hline & & 4 & 1479.5877 & 1479.5806 & -4.8 & $\begin{array}{c}\text { HexNAc6Hex12Fuc1NeuAc } \\
1\end{array}$ \\
\hline & \multirow{15}{*}{ SENLTNNVK } & 2 & 611.3015 & 611.3011 & -0.7 & HexNAc1 \\
\hline & & 3 & 637.6160 & 637.6150 & -1.6 & HexNAc2Hex3 \\
\hline & & 3 & 691.6336 & 691.6329 & -1.0 & HexNAc2Hex4 \\
\hline & & 3 & 745.6512 & 745.6505 & -0.9 & HexNAc2Hex5 \\
\hline & & 3 & 799.6688 & 799.6676 & -1.5 & HexNAc2Hex6 \\
\hline & & 3 & 853.6864 & 853.6895 & 3.6 & HexNAc2Hex7 \\
\hline & & 3 & 907.7040 & 907.7062 & 2.4 & HexNAc2Hex 8 \\
\hline & & 2 & 1442.0788 & 1442.0764 & -1.7 & HexNAc2Hex9 \\
\hline & & 3 & 705.3092 & 705.3088 & -0.6 & HexNAc3Hex3 \\
\hline & & 3 & 759.3268 & 759.3260 & -1.1 & HexNAc3Hex4 \\
\hline & & 3 & 813.3444 & 813.3433 & -1.4 & HexNAc3Hex 5 \\
\hline & & 3 & 867.3620 & 867.3612 & -0.9 & HexNAc3Hex6 \\
\hline & & 3 & 773.0023 & 773.0018 & -0.6 & HexNAc4Hex3 \\
\hline & & 3 & 827.0199 & 827.0194 & -0.6 & HexNAc4Hex4 \\
\hline & & 3 & 881.0375 & 881.0366 & -1.0 & HexNAc4Hex5 \\
\hline
\end{tabular}




\begin{tabular}{|c|c|c|c|c|c|c|}
\hline Domain & $\begin{array}{l}\text { Peptide } \\
\text { sequence }\end{array}$ & $\begin{array}{c}\text { Charge } \\
\text { state }\end{array}$ & $\begin{array}{c}\text { Theoretical } \\
\mathbf{m} / \mathbf{z}\end{array}$ & $\begin{array}{c}\text { Observed } \\
\mathbf{m} / \mathbf{z}\end{array}$ & $\begin{array}{c}\text { Mass } \\
\text { accuracy } \\
(\mathrm{ppm})\end{array}$ & Glycoform \\
\hline & & 3 & 935.0551 & 935.0538 & -1.4 & HexNAc4Hex6 \\
\hline & & 3 & 840.6954 & 840.6946 & -1.0 & HexNAc5Hex3 \\
\hline & & 3 & 894.7130 & 894.7126 & -0.4 & HexNAc5Hex4 \\
\hline & & 3 & 948.7306 & 948.7283 & -2.4 & HexNAc5Hex 5 \\
\hline & & 3 & 862.0303 & 862.0305 & 0.2 & HexNAc3Hex5Fuc1 \\
\hline & & 3 & 821.6883 & 821.6857 & -3.2 & HexNAc4Hex3Fuc1 \\
\hline & & 2 & 1394.0816 & 1394.0833 & 1.2 & HexNAc4Hex5Fuc1 \\
\hline & & 3 & 889.3814 & 889.3771 & -4.8 & HexNAc5Hex3Fuc1 \\
\hline & & 3 & 910.3762 & 910.3757 & -0.5 & HexNAc3Hex5NeuAc1 \\
\hline & & 3 & 964.3938 & 964.3938 & 0.0 & HexNAc3Hex6NeuAc1 \\
\hline & & 3 & 1018.4114 & 1018.4105 & -0.9 & HexNAc3Hex7NeuAc1 \\
\hline & & 3 & 924.0517 & 924.0528 & 1.2 & HexNAc4Hex4NeuAc1 \\
\hline & & 3 & 978.0693 & 978.0686 & -0.7 & HexNAc4Hex5NeuAc1 \\
\hline & & 3 & 1032.0869 & 1032.0884 & 1.5 & HexNAc4Hex6NeuAc1 \\
\hline & & 3 & 1045.7624 & 1045.7646 & 2.1 & HexNAc5Hex5NeuAc1 \\
\hline & & 3 & 1099.7800 & 1099.7815 & 1.4 & HexNAc5Hex6NeuAc1 \\
\hline & & 3 & 1059.4380 & 1059.4366 & -1.3 & HexNAc6Hex4NeuAc1 \\
\hline & & 3 & 959.0621 & 959.0621 & 0.0 & HexNAc3Hex5Fuc1NeuAc1 \\
\hline & & 3 & 1013.0797 & 1013.0799 & 0.2 & HexNAc3Hex6Fuc1NeuAc1 \\
\hline & & 3 & 1026.7553 & 1026.7546 & -0.7 & HexNAc4Hex5Fuc1NeuAc1 \\
\hline & & 3 & 1080.7729 & 1080.7710 & -1.8 & HexNAc4Hex6Fuc1NeuAc1 \\
\hline & & 3 & 1040.4308 & 1040.4298 & -1.0 & HexNAc5Hex4Fuc1NeuAc1 \\
\hline & & 3 & 1148.4660 & 1148.4717 & 5.0 & HexNAc5Hex6Fuc1NeuAc1 \\
\hline & & 3 & 1075.1011 & 1075.0994 & -1.6 & HexNAc4Hex5NeuAc2 \\
\hline & & 3 & 1129.1187 & 1129.1167 & -1.8 & HexNAc4Hex6NeuAc2 \\
\hline & \multirow{9}{*}{ TIIVHLNR(S) } & 2 & 929.4568 & 929.4564 & -0.4 & HexNAc2Hex3 \\
\hline & & 2 & 1010.4832 & 1010.4823 & -0.9 & HexNAc2Hex4 \\
\hline & & 2 & 1091.5096 & 1091.5079 & -1.6 & HexNAc2Hex 5 \\
\hline & & 2 & 1172.5360 & 1172.5364 & 0.3 & HexNAc2Hex6 \\
\hline & & 2 & 1253.5624 & 1253.5620 & -0.3 & HexNAc2Hex7 \\
\hline & & 2 & 1334.5888 & 1334.5882 & -0.4 & HexNAc2Hex 8 \\
\hline & & 2 & 1415.6152 & 1415.6160 & 0.6 & HexNAc2Hex9 \\
\hline & & 2 & 1030.9965 & 1030.9993 & 2.7 & HexNAc3Hex3 \\
\hline & & 2 & 1112.0229 & 1112.0223 & -0.5 & HexNAc3Hex4 \\
\hline \multirow{9}{*}{ V3 } & \multirow{9}{*}{$\begin{array}{c}\text { SIEIVCVRPN } \\
\text { NNTR }\end{array}$} & 3 & 909.4147 & 909.4167 & 2.2 & HexNAc2Hex4 \\
\hline & & 3 & 963.4323 & 963.4360 & 3.8 & HexNAc2Hex5 \\
\hline & & 3 & 1017.4499 & 1017.4511 & 1.2 & HexNAc2Hex6 \\
\hline & & 3 & 1071.4675 & 1071.4640 & -3.3 & HexNAc2Hex7 \\
\hline & & 3 & 1125.4851 & 1125.4865 & 1.2 & HexNAc2Hex 8 \\
\hline & & 3 & 1179.5027 & 1179.4974 & -4.5 & HexNAc2Hex9 \\
\hline & & 3 & 923.0902 & 923.0918 & 1.7 & HexNAc3Hex3 \\
\hline & & 3 & 977.1078 & 977.1080 & 0.2 & HexNAc3Hex4 \\
\hline & & 3 & 1085.1430 & 1085.1450 & 1.8 & HexNAc3Hex6 \\
\hline
\end{tabular}




\begin{tabular}{|c|c|c|c|c|c|c|}
\hline Domain & $\begin{array}{l}\text { Peptide } \\
\text { sequence }\end{array}$ & $\begin{array}{l}\text { Charge } \\
\text { state }\end{array}$ & $\begin{array}{c}\text { Theoretical } \\
\mathbf{m} / \mathbf{z}\end{array}$ & $\begin{array}{c}\text { Observed } \\
\mathbf{m} / \mathbf{z}\end{array}$ & $\begin{array}{c}\text { Mass } \\
\text { accuracy } \\
\text { (ppm) }\end{array}$ & Glycoform \\
\hline & & 3 & 990.7833 & 990.7861 & 2.8 & HexNAc4Hex3 \\
\hline & & 3 & 1044.8009 & 1044.8061 & 5.0 & HexNAc4Hex4 \\
\hline & & 3 & 1098.8185 & 1098.8252 & 6.1 & HexNAc4Hex5 \\
\hline & & 3 & 1152.8361 & 1152.8405 & 3.8 & HexNAc4Hex6 \\
\hline & & 3 & 1058.4765 & 1058.4802 & 3.5 & HexNAc5Hex3 \\
\hline & & 3 & 1112.4941 & 1112.5009 & 6.1 & HexNAc5Hex 4 \\
\hline & & 3 & 1166.5117 & 1166.5141 & 2.1 & HexNAc5Hex5 \\
\hline & & 3 & 971.7762 & 971.7787 & 2.6 & HexNAc3Hex3Fuc1 \\
\hline & & 3 & 1025.7938 & 1025.7961 & 2.2 & HexNAc3Hex4Fuc1 \\
\hline & & 3 & 1079.8114 & 1079.8141 & 2.5 & HexNAc3Hex5Fuc1 \\
\hline & & 3 & 1133.8290 & 1133.8330 & 3.5 & HexNAc3Hex6Fuc1 \\
\hline & & 3 & 1039.4693 & 1039.4716 & 2.2 & HexNAc4Hex3Fuc1 \\
\hline & & 3 & 1093.4869 & 1093.4899 & 2.7 & HexNAc4Hex4Fuc1 \\
\hline & & 3 & 1147.5045 & 1147.5064 & 1.7 & HexNAc4Hex5Fuc1 \\
\hline & & 3 & 1201.5221 & 1201.5191 & -2.5 & HexNAc4Hex6Fuc1 \\
\hline & & 3 & 1107.1624 & 1107.1648 & 2.2 & HexNAc5Hex3Fuc1 \\
\hline & & 3 & 1161.1800 & 1161.1823 & 2.0 & HexNAc5Hex4Fuc1 \\
\hline & & 3 & 1215.1976 & 1215.2002 & 2.1 & HexNAc5Hex5Fuc1 \\
\hline & & 3 & 1269.2152 & 1269.2185 & 2.6 & HexNAc5Hex6Fuc1 \\
\hline & & 3 & 1174.8556 & 1174.8583 & 2.3 & HexNAc6Hex3Fuc1 \\
\hline & & 3 & 1228.8732 & 1228.8733 & 0.1 & HexNAc6Hex4Fuc1 \\
\hline & & 3 & 1282.8908 & 1282.8919 & 0.9 & HexNAc6Hex5Fuc1 \\
\hline & & 3 & 1336.9084 & 1336.9128 & 3.3 & HexNAc6Hex6Fuc1 \\
\hline & & 3 & 1242.5487 & 1242.5496 & 0.7 & HexNAc7Hex3Fuc1 \\
\hline & & 3 & 1296.5663 & 1296.5609 & -4.2 & HexNAc7Hex4Fuc1 \\
\hline & & 3 & 1350.5839 & 1350.5817 & -1.6 & HexNAc7Hex5Fuc1 \\
\hline & & 3 & 1404.6015 & 1404.6041 & 1.9 & HexNAc7Hex6Fuc1 \\
\hline & & 3 & 1196.1905 & 1196.1845 & -5.0 & HexNAc4Hex5Fuc2 \\
\hline & & 3 & 1209.8660 & 1209.8617 & -3.6 & HexNAc5Hex4Fuc2 \\
\hline & & 3 & 1263.8836 & 1263.8816 & -1.6 & HexNAc5Hex5Fuc2 \\
\hline & & 3 & 1317.9012 & 1317.9016 & 0.3 & HexNAc5Hex6Fuc2 \\
\hline & & 3 & 1223.5415 & 1223.5410 & -0.4 & HexNAc6Hex3Fuc2 \\
\hline & & 3 & 1258.5520 & 1258.5547 & 2.1 & HexNAc5Hex4Fuc3 \\
\hline & & 3 & 1128.1572 & 1128.1576 & 0.4 & HexNAc3Hex5NeuAc1 \\
\hline & & 3 & 1195.8503 & 1195.8517 & 1.2 & HexNAc4Hex5NeuAc1 \\
\hline & & 3 & 1176.8432 & 1176.8439 & 0.6 & HexNAc3Hex5Fuc1NeuAc1 \\
\hline & & 3 & 1230.8608 & 1230.8601 & -0.6 & HexNAc3Hex6Fuc1NeuAc1 \\
\hline & & 3 & 1190.5187 & 1190.5203 & 1.3 & HexNAc4Hex4Fuc1NeuAc1 \\
\hline & & 3 & 1244.5363 & 1244.5372 & 0.7 & HexNAc4Hex5Fuc1NeuAc1 \\
\hline & & 3 & 1204.1942 & 1204.1940 & -0.2 & HexNAc5Hex3Fuc1NeuAc1 \\
\hline & & 3 & 1258.2118 & 1258.2118 & 0.0 & HexNAc5Hex4Fuc1NeuAc1 \\
\hline & & 3 & 1312.2294 & 1312.2306 & 0.9 & HexNAc5Hex5Fuc1NeuAc1 \\
\hline & & 3 & 1366.2470 & 1366.2460 & -0.7 & HexNAc5Hex6Fuc1NeuAc1 \\
\hline
\end{tabular}




\begin{tabular}{|c|c|c|c|c|c|c|}
\hline Domain & $\begin{array}{l}\text { Peptide } \\
\text { sequence }\end{array}$ & $\begin{array}{l}\text { Charge } \\
\text { state }\end{array}$ & $\begin{array}{c}\text { Theoretical } \\
\mathbf{m} / \mathbf{z}\end{array}$ & $\begin{array}{c}\text { Observed } \\
\mathbf{m} / \mathbf{z}\end{array}$ & $\begin{array}{c}\text { Mass } \\
\text { accuracy } \\
\text { (ppm) }\end{array}$ & Glycoform \\
\hline & & 3 & 1271.8874 & 1271.8923 & 3.9 & HexNAc6Hex3Fuc1NeuAc1 \\
\hline & & 3 & 1379.9226 & 1379.9144 & -5.9 & HexNAc6Hex5Fuc1NeuAc1 \\
\hline & & 3 & 1433.9402 & 1433.9397 & -0.3 & HexNAc6Hex6Fuc1NeuAc1 \\
\hline & & 3 & 1293.2223 & 1293.2233 & 0.8 & HexNAc4Hex5Fuc2NeuAc1 \\
\hline & & 3 & 1306.8978 & 1306.8978 & 0.0 & HexNAc5Hex4Fuc2NeuAc1 \\
\hline & & 3 & 1341.5681 & 1341.5693 & 0.9 & HexNAc4Hex5Fuc1NeuAc2 \\
\hline & & 3 & 1355.2436 & 1355.2461 & 1.8 & HexNAc5Hex4Fuc1NeuAc2 \\
\hline & & 3 & 1409.2612 & 1409.2684 & 5.1 & HexNAc5Hex5Fuc1NeuAc2 \\
\hline & & 3 & 1463.2788 & 1463.2786 & $\begin{array}{c}-0.1 \\
\end{array}$ & HexNAc5Hex6Fuc1NeuAc2 \\
\hline \multirow{23}{*}{$\mathrm{C} 3$} & \multirow{8}{*}{ QAHCNISR } & 3 & 734.6335 & 734.6343 & 1.1 & HexNAc2Hex 5 \\
\hline & & 3 & 788.6511 & 788.6534 & 2.9 & HexNAc2Hex6 \\
\hline & & 3 & 842.6687 & 842.6730 & 5.1 & HexNAc2Hex7 \\
\hline & & 3 & 829.6777 & 829.6803 & 3.1 & HexNAc5Hex3 \\
\hline & & 3 & 883.6953 & 883.6965 & 1.4 & HexNAc5Hex4 \\
\hline & & 3 & 937.7129 & 937.7134 & 0.5 & HexNAc5Hex5 \\
\hline & & 3 & 932.3813 & 932.3878 & 7.0 & HexNAc5Hex4Fuc1 \\
\hline & & 3 & 986.3989 & 986.3989 & 0.0 & HexNAc5Hex5Fuc1 \\
\hline & \multirow{4}{*}{ TNWTKTLR - } & 3 & 746.0002 & 745.9973 & -3.9 & HexNAc2Hex5 \\
\hline & & 3 & 800.0178 & 800.0176 & -0.2 & HexNAc2Hex6 \\
\hline & & 3 & 854.0354 & 854.0351 & -0.4 & HexNAc2Hex7 \\
\hline & & 3 & 908.0530 & 908.0597 & 7.4 & HexNAc2Hex 8 \\
\hline & \multirow{11}{*}{ NITFK } & 2 & 838.8666 & 838.8664 & -0.2 & HexNAc2Hex4 \\
\hline & & 2 & 919.8930 & 919.8927 & -0.3 & HexNAc2Hex5 \\
\hline & & 2 & 1000.9194 & 1000.9189 & -0.5 & HexNAc2Hex6 \\
\hline & & 2 & 859.3799 & 859.3799 & 0.0 & HexNAc3Hex3 \\
\hline & & 2 & 940.4063 & 940.4051 & -1.3 & HexNAc3Hex4 \\
\hline & & 2 & 1021.4327 & 1021.4329 & 0.2 & HexNAc3Hex 5 \\
\hline & & 2 & 1122.9724 & 1122.9701 & -2.0 & HexNAc4Hex 5 \\
\hline & & 2 & 1062.4593 & 1062.4591 & -0.2 & HexNAc5Hex3 \\
\hline & & 2 & 1085.9540 & 1085.9535 & -0.5 & HexNAc3Hex4NeuAc1 \\
\hline & & 2 & 1248.0068 & 1248.0081 & 1.0 & HexNAc3Hex6NeuAc1 \\
\hline & & 2 & 1268.5201 & 1268.5201 & 0.0 & HexNAc4Hex5NeuAc1 \\
\hline \multirow{11}{*}{ V5 } & \multirow{11}{*}{$\begin{array}{c}\text { DGGSTNDST } \\
\text { NNNTEIFRPA } \\
\text { GGDMR }\end{array}$} & 3 & 1248.1761 & 1248.1846 & 6.8 & HexNAc2Hex 5 \\
\hline & & 3 & 1302.1937 & 1302.1947 & 0.8 & HexNAc2Hex6 \\
\hline & & 3 & 1356.2113 & 1356.2183 & 5.2 & HexNAc2Hex7 \\
\hline & & 3 & 1207.8340 & 1207.8414 & 6.1 & HexNAc3Hex3 \\
\hline & & 3 & 1324.2131 & 1324.2144 & 1.0 & HexNAc4Hex3Fuc1 \\
\hline & & 3 & 1378.2307 & 1378.2260 & -3.4 & HexNAc4Hex4Fuc1 \\
\hline & & 3 & 1391.9063 & 1391.9076 & 0.9 & HexNAc5Hex3Fuc1 \\
\hline & & 3 & 1445.9239 & 1445.9291 & 3.6 & HexNAc5Hex4Fuc1 \\
\hline & & 3 & 1499.9415 & 1499.9413 & -0.1 & HexNAc5Hex5Fuc1 \\
\hline & & 3 & 1475.2625 & 1475.2634 & 0.6 & HexNAc4Hex4Fuc1NeuAc1 \\
\hline & & 4 & 1147.2119 & 1147.2189 & 6.1 & HexNAc4Hex5Fuc1NeuAc1 \\
\hline
\end{tabular}




\begin{tabular}{|ccccccc|}
\hline Domain & $\begin{array}{c}\text { Peptide } \\
\text { sequence }\end{array}$ & $\begin{array}{c}\text { Charge } \\
\text { state }\end{array}$ & $\begin{array}{c}\text { Theoretical } \\
\mathbf{m} / \mathbf{z}\end{array}$ & $\begin{array}{c}\text { Observed } \\
\mathbf{m} / \mathbf{z}\end{array}$ & $\begin{array}{c}\text { Mass } \\
\text { accuracy } \\
\text { (ppm) }\end{array}$ & Glycoform \\
\hline & & 3 & 1542.9557 & 1542.9504 & -3.4 & HexNAc5Hex4Fuc1NeuAc1 \\
\cline { 2 - 7 } & & 3 & 1596.9733 & 1596.9758 & 1.6 & HexNAc5Hex5Fuc1NeuAc1 \\
\hline
\end{tabular}


Table S4. List of identified glycopeptides associated with 93TH975 gp120.

\begin{tabular}{|c|c|c|c|c|c|c|}
\hline Domain & $\begin{array}{c}\text { Peptide } \\
\text { sequence }\end{array}$ & $\begin{array}{l}\text { Charge } \\
\text { state }\end{array}$ & $\begin{array}{c}\text { Theoretical } \\
\mathbf{m} / \mathbf{z}\end{array}$ & $\begin{array}{c}\text { Observed } \\
\mathbf{m} / \mathbf{z}\end{array}$ & $\begin{array}{c}\text { Mass } \\
\text { accuracy } \\
(\mathbf{p p m}) \\
\end{array}$ & Glycoform \\
\hline \multirow{17}{*}{$\mathrm{C} 1$} & \multirow{11}{*}{ IHLENVTE } & 2 & 996.9357 & 996.9332 & -2.5 & HexNAc2Hex3Fuc1 \\
\hline & & 2 & 923.9068 & 923.9056 & -1.3 & HexNAc2Hex3 \\
\hline & & 2 & 1004.9332 & 1004.9327 & -0.5 & HexNAc2Hex4 \\
\hline & & 2 & 1077.9621 & 1077.9573 & -4.5 & HexNAc2Hex4Fuc1 \\
\hline & & 2 & 1085.9596 & 1085.9603 & 0.6 & HexNAc2Hex5 \\
\hline & & 2 & 1166.9860 & 1166.9860 & 0.0 & HexNAc2Hex6 \\
\hline & & 2 & 1240.0149 & 1240.0160 & 0.9 & HexNAc2Hex6Fuc1 \\
\hline & & 2 & 1248.0124 & 1248.0112 & -1.0 & HexNAc2Hex7 \\
\hline & & 2 & 1321.0413 & 1321.0443 & 2.3 & HexNAc2Hex7Fuc1 \\
\hline & & 2 & 1329.0388 & 1329.0377 & -0.8 & HexNAc2Hex8 \\
\hline & & 2 & 1410.0652 & 1410.0675 & 1.6 & HexNAc2Hex9 \\
\hline & \multirow{6}{*}{$\begin{array}{l}\text { LTPLCVTL } \\
\text { NCTTLTK }\end{array}$} & 3 & 930.4325 & 930.4305 & -2.1 & HexNAc2Hex4 \\
\hline & & 3 & 984.4501 & 984.4518 & 1.7 & HexNAc2Hex5 \\
\hline & & 3 & 1038.4677 & 1038.4674 & -0.3 & HexNAc2Hex6 \\
\hline & & 3 & 1092.4853 & 1092.4846 & -0.6 & HexNAc2Hex7 \\
\hline & & 3 & 1146.5029 & 1146.5023 & -0.5 & HexNAc2Hex8 \\
\hline & & 3 & 1200.5205 & 1200.5197 & -0.7 & HexNAc2Hex9 \\
\hline \multirow{21}{*}{$\mathrm{V} 1 / \mathrm{V} 2$} & \multirow{7}{*}{ VNITNFSK } & 2 & 907.9119 & 907.9118 & -0.1 & HexNAc2Hex3 \\
\hline & & 2 & 988.9383 & 988.9380 & -0.3 & HexNAc2Hex4 \\
\hline & & 2 & 1069.9647 & 1069.9644 & -0.3 & HexNAc2Hex5 \\
\hline & & 2 & 1150.9911 & 1150.9907 & -0.3 & HexNAc2Hex6 \\
\hline & & 2 & 1232.0175 & 1232.0165 & -0.8 & HexNAc2Hex7 \\
\hline & & 2 & 1313.0439 & 1313.0432 & -0.5 & HexNAc2Hex8 \\
\hline & & 2 & 1394.0703 & 1394.0687 & -1.1 & HexNAc2Hex9 \\
\hline & \multirow{9}{*}{ IGNITDEVR } & 2 & 954.9308 & 954.9302 & -0.6 & HexNAc2Hex3 \\
\hline & & 2 & 1035.9572 & 1035.9565 & -0.7 & HexNAc2Hex4 \\
\hline & & 2 & 1116.9836 & 1116.9822 & -1.3 & HexNAc2Hex5 \\
\hline & & 2 & 1198.0100 & 1198.0103 & 0.3 & HexNAc2Hex6 \\
\hline & & 2 & 1279.0364 & 1279.0358 & -0.5 & HexNAc2Hex7 \\
\hline & & 2 & 1360.0628 & 1360.0621 & -0.5 & HexNAc2Hex8 \\
\hline & & 2 & 1441.0892 & 1441.0889 & -0.2 & HexNAc2Hex9 \\
\hline & & 2 & 1027.9598 & 1027.9601 & 0.3 & HexNAc2Hex3Fuc1 \\
\hline & & 2 & 1108.9862 & 1108.9956 & 8.5 & HexNAc2Hex4Fuc1 \\
\hline & \multirow{5}{*}{$\underline{\mathbf{N} C S F} \underset{\mathrm{E}}{\mathrm{N} M T T}$} & 2 & 998.3688 & 998.3655 & -3.3 & HexNAc2Hex3 \\
\hline & & 2 & 1071.3978 & 1071.3944 & -3.2 & HexNAc2Hex3Fuc1 \\
\hline & & 2 & 1079.3952 & 1079.3936 & -1.5 & HexNAc2Hex4 \\
\hline & & 2 & 1160.4216 & 1160.4214 & -0.2 & HexNAc2Hex5 \\
\hline & & 2 & 1241.4480 & 1241.4464 & -1.3 & HexNAc2Hex6 \\
\hline
\end{tabular}




\begin{tabular}{|c|c|c|c|c|c|c|}
\hline Domain & $\begin{array}{l}\text { Peptide } \\
\text { sequence }\end{array}$ & $\begin{array}{c}\text { Charge } \\
\text { state }\end{array}$ & $\begin{array}{c}\text { Theoretical } \\
\mathbf{m} / \mathbf{z}\end{array}$ & $\begin{array}{c}\text { Observed } \\
\mathbf{m} / \mathbf{z}\end{array}$ & $\begin{array}{c}\text { Mass } \\
\text { accuracy } \\
(\mathrm{ppm})\end{array}$ & Glycoform \\
\hline & & 2 & 1322.4744 & 1322.4731 & -1.0 & HexNAc2Hex7 \\
\hline & & 2 & 1403.5008 & 1403.5007 & -0.1 & HexNAc2Hex8 \\
\hline & & 2 & 1484.5272 & 1484.5217 & -3.7 & HexNAc2Hex9 \\
\hline & \multirow{5}{*}{$\begin{array}{l}\text { LDLVQME } \\
\text { GNTSEYR }\end{array}$} & 3 & 849.7019 & 849.7011 & -0.9 & HexNAc2Hex3 \\
\hline & & 3 & 903.7195 & 903.7184 & -1.2 & HexNAc2Hex4 \\
\hline & & 3 & 957.7371 & 957.7359 & -1.3 & HexNAc2Hex 5 \\
\hline & & 3 & 1011.7547 & 1011.7531 & -1.6 & HexNAc2Hex6 \\
\hline & & 3 & 1065.7723 & 1065.7703 & -1.9 & HexNAc2Hex7 \\
\hline \multirow{21}{*}{$\mathrm{C} 2$} & \multirow{7}{*}{ LINCN $\frac{\mathrm{K}_{\mathrm{K}}}{\mathrm{TSVI}}$} & 2 & 1027.4770 & 1027.4768 & -0.2 & HexNAc2Hex3 \\
\hline & & 2 & 1108.5034 & 1108.5029 & -0.5 & HexNAc2Hex4 \\
\hline & & 2 & 1189.5298 & 1189.5299 & 0.1 & HexNAc2Hex5 \\
\hline & & 2 & 1270.5562 & 1270.5558 & -0.3 & HexNAc2Hex6 \\
\hline & & 2 & 1351.5826 & 1351.5819 & -0.5 & HexNAc2Hex7 \\
\hline & & 2 & 1432.6090 & 1432.6077 & -0.9 & HexNAc2Hex 8 \\
\hline & & 2 & 1513.6354 & 1513.6331 & -1.5 & HexNAc2Hex9 \\
\hline & \multirow{7}{*}{$\begin{array}{c}\text { NFTGTGPC } \\
\mathrm{K}\end{array}$} & 2 & 937.3851 & 937.3829 & -2.3 & HexNAc2Hex3 \\
\hline & & 2 & 1018.4115 & 1018.4167 & 5.1 & HexNAc2Hex4 \\
\hline & & 2 & 1099.4379 & 1099.4371 & -0.7 & HexNAc2Hex5 \\
\hline & & 2 & 1180.4643 & 1180.4608 & -3.0 & HexNAc2Hex6 \\
\hline & & 2 & 1261.4907 & 1261.4904 & -0.2 & HexNAc2Hex7 \\
\hline & & 2 & 1342.5171 & 1342.5160 & -0.8 & HexNAc2Hex8 \\
\hline & & 2 & 1423.5435 & 1423.5417 & -1.3 & HexNAc2Hex9 \\
\hline & \multirow{7}{*}{$\begin{array}{l}\text { TIIVHLNK }( \\
\text { S) }\end{array}$} & 2 & 915.4537 & 915.4538 & 0.1 & HexNAc2Hex3 \\
\hline & & 2 & 996.4801 & 996.4771 & -3.0 & HexNAc2Hex 4 \\
\hline & & 2 & 1077.5065 & 1077.5059 & -0.6 & HexNAc2Hex 5 \\
\hline & & 2 & 1158.5329 & 1158.5338 & 0.8 & HexNAc2Hex6 \\
\hline & & 2 & 1239.5593 & 1239.5598 & 0.4 & HexNAc2Hex7 \\
\hline & & 2 & 1320.5857 & 1320.5844 & -1.0 & HexNAc2Hex8 \\
\hline & & 2 & 1401.6121 & 1401.6101 & -1.4 & HexNAc2Hex9 \\
\hline \multirow{8}{*}{$\mathrm{C} 2 / \mathrm{V} 3$} & \multirow{8}{*}{$\begin{array}{c}\text { SVEINCTRP } \\
\text { SNNTR }\end{array}$} & 3 & 1414.8999 & 1414.8997 & -0.1 & HexNAc4Hex11 \\
\hline & & 3 & 1468.9175 & 1468.9110 & -4.4 & HexNAc4Hex12 \\
\hline & & 3 & 1522.9351 & 1522.9393 & 2.8 & HexNAc4Hex13 \\
\hline & & 3 & 1576.9527 & 1576.9525 & -0.1 & HexNAc4Hex14 \\
\hline & & 3 & 1630.9703 & 1630.9675 & -1.7 & HexNAc4Hex15 \\
\hline & & 3 & 1684.9879 & 1684.9899 & 1.2 & HexNAc4Hex16 \\
\hline & & 3 & 1739.0055 & 1739.0073 & 1.0 & HexNAc4Hex17 \\
\hline & & 3 & 1793.0231 & 1793.0188 & -2.4 & HexNAc4Hex18 \\
\hline \multirow{6}{*}{$\mathrm{C} 3$} & \multirow{6}{*}{$\underset{\mathrm{K}}{\mathrm{AYCEINGT}}$} & 2 & 1047.4325 & 1047.4357 & 3.1 & HexNAc2Hex3Fuc1 \\
\hline & & 2 & 1055.4299 & 1055.4324 & 2.4 & HexNAc2Hex4 \\
\hline & & 2 & 1136.4563 & 1136.4597 & 3.0 & HexNAc2Hex5 \\
\hline & & 2 & 1217.4827 & 1217.4817 & -0.8 & HexNAc2Hex6 \\
\hline & & 2 & 1298.5091 & 1298.5087 & -0.3 & HexNAc2Hex7 \\
\hline & & 2 & 1379.5355 & 1379.5365 & 0.7 & HexNAc2Hex8 \\
\hline
\end{tabular}




\begin{tabular}{|c|c|c|c|c|c|c|}
\hline Domain & $\begin{array}{c}\text { Peptide } \\
\text { sequence }\end{array}$ & $\begin{array}{c}\text { Charge } \\
\text { state }\end{array}$ & $\begin{array}{c}\text { Theoretical } \\
\mathbf{m} / \mathbf{z}\end{array}$ & $\begin{array}{c}\text { Observed } \\
\mathbf{m} / \mathbf{z}\end{array}$ & $\begin{array}{c}\text { Mass } \\
\text { accuracy } \\
(\mathrm{ppm})\end{array}$ & Glycoform \\
\hline & & 2 & 1460.5619 & 1460.5604 & -1.0 & HexNAc2Hex9 \\
\hline & \multirow{6}{*}{$\begin{array}{c}\text { GEFFYCNT } \\
\text { TK }\end{array}$} & 2 & 1160.9616 & 1160.9607 & -0.8 & HexNAc2Hex4 \\
\hline \multirow{18}{*}{ V4 } & & 2 & 1241.9880 & 1241.9850 & -2.4 & HexNAc2Hex5 \\
\hline & & 2 & 1323.0144 & 1323.0131 & -1.0 & HexNAc2Hex6 \\
\hline & & 2 & 1404.0408 & 1404.0405 & -0.2 & HexNAc2Hex7 \\
\hline & & 2 & 1485.0672 & 1485.0654 & -1.2 & HexNAc2Hex8 \\
\hline & & 2 & 1566.0936 & 1566.0923 & -0.8 & HexNAc2Hex9 \\
\hline & \multirow{6}{*}{$\begin{array}{l}\text { LFNSTWIG } \\
\text { NGTMEGR }\end{array}$} & 3 & 1534.6047 & 1534.6080 & 2.2 & HexNAc4Hex13 \\
\hline & & 3 & 1588.6223 & 1588.6212 & -0.7 & HexNAc4Hex14 \\
\hline & & 3 & 1642.6399 & 1642.6362 & -2.3 & HexNAc4Hex15 \\
\hline & & 3 & 1696.6575 & 1696.6517 & -3.4 & HexNAc4Hex 16 \\
\hline & & 3 & 1750.6751 & 1750.6699 & -3.0 & HexNAc4Hex 17 \\
\hline & & 3 & 1804.6927 & 1804.7029 & 5.7 & HexNAc4Hex 18 \\
\hline & \multirow{7}{*}{$\begin{array}{c}\text { NSSDTPIVL } \\
\text { PCK }\end{array}$} & 2 & 1111.9958 & 1111.9948 & -0.9 & HexNAc2Hex3 \\
\hline & & 2 & 1193.0222 & 1193.0210 & -1.0 & HexNAc2Hex 4 \\
\hline & & 2 & 1274.0486 & 1274.0477 & -0.7 & HexNAc2Hex 5 \\
\hline & & 2 & 1355.0750 & 1355.0739 & -0.8 & HexNAc2Hex6 \\
\hline & & 2 & 1436.1014 & 1436.1008 & -0.4 & HexNAc2Hex7 \\
\hline & & 2 & 1517.1278 & 1517.1252 & -1.7 & HexNAc2Hex8 \\
\hline & & 2 & 1598.1542 & 1598.1537 & -0.3 & HexNAc2Hex9 \\
\hline \multirow{7}{*}{$\mathrm{C} 4$} & \multirow{7}{*}{$\begin{array}{l}\text { INCVSNITG } \\
\text { ILLTR }\end{array}$} & 3 & 822.7349 & 822.7348 & -0.1 & HexNAc2Hex3 \\
\hline & & 3 & 876.7525 & 876.7523 & -0.2 & HexNAc2Hex4 \\
\hline & & 3 & 930.7701 & 930.7678 & -2.5 & HexNAc2Hex5 \\
\hline & & 3 & 984.7877 & 984.7878 & 0.1 & HexNAc2Hex6 \\
\hline & & 3 & 1038.8053 & 1038.8047 & -0.6 & HexNAc2Hex7 \\
\hline & & 3 & 1092.8229 & 1092.8220 & -0.8 & HexNAc2Hex8 \\
\hline & & 3 & 1146.8405 & 1146.8389 & -1.4 & HexNAc2Hex9 \\
\hline \multirow{13}{*}{ V5 } & \multirow{13}{*}{$\begin{array}{c}\text { DGGDNNST } \\
\text { TNETFRPG } \\
\text { GGNIK }\end{array}$} & 3 & 1069.1181 & 1069.1160 & -2.0 & HexNAc2Hex4 \\
\hline & & 3 & 1123.1357 & 1123.1336 & -1.9 & HexNAc2Hex 5 \\
\hline & & 3 & 1177.1533 & 1177.1506 & -2.3 & HexNAc2Hex6 \\
\hline & & 3 & 1231.1709 & 1231.1682 & -2.2 & HexNAc2Hex7 \\
\hline & & 3 & 1285.1885 & 1285.1859 & -2.0 & HexNAc2Hex 8 \\
\hline & & 3 & 1339.2061 & 1339.2024 & -2.8 & HexNAc2Hex9 \\
\hline & & 4 & 1227.7357 & 1227.7415 & 4.7 & HexNAc4Hex 12 \\
\hline & & 4 & 1268.2489 & 1268.2557 & 5.4 & HexNAc4Hex 13 \\
\hline & & 4 & 1308.7621 & 1308.7639 & 1.4 & HexNAc4Hex14 \\
\hline & & 4 & 1349.2753 & 1349.2837 & 6.2 & HexNAc4Hex 15 \\
\hline & & 4 & 1389.7885 & 1389.7837 & -3.5 & HexNAc4Hex16 \\
\hline & & 4 & 1430.3017 & 1430.2964 & -3.7 & HexNAc4Hex 17 \\
\hline & & 4 & 1470.8149 & 1470.8050 & -6.7 & HexNAc4Hex 18 \\
\hline
\end{tabular}


Zhao et al. Table S5

Table S5. Sequence similarity of different clade B and clade C gp120s from previous studies compared to those from this study.

\begin{tabular}{|c|c|c|c|c|c|c|}
\hline & \multicolumn{2}{|c|}{ Clade B } & \multicolumn{2}{c|}{ Clade C } & \\
\hline$\%$ & JR-FL & B.700010040.C9 & C.97ZA012 & C.1086 & C.Con & Con-S \\
\hline Ba-L & 85.7 & 79.3 & 68.9 & 70.5 & 75.7 & 79.1 \\
\hline 96ZM651 & 68.9 & 67 & 75.6 & 74.7 & 81.1 & 77.1 \\
\hline IIIB & - & - & - & 71.1 & - & - \\
\hline
\end{tabular}


Table S6. Comparisons of identified glycoforms from different clade B and clade C gp120s expressed in 293 or $293 \mathrm{~T}$ cells.

\begin{tabular}{|c|c|c|c|c|c|c|}
\hline \multirow{4}{*}{ Domain } & \multicolumn{6}{|c|}{ 293T cell/ Clade B } \\
\hline & \multicolumn{3}{|c|}{ JR-FL'1 } & \multicolumn{3}{|c|}{ B.700010040.C9² } \\
\hline & \multirow{2}{*}{$\begin{array}{l}\text { Glycosylation } \\
\text { sites }\end{array}$} & \multicolumn{2}{|c|}{$\begin{array}{c}\text { Number of } \\
\text { glycoforms } \\
\text { (common, unique) }\end{array}$} & \multirow{2}{*}{$\begin{array}{l}\text { Glycosylation } \\
\text { sites }\end{array}$} & \multicolumn{2}{|c|}{$\begin{array}{c}\text { Number of } \\
\text { glycoforms } \\
\text { (common, unique) }^{\mathrm{a}}\end{array}$} \\
\hline & & $\begin{array}{c}\text { vs } \\
\text { Ba-L }\end{array}$ & $\begin{array}{c}\text { vs } \\
96 Z M 651\end{array}$ & & $\begin{array}{c}\text { vs } \\
\text { Ba-L }\end{array}$ & $\begin{array}{c}\text { vs } \\
96 Z M 651\end{array}$ \\
\hline \multirow[b]{2}{*}{ C1 } & \multicolumn{3}{|c|}{ missing } & \multicolumn{3}{|c|}{ missing } \\
\hline & $\begin{array}{c}\text { AYDTEVHNVW } \\
\text { ATHACVPTDPN } \\
\text { PQEVVLENVTE } \\
\text { HFNMWK }\end{array}$ & $\begin{array}{c}15 \\
(10,5)\end{array}$ & $\begin{array}{c}15 \\
(13,2)\end{array}$ & $\begin{array}{c}\text { AYDTEAHNVW } \\
\text { ATHACVPTDPN } \\
\text { PQEVELKNVTE } \\
\text { NFNMWENNMV } \\
\text { EQMHEDIISLW } \\
\text { DQSLKPCVK / } \\
\text { NVTENFNMWE } \\
\text { NNMVEQMHED } \\
\text { IISLWDQSLKP } \\
\text { CVK }\end{array}$ & $\begin{array}{c}28 \\
(8,20)\end{array}$ & $\begin{array}{c}28 \\
(16,12)\end{array}$ \\
\hline C1/V1 & \multirow{2}{*}{$\begin{array}{c}\text { LTPLCVTLNCK } \\
\text { DVNATNTTNDS } \\
\text { EGTMER }\end{array}$} & \multirow[b]{2}{*}{$44^{\mathrm{b}}$} & \multirow[b]{2}{*}{$44^{\mathrm{b}}$} & \multirow{2}{*}{$\begin{array}{c}\text { LTPLCVTLNCT } \\
\text { DLGNVTNTTNS } \\
\text { NGEMMEK }\end{array}$} & \multirow{2}{*}{\multicolumn{2}{|c|}{ not provided ${ }^{c}$}} \\
\hline \multirow{3}{*}{ V1/V2 } & & & & & & \\
\hline & $\begin{array}{c}\text { GEIKNCSFNITT } \\
\text { SIRDEVQKI } \\
\text { NCSFNITTSIRD } \\
\text { EVQK }\end{array}$ & $\begin{array}{c}38 \\
(8,30)\end{array}$ & $\begin{array}{c}38 \\
(17,21)\end{array}$ & NCSFK & $\begin{array}{c}16 \\
(9,7)\end{array}$ & $\begin{array}{c}16 \\
(10,6)\end{array}$ \\
\hline & $\begin{array}{l}\text { LDVVPIDNNNT } \\
\text { SYR }\end{array}$ & 21 & $\begin{array}{c}21 \\
(15,6)\end{array}$ & LDVVPINDTR & 58 & $\begin{array}{c}58 \\
(33,25)\end{array}$ \\
\hline \multirow{6}{*}{$\mathrm{C} 2$} & \multicolumn{3}{|c|}{ missing } & $\begin{array}{c}\text { LVSCNTSVITQ } \\
\text { ACPK }\end{array}$ & $\begin{array}{c}48 \\
(37,11)\end{array}$ & $\begin{array}{c}48 \\
(35,13)\end{array}$ \\
\hline & \multicolumn{3}{|c|}{ missing } & \multicolumn{3}{|c|}{ missing } \\
\hline & \multicolumn{3}{|c|}{ missing } & \multicolumn{3}{|c|}{ missing } \\
\hline & $\begin{array}{l}\text { NVSTVQCTHGI } \\
\text { RPVVSTQLLLN } \\
\text { GSLAEEEVVIR }\end{array}$ & $30^{\mathrm{b}}$ & $30^{b}$ & $\begin{array}{l}\text { QFIGTGPCTNV } \\
\text { STVQCTHGIRP } \\
\text { VVSTQLLLNGS } \\
\text { LAEEEVVIR }\end{array}$ & $8^{b}$ & $8^{b}$ \\
\hline & $\begin{array}{l}\text { SDNFTNNAK / } \\
\text { SDNFTNNAKTII } \\
\text { VQLK }\end{array}$ & 17 & $\begin{array}{c}17 \\
(11,6)\end{array}$ & SVNFSDNAK & 25 & $\begin{array}{c}25 \\
(9,16)\end{array}$ \\
\hline & \multicolumn{2}{|l|}{ missing } & & TIIVQLNK & $10(7,3)$ & $10(7,3)$ \\
\hline
\end{tabular}




\begin{tabular}{|c|c|c|c|c|c|c|}
\hline \multirow{4}{*}{ Domain } & \multicolumn{6}{|c|}{ 293T cell/ Clade B } \\
\hline & \multicolumn{3}{|c|}{ JR-FL'1 } & \multicolumn{3}{|c|}{ B.700010040.C9² } \\
\hline & \multirow{2}{*}{$\begin{array}{l}\text { Glycosylation } \\
\text { sites }\end{array}$} & \multicolumn{2}{|c|}{$\begin{array}{c}\text { Number of } \\
\text { glycoforms } \\
\text { (common, unique) }^{\mathrm{a}}\end{array}$} & \multirow{2}{*}{$\begin{array}{l}\text { Glycosylation } \\
\text { sites }\end{array}$} & \multicolumn{2}{|c|}{$\begin{array}{c}\text { Number of } \\
\text { glycoforms } \\
\text { (common, unique) }\end{array}$} \\
\hline & & $\begin{array}{l}\text { vs } \\
\text { Ba-L }\end{array}$ & $\begin{array}{c}\text { vs } \\
96 Z M 651\end{array}$ & & $\begin{array}{l}\text { vs } \\
\text { Ba-L }\end{array}$ & $\begin{array}{c}\text { vs } \\
96 Z M 651\end{array}$ \\
\hline C2/V3 & $\begin{array}{c}\text { ESVEINCTRPN } \\
\text { NNTRK } \\
\text { ESVEINCTRPN } \\
\text { NNTRR }\end{array}$ & 42 & 42 & $\begin{array}{c}\text { SVEITCTRPNN } \\
\text { NTR }\end{array}$ & 63 & $\begin{array}{c}63 \\
(37,26)\end{array}$ \\
\hline \multirow{4}{*}{ C3 } & QAHCNISR & $\begin{array}{c}32 \\
(10,22)\end{array}$ & $\begin{array}{c}32 \\
(8,24)\end{array}$ & $\begin{array}{c}\text { AYCEINGTEWH } \\
\text { STLK }\end{array}$ & $\begin{array}{c}57 \\
(16,41)\end{array}$ & $\begin{array}{c}57 \\
(7,50)\end{array}$ \\
\hline & AKWNDTLK & $\begin{array}{c}28 \\
(26,2)\end{array}$ & $\begin{array}{c}28 \\
(4,24)\end{array}$ & \multicolumn{3}{|c|}{ missing } \\
\hline & LREQFENK & $\begin{array}{c}31 \\
(21,10) \\
\end{array}$ & 31 & $\begin{array}{c}\text { EQYNK } \\
\text { LREQYNKK }\end{array}$ & $\begin{array}{c}29 \\
(15,14)\end{array}$ & 29 \\
\hline & \multicolumn{3}{|c|}{ missing } & TIVFNR & 11 & $11(5,6)$ \\
\hline \multirow[t]{3}{*}{ V4 } & \multirow[t]{2}{*}{$\begin{array}{c}\text { TIVFNHSGGDP } \\
\text { EIVMHSFNCGG } \\
\text { EFFYCNSTQLF } \\
\text { NSTWNNNTEG } \\
\text { SNNTEGNTITL } \\
\text { PCR }\end{array}$} & \multirow[t]{2}{*}{$10^{\mathrm{b}}$} & \multirow[t]{2}{*}{$10^{b}$} & $\begin{array}{c}\text { SSGGDPEIVMY } \\
\text { SFNCGGEFFY } \\
\text { CNSTK / } \\
\text { LFNSTWPWND } \\
\text { TK }\end{array}$ & $9^{b}$ & 9 \\
\hline & & & & $\begin{array}{l}\text { GSHDTNGTLIL } \\
\text { PCK }\end{array}$ & $\begin{array}{c}75 \\
(27,48) \\
\end{array}$ & 75 \\
\hline & & ssing & & & issing & \\
\hline C4 & CSSNITGLLLTR & $\begin{array}{c}26 \\
(11,15)\end{array}$ & 26 & CSSNITGLLLTR & $\begin{array}{c}8 \\
(7,1)\end{array}$ & 8 \\
\hline V5 & $\begin{array}{l}\text { DGGINENGTEI } \\
\text { FRPGGGDMR }\end{array}$ & $\begin{array}{c}54 \\
(8,46)\end{array}$ & $\begin{array}{c}54 \\
(12,42)\end{array}$ & $\begin{array}{c}\text { DGGYESNETD } \\
\text { EIFRPGGGDM } \\
\mathrm{R}\end{array}$ & $\begin{array}{c}49 \\
(9,40)\end{array}$ & $\begin{array}{c}49 \\
(12,37)\end{array}$ \\
\hline
\end{tabular}




\begin{tabular}{|c|c|c|c|c|c|c|}
\hline \multirow{4}{*}{ Domain } & \multicolumn{6}{|c|}{ 293T cell/ Clade C } \\
\hline & \multicolumn{3}{|c|}{ C. $97 Z A 012^{3}$} & \multicolumn{3}{|c|}{ C. $1086^{2,4}$} \\
\hline & \multirow{2}{*}{$\begin{array}{l}\text { Glycosylation } \\
\text { sites }\end{array}$} & \multicolumn{2}{|c|}{$\begin{array}{c}\text { Number of } \\
\text { glycoforms } \\
\text { (common, unique) }^{a}\end{array}$} & \multirow{2}{*}{$\begin{array}{l}\text { Glycosylation } \\
\text { sites }\end{array}$} & \multicolumn{2}{|c|}{$\begin{array}{c}\text { Number of } \\
\text { glycoforms } \\
\text { (common, unique) }^{a}\end{array}$} \\
\hline & & $\begin{array}{c}\text { vs } \\
\text { Ba-L }\end{array}$ & $\begin{array}{c}\text { vs } \\
\text { 96ZM651 }\end{array}$ & & $\begin{array}{l}\text { vs } \\
\text { Ba-L }\end{array}$ & $\begin{array}{c}\text { vs } \\
\text { 96ZM651 }\end{array}$ \\
\hline \multirow[b]{2}{*}{ C1 } & \multicolumn{3}{|c|}{ missing } & \multicolumn{3}{|c|}{ missing } \\
\hline & $\begin{array}{c}\text { EVHNVWATHA } \\
\text { CVPTDPNPQEI } \\
\text { VLENVTENFNM } \\
\text { WK }\end{array}$ & $\begin{array}{c}31 \\
(11,20)\end{array}$ & $\begin{array}{c}31 \\
(24,7)\end{array}$ & $\begin{array}{c}\text { EVHNVWATHA } \\
\text { CVPTDPNPQE } \\
\text { MVLANVTENFN } \\
\text { MWK / } \\
\text { EVHNVWATHA } \\
\text { CVPTDPNPQE } \\
\text { MVLANVTENFN } \\
\text { MWKNDMVEQ } \\
\text { MHEDIISLWDE } \\
\text { SLKPCVK }\end{array}$ & $\begin{array}{c}55 \\
(16,39)\end{array}$ & $\begin{array}{c}55 \\
(37,18)\end{array}$ \\
\hline C1/V1 & $\begin{array}{c}\text { LTPLCVTLHCT } \\
\text { NATFK }\end{array}$ & $\begin{array}{c}26 \\
(20,6)\end{array}$ & $\begin{array}{c}26 \\
(25,1)\end{array}$ & $\begin{array}{c}\text { LTPLCVTLNCT } \\
\text { NVK }\end{array}$ & $\begin{array}{c}35 \\
(26,9)\end{array}$ & $\begin{array}{c}35 \\
(31,4)\end{array}$ \\
\hline \multirow{3}{*}{ V1/V2 } & $\begin{array}{c}\text { NNVTNDMNKEI } \\
\mathrm{R}\end{array}$ & 15 & $\begin{array}{c}15 \\
(2,13)\end{array}$ & $\begin{array}{c}\text { GNESDTSEVM } \\
\mathrm{K}\end{array}$ & $33^{b}$ & $\begin{array}{c}33 \\
(2,31)\end{array}$ \\
\hline & NCSFNTTTEIR & $\begin{array}{c}45(14 \\
31)\end{array}$ & $\begin{array}{c}45(20, \\
25)\end{array}$ & NCSFK & $\begin{array}{c}14(11 \\
3)\end{array}$ & $14(12,2)$ \\
\hline & \multicolumn{3}{|c|}{ missing } & $\begin{array}{l}\text { LDVVPLNGNSS } \\
\text { SSGEYR }\end{array}$ & 41 & $\begin{array}{c}41 \\
(29,12) \\
\end{array}$ \\
\hline \multirow{6}{*}{ C2 } & $\begin{array}{c}\text { ENRNNSNNSE } \\
\text { YILINCNASTIT } \\
\text { QACPK }\end{array}$ & $29^{b}$ & $29^{b}$ & $\begin{array}{c}\text { LINCNTSAITQA } \\
\text { CPK }\end{array}$ & $\begin{array}{c}64 \\
(49,15)\end{array}$ & $\begin{array}{c}64 \\
(46,18)\end{array}$ \\
\hline & CNNK & 5 & 5 & CNNK & 8 & 8 \\
\hline & \multicolumn{3}{|c|}{ missing } & TFNGTGPCR & 11 & 11 \\
\hline & $\begin{array}{c}\text { GPCNNVSTVQ } \\
\text { CTHGIKPVVST } \\
\text { QLLLNGSLAEK/ } \\
\text { GPCNNVSTVQ } \\
\text { CTHGIKPVVST } \\
\text { QLLLNGSLAEK } \\
\text { EIIIR }\end{array}$ & $9^{b}$ & $9^{b}$ & $\begin{array}{c}\text { NVSTVQCTHGI } \\
\text { KPVVSTQLLLN } \\
\text { GSLAEEEIIIR }\end{array}$ & $\begin{array}{c}18 \\
(7,11)\end{array}$ & 18 \\
\hline & SENLTDNVK & 41 & $\begin{array}{c}41 \\
(26,15)\end{array}$ & SENLTNNAK & 20 & $\begin{array}{c}20 \\
(17,3)\end{array}$ \\
\hline & TIIVHLNK & $\begin{array}{c}31 \\
(7,24) \\
\end{array}$ & $\begin{array}{c}31 \\
(9,22) \\
\end{array}$ & & & \\
\hline C2/V3 & $\begin{array}{c}\text { SVEIVCTRPNN } \\
\text { NTR } \\
\text { SVEIVCTRPNN } \\
\text { NTRK }\end{array}$ & 42 & $\begin{array}{c}42 \\
(30,12)\end{array}$ & $\begin{array}{l}\text { TIIVHLNESVNI } \\
\text { VCTRPNNNTR }\end{array}$ & 48 & $\begin{array}{c}48 \\
(13,35)\end{array}$ \\
\hline C3 & QAYCNISGSK & $6(2,4)$ & $6(3,3)$ & QAHCNINESK & $9(2,7)$ & $9(3,6)$ \\
\hline
\end{tabular}




\begin{tabular}{|c|c|c|c|c|c|c|}
\hline \multirow{4}{*}{ Domain } & \multicolumn{6}{|c|}{ 293T cell/ Clade $C$} \\
\hline & \multicolumn{3}{|c|}{ C.97ZA012 3} & \multicolumn{3}{|c|}{ C. $1086^{2,4}$} \\
\hline & \multirow{2}{*}{$\begin{array}{l}\text { Glycosylation } \\
\text { sites }\end{array}$} & \multicolumn{2}{|c|}{$\begin{array}{c}\text { Number of } \\
\text { glycoforms } \\
\text { (common, unique) }^{a}\end{array}$} & \multirow{2}{*}{$\begin{array}{l}\text { Glycosylation } \\
\text { sites }\end{array}$} & \multicolumn{2}{|c|}{$\begin{array}{c}\text { Number of } \\
\text { glycoforms } \\
\text { (common, unique) }^{\mathrm{a}}\end{array}$} \\
\hline & & $\begin{array}{c}\text { vs } \\
\text { Ba-L }\end{array}$ & $\begin{array}{c}\text { vs } \\
96 Z M 651\end{array}$ & & $\begin{array}{c}\text { vs } \\
\text { Ba-L }\end{array}$ & $\begin{array}{c}\text { vs } \\
96 Z M 651\end{array}$ \\
\hline & $\begin{array}{l}\text { WNETLK } \\
\text { WNETLKR }\end{array}$ & $\begin{array}{c}32 \\
(18,14)\end{array}$ & $\begin{array}{c}32 \\
(4,28)\end{array}$ & WNNTLQK & $\begin{array}{c}23 \\
(15,8)\end{array}$ & $\begin{array}{c}23 \\
(4,19)\end{array}$ \\
\hline & LQENYNNNK & $5(3,2)$ & 5 & \multicolumn{3}{|c|}{ missing } \\
\hline & \multicolumn{3}{|c|}{ missing } & \multicolumn{3}{|c|}{ missing } \\
\hline \multirow{3}{*}{ V4 } & GEFFYCNTTR & $28^{b}$ & $28^{b}$ & $\begin{array}{c}\text { GEFFYCNTSDL } \\
\text { FNGTYR; } \\
\text { NGTYNHTGR }\end{array}$ & 53 & 53 \\
\hline & $\begin{array}{l}\text { LFNNNATEDET } \\
\text { ITLPCR }\end{array}$ & 34 & 34 & SSNGTITLQCK & $\begin{array}{c}53 \\
(30,23)\end{array}$ & 53 \\
\hline & $\begin{array}{l}\text { AMYAPPIAGNI } \\
\text { TCK }\end{array}$ & 38 & 38 & \multirow{2}{*}{$\begin{array}{l}\text { AIYAPPIEGEIT } \\
\text { CNSNITGLLLLR }\end{array}$} & \multirow{2}{*}{$8(7,1)$} & \multirow{2}{*}{8} \\
\hline C4 & SNITGLLLVR & $\begin{array}{c}32 \\
(15,17)\end{array}$ & 32 & & & \\
\hline V5 & $\begin{array}{l}\text { DGGEDNKTEEI } \\
\text { FRPGGGNMK }\end{array}$ & $\begin{array}{c}43 \\
(8,35)\end{array}$ & $\begin{array}{c}43 \\
(11,32)\end{array}$ & $\begin{array}{c}\text { DGGQSNETND } \\
\text { TETFRPGGGD } \\
\text { MR }\end{array}$ & $\begin{array}{c}37 \\
(8,29)\end{array}$ & $\begin{array}{c}37 \\
(12,25)\end{array}$ \\
\hline
\end{tabular}




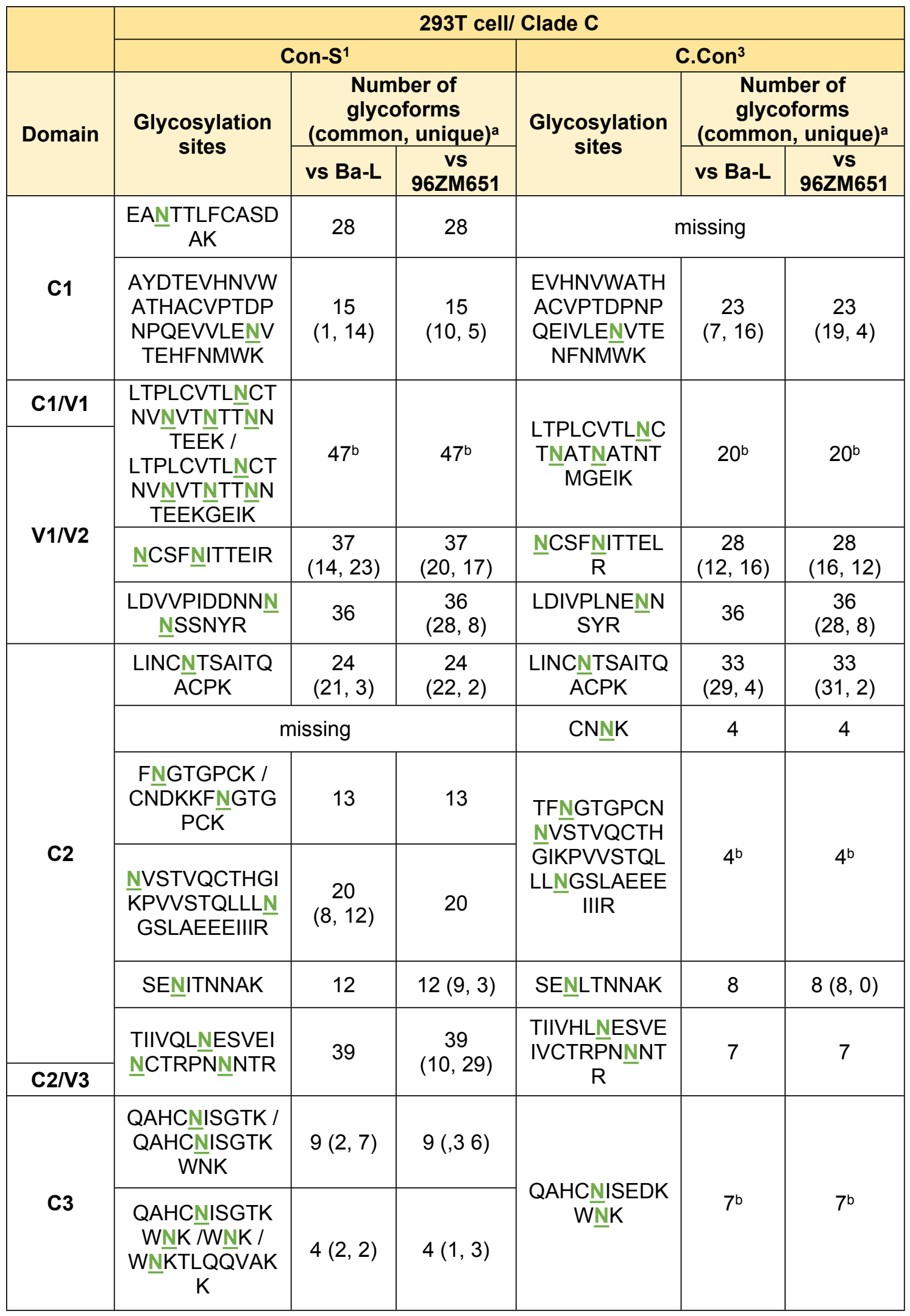




\begin{tabular}{|c|c|c|c|c|c|c|}
\hline \multirow{4}{*}{ Domain } & \multicolumn{6}{|c|}{ 293T cell/ Clade $C$} \\
\hline & \multicolumn{3}{|c|}{ Con-S1 } & \multicolumn{3}{|c|}{ C.Con ${ }^{3}$} \\
\hline & \multirow{2}{*}{$\begin{array}{l}\text { Glycosylation } \\
\text { sites }\end{array}$} & \multicolumn{2}{|c|}{$\begin{array}{c}\text { Number of } \\
\text { glycoforms } \\
\text { (common, unique) }^{a}\end{array}$} & \multirow{2}{*}{$\begin{array}{l}\text { Glycosylation } \\
\text { sites }\end{array}$} & \multicolumn{2}{|c|}{$\begin{array}{c}\text { Number of } \\
\text { glycoforms } \\
\text { (common, unique) }^{\mathrm{a}}\end{array}$} \\
\hline & & vs Ba-L & $\begin{array}{c}\text { vs } \\
96 Z M 651\end{array}$ & & vs Ba-L & $\begin{array}{c}\text { vs } \\
\text { 96ZM651 }\end{array}$ \\
\hline & $\begin{array}{l}\text { EHFNNK / } \\
\text { LREHFNNK }\end{array}$ & $\begin{array}{c}38 \\
(22,16)\end{array}$ & $\begin{array}{c}38 \\
(8,30)\end{array}$ & $\begin{array}{l}\text { LKEHFPNK / } \\
\text { EHFPNKTIK / } \\
\text { LKEHFPNKTIK }\end{array}$ & $\begin{array}{c}19 \\
(16,3)\end{array}$ & $\begin{array}{c}19 \\
(2,17)\end{array}$ \\
\hline & & ssing & & & issing & \\
\hline \multirow{3}{*}{ V4 } & $\begin{array}{l}\text { GEFFYCNTSGL } \\
\text { FNSTWIGNGTK }\end{array}$ & 68 & 68 & GEFFYCNTSK & 9 & 9 \\
\hline & $\begin{array}{l}\text { NNNNTNDTITL } \\
\text { PCR }\end{array}$ & $\begin{array}{c}34 \\
(16,18)\end{array}$ & 34 & $\begin{array}{l}\text { LFNSTYNSTN } \\
\text { STITLPCR }\end{array}$ & 37 & 37 \\
\hline & \multicolumn{3}{|c|}{ missing } & $\begin{array}{l}\text { AMYAPPIAGNI } \\
\text { TCK }\end{array}$ & 13 & 13 \\
\hline $\mathrm{C} 4$ & SNITGLLLTR & $\begin{array}{c}24 \\
(9,15) \\
\end{array}$ & 24 & SNITGLLLTR & $\begin{array}{c}29 \\
(11,18) \\
\end{array}$ & 29 \\
\hline V5 & $\begin{array}{c}\text { DGGNNNTNET } \\
\text { EIFRPGGGDM } \\
\mathrm{R}\end{array}$ & $\begin{array}{c}52 \\
(4,48)\end{array}$ & $\begin{array}{l}52(10 \\
42)\end{array}$ & $\begin{array}{c}\text { NNTETFRPGG } \\
\text { GDMR } \\
\text { DGGKNNTETF } \\
\text { RPGGGDMR }\end{array}$ & $\begin{array}{c}43 \\
(4,39)\end{array}$ & $\begin{array}{c}43 \\
(12,31)\end{array}$ \\
\hline
\end{tabular}

a. The number of glycoforms that were identified in this study and in a published work. In the bracket, common indicates common structures in both studies while unique indicates unique glycoforms identified in a published work.

b. The common or unique glycoforms were not listed since no common structures were observed between this study and a published work.

c. not provided ${ }^{c}$ : The identified glycoforms were not listed in the previously published study. 
Table S7. Comparisons of identified glycoforms from different clade B and clade C gp120s expressed in 293 or $293 \mathrm{~T}$ cells.

\begin{tabular}{|c|c|c|c|}
\hline \multirow{4}{*}{ Domain } & \multicolumn{3}{|c|}{ CHO cell/ Clade C } \\
\hline & \multicolumn{3}{|l|}{ C.10864 } \\
\hline & \multirow{2}{*}{$\begin{array}{l}\text { Glycosylation } \\
\text { sites }\end{array}$} & \multicolumn{2}{|c|}{$\begin{array}{l}\text { Number of glycoforms } \\
\text { (common, unique) a }\end{array}$} \\
\hline & & $\begin{array}{l}\text { vs. } \\
\text { IIIB }\end{array}$ & $\begin{array}{l}\text { vs. } \\
\text { 96ZM651 }\end{array}$ \\
\hline C1 & $\begin{array}{l}\text { EVHNVWATHACVPTDPNPQEMVL } \\
\text { ANVTENFNMWK }\end{array}$ & $20(16,4)$ & $20(16,4)$ \\
\hline $\mathrm{C} 1 / \mathrm{V} 1$ & LTPLCVTLNCTNNVK & 26 & $26(25,1)$ \\
\hline \multirow{3}{*}{ V1/V2 } & GNESDTSEVMK & $27^{\mathrm{b}}$ & $27(0,27)$ \\
\hline & NCSFK & $10(4,6)$ & $10(6,4)$ \\
\hline & LDVVPLNGNSSSSGEYR & 30 & $30(23,7)$ \\
\hline \multirow{6}{*}{ C2 } & LINCNTSAITQACPK & 43 & $43(31,12)$ \\
\hline & CNNK & 5 & 5 \\
\hline & TFNGTGPCR & \multicolumn{2}{|c|}{$11^{\mathrm{b}}$} \\
\hline & $\begin{array}{l}\text { NVSTVQCTHGIKPVVSTQLLLNGS } \\
\text { AFFFIIR }\end{array}$ & \multicolumn{2}{|c|}{$19^{b}$} \\
\hline & SENLTNNAK & $27(20,7)$ & $27(19,8)$ \\
\hline & \multirow[t]{2}{*}{ TIIVHLNESVNIVCTRPNNNNTR } & $30(7,23)$ & $30(7,23)$ \\
\hline $\mathrm{C} 2 / \mathrm{V} 3$ & & & \\
\hline \multirow{4}{*}{ C3 } & QAHCNINESK & $6(6,0)$ & $6(3,3)$ \\
\hline & WNNTLQK & $14(12,2)$ & $14(4,10)$ \\
\hline & \multicolumn{3}{|l|}{ missing } \\
\hline & \multicolumn{3}{|l|}{ missing } \\
\hline \multirow{2}{*}{ V4 } & $\begin{array}{c}\text { GEFFYCNTSDLFNGTYR/ } \\
\text { NGTYNHTGR }\end{array}$ & \multicolumn{2}{|c|}{$26^{\mathrm{b}}$} \\
\hline & SSNGTITLQCK & $37(22,15)$ & 37 \\
\hline C4 & AIYAPPIEGEITCNSNNITGLLLLR & $7(6,1)$ & 7 \\
\hline V5 & DGGQSNETNDTETFRPGGGDMR & $33(4,29)$ & $33(11,22)$ \\
\hline
\end{tabular}


a. The number of glycoforms that were identified in this study and in a published work. In the bracket, common indicates common structures in both studies while unique indicates unique glycoforms identified in a published work.

b. The common or unique glycoforms were not listed since no common structures were observed between this study and a published work. 


\section{Zhao et al. Figure S1}

\section{A. N88 site}

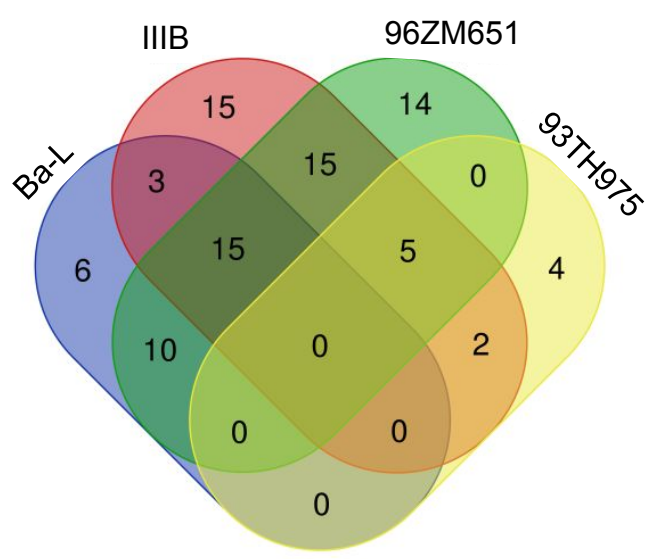

C. N332 site

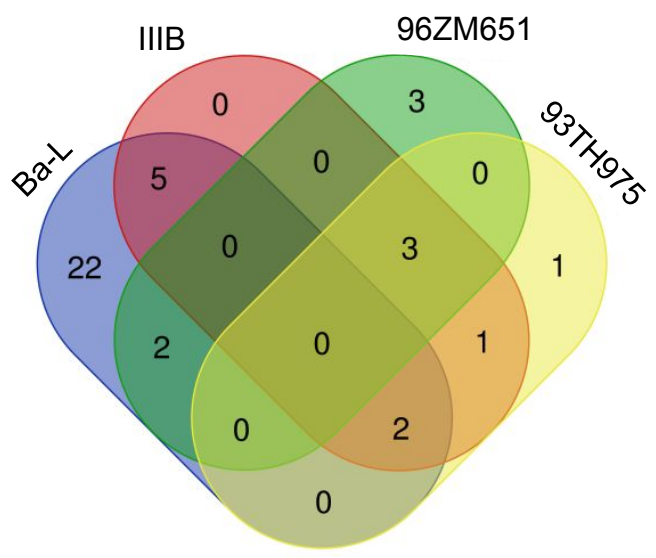

B. N156-N160 site

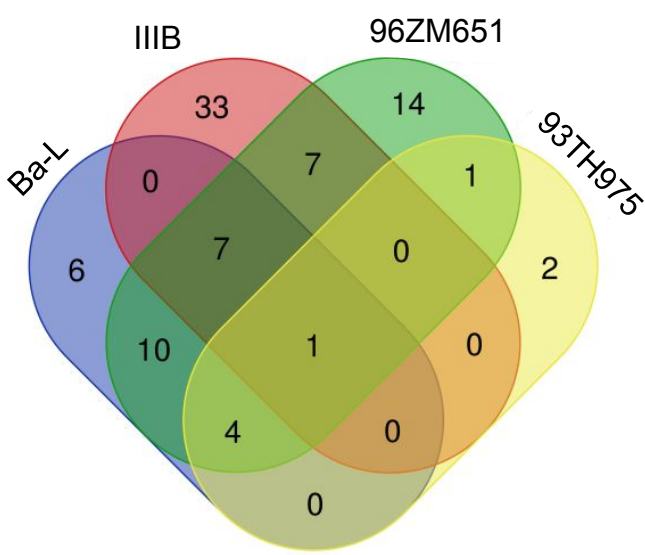

D. N234 site

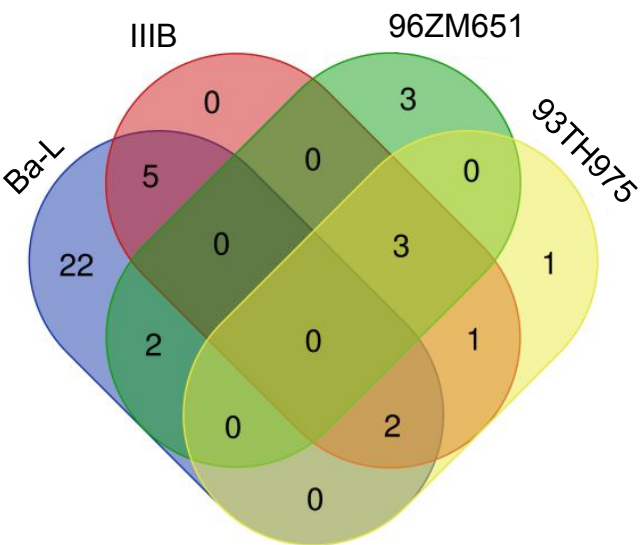

Figure S1. Venn plots of glycoforms detected at (A) N88, (B) N156-N160, (C) N332and (D) N234. On site N88, Ba-L and 96ZM651 shares 25 common structures. In which, 15 out of 25 structures were also detected in IIIB, while no glycans were detected in all 4 samples. On site N156-N160, the number of common structures detected in Ba-L, IIIB and 96ZM651 decreased to 8. Meanwhile 33 unique structures were identified from IIIB sample. Meanwhile, no common structure between the 4 samples were detected on site N332. Ba-L, in this case, has the most unique structures. On N234 site, all glycoforms detected from Ba-L and 93TH975 were also observed in the other 2 samples. On the contrary to N332, IIIB has the more unique glycans than the other 3 samples. 


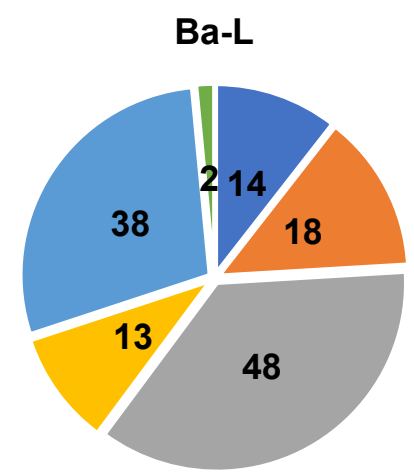

96ZM651

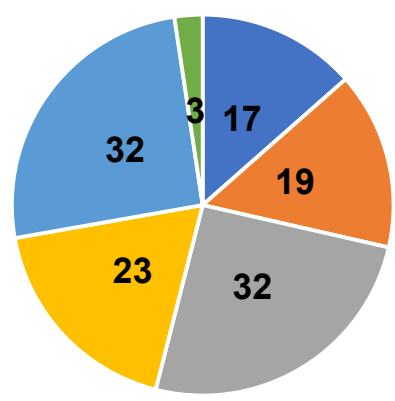

- High Mannose
IIIB

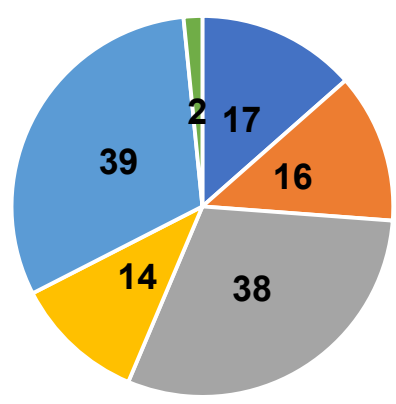

93TH975

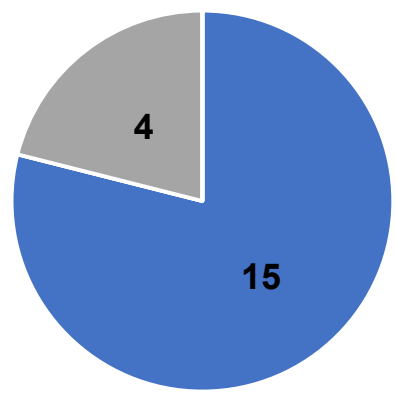

- Complex/Hybrid (not Fucosylated nor Sialylated)

- Fucosylated (not Sialylated)

- Sialylated (not Fucosylated)

- Fucosylated \& Sialylated

- Glycoforms Smaller Than the N-Glycan Core

Figure S2. Types of glycoforms identified from the samples. The glycans were classified into 6 categories: high mannose, complex/hybrid without fucose or sialic acid, fucosylated but not sialylated, sialylated but not fucosylated, fucosylated and sialylated and N-glycans smaller than the pentasaccharide core. 


\section{A. Total identified glycoforms}

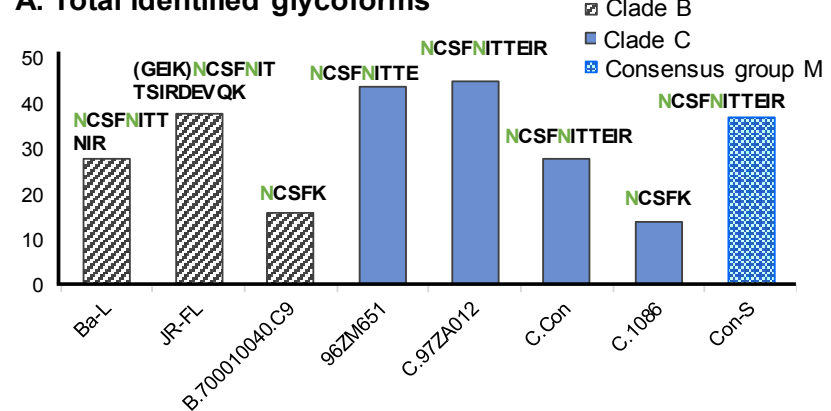

\section{B. Common glycoforms}

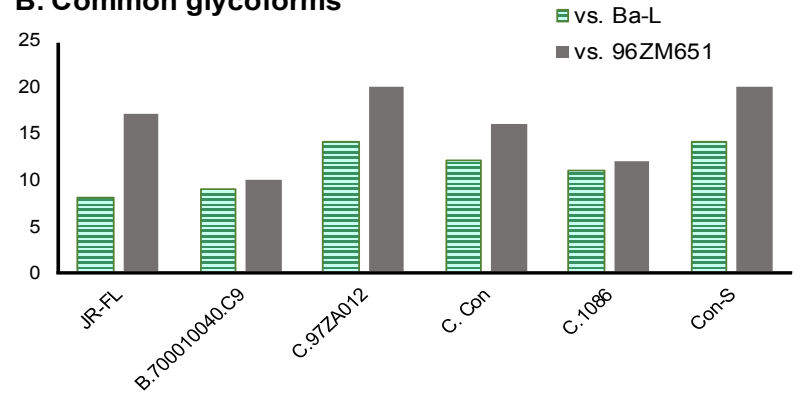

C. Unique glycoforms in previously published studies

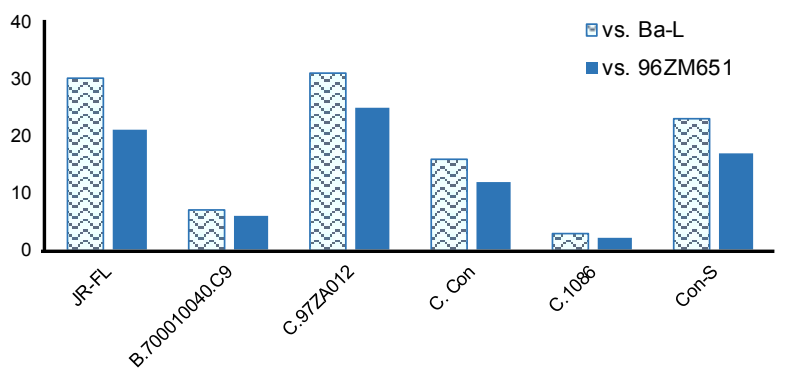

D. Unique glycoforms in this study compared to previously published studies

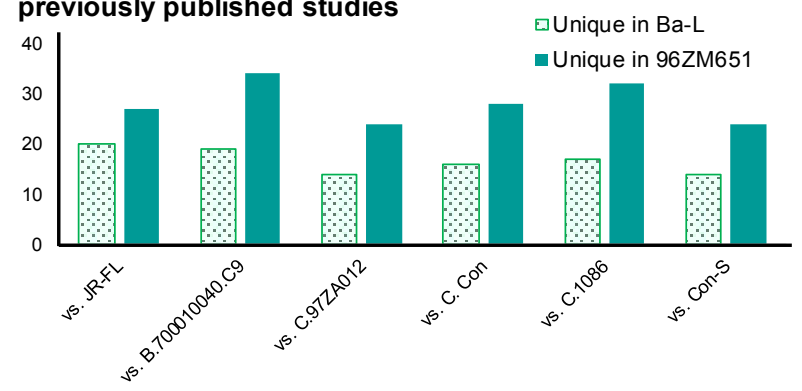

Figure S3. Direct comparisons of glycoforms at N156-N160 glycosylation sites with previously published studies. (A) The comparisons of total identified glycoforms. (B) Common glycoforms from the previous studies and this study. (C) unique glycoforms in previously published studies compared to this study. (D) Unique glycoforms from this study compared to previously published studies. 


\section{A. Total identified glycoforms}

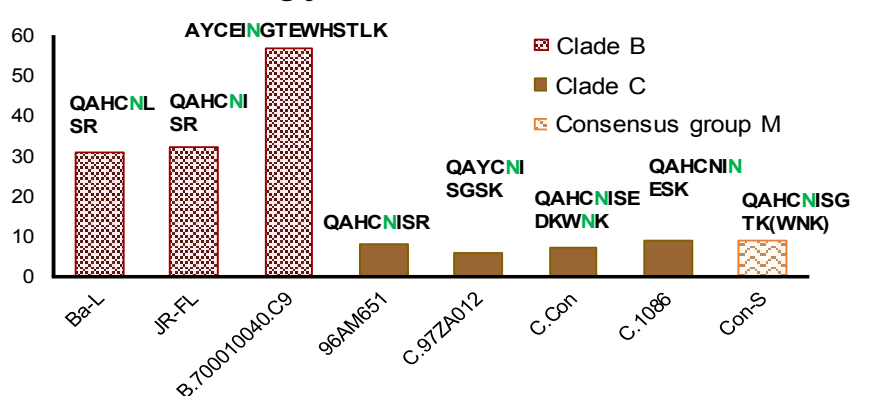

\section{B. Common glycoforms}

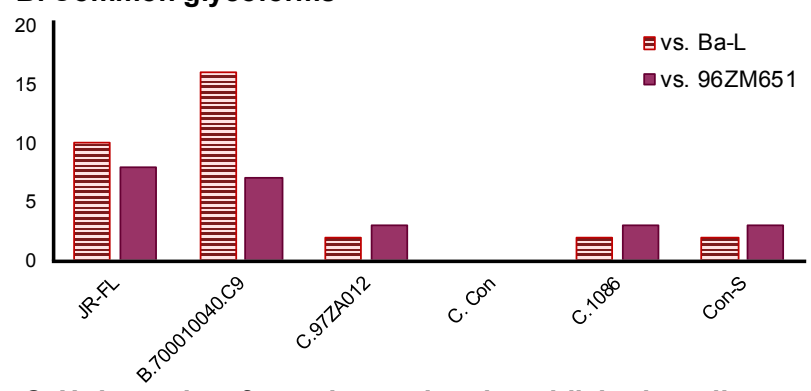

C. Unique glycoforms in previously published studies

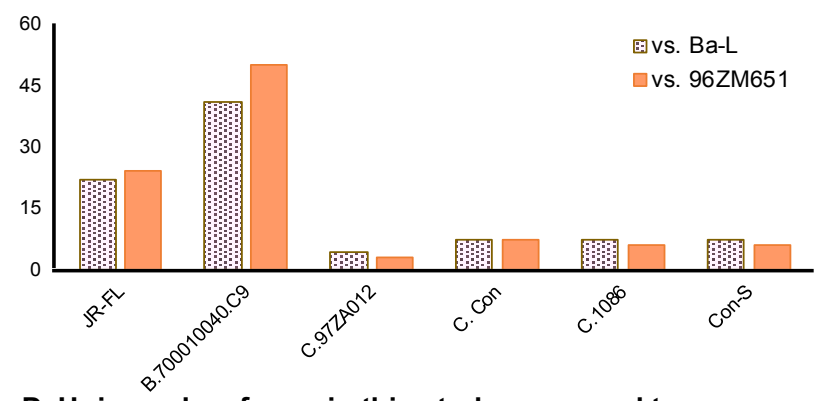

D. Unique glycoforms in this study compared to previously published studies $\quad$ uUnique in Ba-L

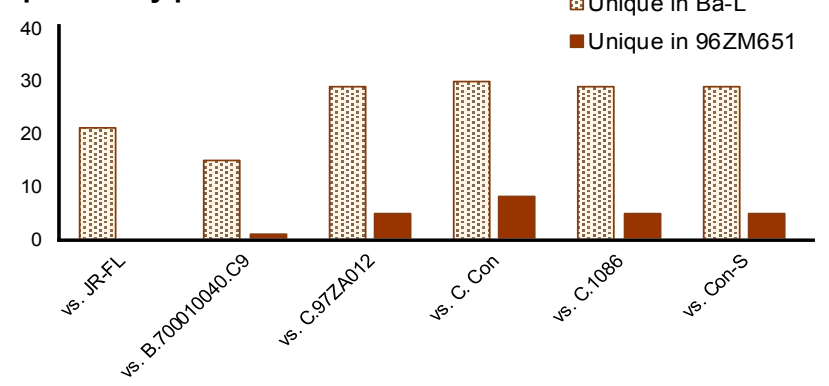

Figure S4. Direct comparisons of glycoforms at N332 glycosylation site with previously published studies. (A) The comparisons of total identified glycoforms. (B) Common glycoforms from the previous studies and this study. (C) unique glycoforms in previously published studies compared to this study. (D) Unique glycoforms from this study compared to previously published studies. 


\section{References}

(1) Go, E. P.; Irungu, J.; Zhang, Y.; Dalpathado, D. S.; Liao, H. X.; Sutherland, L. L.; Alam, S. M.; Haynes, B. F.; Desaire, H., Glycosylation site-specific analysis of HIV envelope proteins (JR-FL and CON-S) reveals major differences in glycosylation site occupancy, glycoform profiles, and antigenic epitopes' accessibility. J Proteome Res. 2008, 7, (4), 1660-74.

(2) Go, E. P.; Hewawasam, G.; Liao, H. X.; Chen, H.; Ping, L. H.; Anderson, J. A.; Hua, D. C.; Haynes, B. F.; Desaire, H., Characterization of glycosylation profiles of HIV-1 transmitted/founder envelopes by mass spectrometry. J Virol. 2011, 85, (16), 8270-84.

(3) Go, E. P.; Chang, Q.; Liao, H. X.; Sutherland, L. L.; Alam, S. M.; Haynes, B. F.; Desaire, H., Glycosylation site-specific analysis of clade C HIV-1 envelope proteins. J Proteome Res. 2009, 8, (9), 4231-42.

(4) Go, E. P.; Liao, H. X.; Alam, S. M.; Hua, D.; Haynes, B. F.; Desaire, H., Characterization of host-cell line specific glycosylation profiles of early transmitted/founder HIV-1 gp120 envelope proteins. J Proteome Res. 2013, 12, (3), 1223-34. 